\title{
Zur Theorie der Thetafunctionen von beliebig vielen Argumenten.
}

Von

M. Noether in Erlangen.

In dem vorliegenden Aufsatze soll die Darstellung des Additionstheorems der Thetafunctionen und der Thetarelationen, welche von Weber für die Functionen von 3 Argumenten ${ }^{*}$ ) und von mir (Math. Ann. XIV, pag. 248) für die von 4 Argumenten durchgeführt worden ist, auf die Thetafunctionen von beliebig vielen, $p$, Argumenten aus. gedehnt werden. Zu diesem $\mathrm{Zwecke}$ sind nicht eigentlich weitere neue Hilfsmittel in die Theorie einzuführen; vielmehr handelt es sich wesentlich nur darum, einzelne in dem letztgenannten Aufsatz nur vorübergehend zu Grunde gelegte Gesichtspunkte in ihrer vollen Be. deutung hervortreten zu lassen und die entsprechenden Hilfsmittel weiter auszubilden.

Zunächst ist die Untersuchung der Gruppirungsverhältnisse der Thetacharakteristiken (Abschnitt I.) die Grundlage der ganzen Entrvicklung; und hierzu dient der auch schon früher benutzte und als allgemein bezeichnete (Ann. XIV. p. 250) Ausgangspunkt: durch Betrachtung der zwei "Gruppen" gewisser Art gemeinsamen Charakteristiken die Theorie der $p$-reihigen (auf $\vartheta$-Functionen von $p$ Argumenten bezüglichen) Charakteristiken auf die der $(p-1)$-reihigen zurückzuführen. Dabei wird insbesondere von den Substitutionen, welche die Charakteristikenbeziehungen unverändert lassen (\$. 1.) - und welche zuerst von C. Jordan angegeben worden sind ${ }^{* *}$ ) -, in ausgedehnterem Masse Gebrauch gemacht. Auf diesem Wege tritt sogleich die Unterscheidung zwischen den $2^{2 p}$ eigentlichen und den $2^{2 p}-1$ Gruppen-Charakteristiken klar hervor; und indem bei den letzteren der Charakter der Geraden oder Ungeraden gar nicht in Betracht kommt, zeigt sich die Nothwendigkeit, die denselben zugeordneten Systeme aus geraden

*) H. Weber, Theorie der Abel'schen Funct. vom Geschlecht 3. Berlin 1876.

**) C. Jordan, Traité des substitutions, p. 229. 
Charakteristiken ganz ebenso zu behandeln, wie früher diejenigen aus ungeraden. Durch dieses gleichmässigere Verfabren werden jetzt die Beweise zu gleicher Zeit viel einfacher und durchsichtiger, und es gelingt jetzt, durch wenige Betrachtungen die Systeme von Gruppen (§ 2. $-\S 5$.) und sämmtliche Systeme von eigentlichen Charakteristiken (\$ 6. - \$. 10.) herzustellen. - Es würde sieh hieraus auch ohne Weiteres eine Groppentheorie der zugehörigen Substitutionen bilden lassen; indessen ist hier nur vorübergehend $(\$ 5 ., 3 . ; \S 11 ., 2 ., 6$.) auf diesen Punkt hingewiesen worden.

Eine allgemeine Theorie der Charakteristiken, wie sie im ersten Abschnitte gegeben ist, - nämlich eine solche, welche nur Eigenschaften benutzt, die bei den den Charakteristikenbeziehungen eigenen Substitutionen invariant sind - wird nothwendig, sobald man einen Ueberblick über solche Beziehungen gewinnen will. Die bisher nach Weierstrass oder Riemann angewandten Methoden zur Aufstellung ron Systemen für $p>3$ genügen dieser Forderung nicht, wie man schon daran erkennen kann, dass der wesentliche Unterschied zwischen eigentlichen und Gruppencharakteristiken, von denen die ersteren in zwei getrenrite Classen zerfallen, die anderen ein einziges System bildew, dabei nicht zum Ausdruck gekommen ist. Ausserdem bat die Methode von Weierstrass*) und Riemann**) eine weitere Theorie zur Grundlage: die Normirung der Wege für die hyperelliptischen Integrale und die Sätze über das Verschwinden der zugehörigen Thetafanctionen, und liefert zunächst nur ein bei Beiden identisches specielles System.

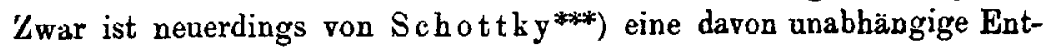
wicklung eines solchen speciellen Systens gegebeu worden, aber wiederum ohne Darstellung der charakteristischen Eigenschaften, welche den Uebergang zu den allgemeinen Systemen bewirken würden. Auch die von Prym $\dagger$ ) gegebene Ausdehnung des Riemann'schen Satzes auf alle Zerschneiduugen der 2-blättrigen Ebene in eine einfach zusammenhängende giebt zunächst nur die Existenz der Systeme, aber noch keine Uebersicht über deren Gestalt und Zusammenhang. Einige allgemeine Sätze über Gruppencharakteristiken hat neuerdings

*) Kön igsberger, Ueber die Transform. der A bel'echen Funct., Borch. J. 64.

*) Prym, Zur Theorie der Funct. in einer 2-blattrigen Ebene. Zürich 1866.

*) Scbottky, Abrise einer Theorie der Abel'schen Funct. ron 3 Var., 1. Theil. Breslau 1878.

†) Prym, a. c. $0 ., \S 12$. Unser Ausdruck "Gruppencharakteristik" ist nicht mit dem bei Prym vorkommenden zn verwechseln. Der letztere wäre identisch mit „einer Gruppencharakteristik zugeordneten halben Periode“, Perioden, die wie jene Charakteristiken nur eine Classe bilden; der eratere Begriff tritt bei Prym nicht a xplicite anf. Den eigentlichen Charakteristiken sind die zwei Classen von Thetafunctionen zugeordnet. 
Jordan (Compt. Rendus) mitgetheilt, wie es scheint mit ähnlichem Ausgangspunkt, wie der hier benutzte.

Un eine Anwendung der vorgetragenen allgemeinen Theorie zu geben, behandle ich im $\S 11$. die Methoden zur Auflösung derjenigen Gleichungen, deren Wurzeln den Charakteristiken oder Systemen von solchen zugeordnet werden können; insbesondere also die Gleichuugen, welche zur $\mathrm{Zweitheilung}$ der Perioden der Abel'schen Functionen dienen. Man kann diese Gleichungen lösen z. B. durch die successive Aufsuchung je einer Wurzel von Gleichungen der Grade

$$
2^{2} p-1, \quad 2^{2(p-1)}-1, \ldots, 2^{6}-1 \text {, }
$$

hierauf die vollständige Auflösung einer allgemeinen Gleichung rom $6^{\text {ten }}$ Grade und dann von $p^{2}-4$ quadratischen Gleichungen.

Aus jedem ,ausgezeichneterı" ( $\$ 8$.$) Charakteristikensystem kann$ man leicht Systeme von $2^{p}$ eigentlichen und von $2^{p}$ zugehörigen Gruppencharakteristiken bilden ( $\$ 13$.), deren Benutzung unmittelbar zu einem Ausdruck für das Additionstheorem der allgemeinen $\vartheta$-Functionen führt (\$14.)*). Aber die Coefficienten dieses Ausdrucks werden selbst wieder Summen von je $2^{p-3}$ Producten; und die Specialisirung der Variabeln in diesem Ausdruck liefert, sobald $p>4$ ist, nicht mehr die einfachsten Thetarelationen, welche überhaupt existiren.

Eine genauere Untersuchung, insbesondere der bemerkenswerthen, in lineare Factoren zerfallenden, Determinante der Gleichungen, ans welchen das Additionstheorem abgeleitet worden ist (\$ 15.), ergiebt nun, dass eine fundamentalere Formel als die genannte existirt, eine Formel, in welcher alle Coefficienten eingliedrig werden, während freilich die linke Seite selbst eine Summe von $2^{p-3}$ Ausdrücken mit zusammengesetzten Argumenten wird (\$ 16.). Diese Fundamentalformel der 'Theorie, welche auch für $p=4$ noch nicht in meinem oben genaunten Aufsatz (Avu. XIV.) enthalten ist, liefert sogleich die Thetarelationen ( $\S 17$.$) in Formen, die ebenso einfach sind, als für p=3$ (Weber) und für $p=4$; und die Theorie der Charakteristiken erlaubt ferner, auch die wichtige Frage nach den Beziehungen zwischen diesen Relationen wenigstens anzugreifeu und eine Reihe solcber fest-

*) Die Resultate bis $\$ 14$. incl. (excl. \$ 11.) finden sich kurz in meiner Note "Ueber die Thetacharaliteristiken", Sitz-ber. der Erl. Soc. v. 28. Juli 1879 (H. 11., p. 198) angegeben nod waren schon lăngere Zeit vorher in meinem Besitze. Dus Additionstheorem in der obigen Form, aus dem früher genannten speciellen System abgeleitet, ist seitdem auch von $\mathrm{H}$. Stahl (Borch J. 88, H. 2., ausgeg. den 14. Oct.d.J.) mitgetheilt worden, ohne Uebersicht über die Thetarelationen. Eine ebeu (zur Zeit des Druckes dieses Aufsatzes) erschieneue weitere Note von Stahl (Borch. J.) giebt eine unabhängige Definition des Riemann'schen Systems vou Gruppencharakteristiken, aber obne den Existenzbeweis und ohne Ontersuchung der Bildungsweise und Zusammenhïnge (Dez. 30.). 
zustellen ( $\left.\$ 18.)^{*}\right)$. - Zum Schlusse (\$ 19.) gebe ich eine Anwendung auf die Functionen für $p=5$, wobei ich insbesondere die Bedingungen discutire, unter welchen die $\vartheta$-Functionen zu hyperelliptischen werden.

\section{Abschnitt.}

Die Thetacharakteristiken.

\section{$\S 1$. \\ Definitionen nnd Operationen.}

1. In diesem ersten Abschuitte handelt es sich um eine Theorie der $2^{2 p} p$-reihigen Zahlencomplexe, welche aus

$$
(\alpha)=\left(\begin{array}{cccc}
n_{1}^{\alpha} & n_{2}^{\alpha} & \cdots & n_{p}^{\alpha} \\
m_{1}^{\alpha} & m_{z}^{\alpha} & \cdots & m_{p}^{\alpha}
\end{array}\right)
$$

entstehen, indem man allen Zahlen $n_{a}^{\alpha}$ und $m_{a}^{\alpha}$ unabhängig von einander die Werthe 0 oder 1 beilegt. Dieselben werden als eigentliche $p$-reihige Charaliteristiken, oder kürzer, wo keine Verwechslung entstehen kann, als "Charakteristiken" bezeichnet.

Insbesondere sei $n_{\mathfrak{a}}^{0}=m_{\mathfrak{a}}^{0}=0$, also

$$
(0)=\left(\begin{array}{llll}
0 & 0 & \cdots & 0 \\
0 & 0 & \cdots & 0
\end{array}\right) \text {. }
$$

Eine Charakteristik ( $\alpha$ ) wird gerade oder ungerade genannt, je nachdem

$$
\sum_{a=1}^{a=p} n_{a}^{\alpha} m_{a}^{\alpha}
$$

gerade oder ungerade ist. Dieser Ausdruck wird in Folgenden kürzer mit

bezeichnet; allgemeiner

$$
\sum n^{u} m^{u}
$$

$$
\sum_{a=1}^{a=p} n_{a}^{\alpha} m_{a}^{\beta}
$$

mit

$$
\sum n^{\alpha} m^{\beta}
$$

I. Es giebt $R_{p}=2^{p-1}\left(2^{p}-1\right)$ ungerade, $S_{p}=2^{p-1}\left(2^{p}+1\right)$ gerade Charakteristiken.

\#) Diese weiteren Resultate habe ich in einer Note in den Erl. Ber. rom 10. Nov. "Ueber die allgem. Thetafanctionen" kurz mitgetheilt. Der Inbalt von \$ 15. and \$ 16. ist von mir auch in der Sitz. der math. Section der Naturforscherversammlang in Baden (Sept. d. J.) gelegentlich erwähnt worden.

Mathematische Annalen. XVI. 
Denn sei hier, wie im Folgenden durchaus, gesetzt:

$$
R_{\mu}=2^{\mu-1}\left(2^{\mu}-1\right), \quad S_{\mu}=2^{\mu-1}\left(2^{\mu}+1\right) .
$$

Wir nehmen den Satz I., der für $p=1$ richtig ist, für die $(p-1)$ reihigren Charakteristiken als richtig an; ergänzt man sodann die $R_{p-1}$ ungeraden $(p-1)$-reihigen Charakteristiken durch eine der drei Reihen

$$
\begin{aligned}
& 0 \\
& 0
\end{aligned} \text { oder } \begin{aligned}
& 0 \\
& 1
\end{aligned} \text { oder } \begin{aligned}
& 1 \\
& 0
\end{aligned} \text {, }
$$

die $S_{p-1}$ geraden $(p-1)$-reihigen Charakteristiken durch die Reihe

za $p$-reihigen, so erhält man alle ungeraden Charakteristiken dieser Art, nämlich

$$
3 R_{p-1}+S_{p-1}=R_{p}
$$

und ebenso, durch Vertauschung dieser Ergänzungen, die

$$
3 S_{p-1}+R_{p-1}=S_{p}
$$

geraden Charakteristiken.

Als Summe $(\alpha \beta)$ zweier Charakteristiken

$$
(\alpha)=\left(\begin{array}{c}
n_{1}^{\alpha} \cdots n_{p}^{\alpha} \\
m_{1}^{\alpha} \cdots m_{p}^{\alpha}
\end{array}\right), \quad(\beta)=\left(\begin{array}{c}
n_{1}^{\beta} \cdots n_{p}^{r^{\beta}} \\
m_{1}^{\text {\% }} \cdots m_{p}^{\beta}
\end{array}\right)
$$

sei der Ausdruck bezeichnet:

wo

$$
(\alpha)+(\beta)=(\alpha \beta)=\left(\begin{array}{c}
n_{1}^{\alpha \beta} \cdots n_{p}^{\alpha_{\beta} \beta} \\
m_{1}^{\alpha \beta} \cdots m_{p}^{\alpha \beta}
\end{array}\right),
$$

$$
\left.\begin{array}{c}
n_{a}^{\alpha \beta} \equiv n_{a}^{\alpha}+n_{a}^{\beta} \\
m_{\alpha}^{\alpha \beta} \equiv m_{a}^{\alpha}+m_{a}^{\beta}
\end{array}\right\} \quad(\bmod , 2) .
$$

2. Wir ordnen nun alle diejenigen Paare von einander verschiedener ungerader Charakteristiken, sowie diejenigen Paare von einander versehiedener gerader Charakteristiken, welche dieselbe Summe $(r)$ geben, zu einer Gruppe zusanmen, und geben auch dieser Gruppe. eine entsprechende Charakteristik

$$
[r]
$$

die wir Gruppencharalteristik nennen. Sei

$$
(r)=(\alpha)+(\beta)=\left(\alpha^{\prime}\right)+\left(\beta^{\prime}\right)=\cdots,
$$

wo $(\alpha)$ und $(\beta)$ entweder beide gerade oder beide ungerade sind, ebenso $\left(\alpha^{\prime}\right),\left(\beta^{\prime}\right)$, etc., so sagen wir, dass $(\alpha),(\beta),\left(\alpha^{\prime}\right), \cdots$ in der Gruppe $[r]$ enthalten, und zwar $(\alpha),(\beta)$ gepaart enthalten sind. 
II. Es giebt $2^{2 p}-1$ Gruppencharaliteristiken.

Denn alle Zahlencomplexe

$$
[r]=\left[\begin{array}{cc}
n_{1}^{r} \cdots & n_{p}^{r} \\
m_{1}^{r} \cdots & m_{p}^{r}
\end{array}\right]
$$

treten als solche auf, ausgenommen [0].

3. $\mathrm{Zu}$ den beiden in 1 . and 2 . angegebenen Bedeutungen der Zahlencomplexe, $(\alpha)$ und $[r]$, fügen wir noch eine dritte, die einer Substitution. Seien nämlich wieder, wie in 2., die Lerlegungen der Gruppe $[r]$ :

$$
(\gamma)=(\alpha)+(\beta)=\left(\alpha^{\prime}\right)+\left(\beta^{\prime}\right)=\cdots ;
$$

so bezeichnen wir mit

$$
\{r\}
$$

diejenige Substitution, welche die in der Gruppe $[\bar{r} \bar{j}$ enthaltenen Charakteristiken in die mit ilmen darin gepaart auftretenden überführt, $d . b$. $(\alpha)$ mit $(\beta),\left(\alpha^{\prime}\right)$ mit $\left(\beta^{\prime}\right)$ etc. vertauscht, die nicht in $[r]$ enthaltenen Charakteristiken aber unveräudert lässt.

Um die weiteren Eigenschaften einer solchen Substitution $\{r\}$ zu ermitteln, betrachte man eine gerade Anzahl von Charakteristiken, in deren Summe alle Elemente zu 0 (mod. 2) werden, eine Sumne, die wir deshalb $=0$ setzen :

$$
\left(\alpha_{1}\right)+\left(\alpha_{2}\right)+\cdots+\left(\alpha_{2 \mu}\right)=0 .
$$

Von diesen Charakteristiken $\left(\alpha_{1}\right), \cdots\left(\alpha_{2 \mu}\right)$ seien $\nu$, nämlich

$$
\left(\alpha_{1}\right),\left(\alpha_{2}\right), \cdots\left(\alpha_{v}\right),
$$

gerade, die übrigen $2 \mu-v$ ungerade; also:

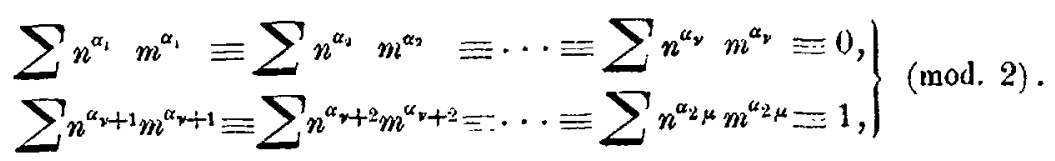

Ferner sei:

$$
\left.\begin{array}{l}
\sum n^{r \alpha_{i}} m^{r \alpha_{1}} \equiv \varepsilon_{1}, \quad \ldots, \sum n^{r \alpha_{v}} m^{r \mu_{v}} \equiv \varepsilon_{v}, \\
\sum n^{r \alpha_{v+1}} m^{r \alpha_{v+1}} \equiv \varepsilon_{v+1}+1, \cdots, \sum n^{r \alpha_{2 \mu} m^{r \alpha_{2 \mu}}} \equiv \varepsilon_{z \mu}+1,
\end{array}\right\} \text { (mod.2). }
$$

So oft nun eine Zahl $\varepsilon_{\varphi}=0$ ist, wird die entsprechende Charakteristik $\left(\alpha_{\rho}\right)$ durch die Substitution $\{r\}$ bez. in $\left(r \alpha_{p}\right)$ übergeführt, im andern Falle nicht. Addirt man aber die letzteren Congruenzen und benutzt die ersteren, so ergiebt sich: 
also:

$$
\begin{aligned}
& 2 \mu \sum n^{r} m^{r}+\sum n^{r} m^{\alpha_{1} \alpha_{2} \ldots \alpha_{2 \mu}}+\sum n^{\alpha_{1} \alpha_{2} \ldots \alpha_{2} \mu} m^{r}+ \\
& +\sum n^{\alpha_{1}} m^{\alpha_{1}}+\cdots+\sum n^{\alpha_{2 \mu}} m^{\alpha_{2 \mu}} \equiv 2 \mu-v \equiv \\
& \equiv \varepsilon_{1}+\varepsilon_{2}+\cdots+\varepsilon_{2 \mu}+2 \mu-\nu \text {; }
\end{aligned}
$$

$$
\varepsilon_{1}+\varepsilon_{2}+\cdots+\varepsilon_{2 \mu} \equiv 0 \quad(\bmod .2)
$$

d. h. in der Summe

$$
\left(\alpha_{1}\right)+\left(\alpha_{2}\right)+\cdots+\left(\alpha_{2 \mu}\right)=0
$$

wird nur eine gerade Anzahl der $\left(\alpha_{\varrho}\right)$ durch die Substitution $\{r\}$ je um $(r)$ vermehrt, so dass die ganze Summe nach der Substitution unverändert $=0$ bleibt.

Dasselbe findet natürlich auch statt, wenn man verschiedene Substitutionen der Form $\{r\}$ nach einander ausführt. Man erhält dadurch neue Substitutionen, welche unter den ursprünglichen der Form $\{r\}$ im Allgemeinen nicht enthalten sein werden.

Auf die Form (1) lässt sich jede Relation zwischen den Charakteristiken bringen; denn hätte man eine ungerade Anzahl

$$
\left(\alpha_{1}\right)+\left(\alpha_{2}\right)+\cdots+\left(\alpha_{2 \mu-1}\right)
$$

von Charakteristiken, in deren Summe alle Elemente congruent 0 werden, so setze man:

$$
\left(\alpha_{1}\right)+\left(\alpha_{2}\right)+\cdots+\left(\alpha_{2, u-1}\right)+(0)=0 \text {. }
$$

4. Aus 3. folgt, indem man eine oder zwei Charakteristiken $\left(\alpha_{e}\right)$ in der Beziehung (1) auf die rechte Seite bringt: dass man jedem System, das aus einer ungeraden Anzahl von Charaliteristiken besteht, als Zeichen eine der $2^{2 p}$ eigentlichen Charakteristiken zuordnen mag, nämlich dasjenige der Summe der Charakteristiken des Systems; und dass man jedem System, das aus einer geraden Anzahl von Charakteristiken besteht, als Zeichen eine der $2^{2 p}-1$ Gruppencharahteristiken zuordnen mag, ebenfalls das der Summe, wenn nämlich diese Summe nicht selbst schon $=0$ ist, was wir im Folgenden ausschliessen können.

Eine Substitution $\{r\}$ ändert dann im Allgemeinen sowohl System, als Zeichen des Systems, aber liefert immer wieder eine der Definition eutsprechende Zuordnung:

Wir bezeichnen ein solches System, und sein Zeichen in ersten Falle auf die Art:

$$
\left(\alpha_{1}\right) \equiv\left(\alpha_{2}\right),\left(\alpha_{3}\right), \cdots\left(\alpha_{2 \mu}\right),
$$

im zweiten Falle auf die Art:

$$
\left[\alpha_{1} \alpha_{2}\right] \equiv\left(\alpha_{3}\right),\left(\alpha_{4}\right), \cdots,\left(\alpha_{2 \mu}\right),
$$

wenn beide Male 


$$
\left(\alpha_{1}\right)+\left(\alpha_{z}\right)+\cdots+\left(\alpha_{z \mu}\right)=0
$$

sein sollte. Zum zweiten Falle grehören zum Beispiel die Zerlegungen vou Nr. 2. dieses $\S$, die hier so zu schreiben sind:

$$
[r] \equiv(\alpha),(\beta) \equiv\left(\alpha^{\prime}\right),\left(\beta^{*}\right) \equiv \cdots
$$

Die wesentliche Verschiedenheit in dem Verhalten der beiden betrachteten Arten von Systemen zejgt sich nun durch folgenden Satz:

III. Die aus einer ungeraden Anzahl von Charakteristiken bestehenden Systeme zerfallen in zwei völlig getrennte Classen, nämlich "je nachdem ihr Zeichen eive der $S_{p}$ geraden oder aber eine der $R_{p}$ ungeraden eigentlichen Charakteristiken ist. Die erste Classe bestelt aus $S_{p}$. Onterclassen, die einander in jeder Beziehuny vertreton können; die zweite Classe aus $R_{p}$ Unterclassen, die ebenfalls unter sich völlig gleichwerthig sind.

Die aus einer geraden Anzahl von Charalteristitien besteltenden Systeme zerfallen in $2^{2 p}-1$ Classen, von denen jede aus gleichviel Systemen, in gleicher Weise geordnet, besteht; den $2^{2 p}-1$ Gruppencharakteristiken entsprechend.

In der That ist es durch die Substitutionen nicht möglich, eine gerade eigentliche Charakteristik in eine ungerade überzuführen. Dagegen kann man irgend eine gerade Charakteristik $(\alpha)$ in die beliebige gerade Charakteristik $(\beta)$ durch die Substitution $\{\alpha \beta\}$ überführen; und ebenso, wenn $(\alpha)$ und $(\beta)$ ungerade sind. Alsdann gehen auch alle Systeme von $(\alpha)$ in ebenso geordnete von $(\beta)$ über. Die gerade Charakteristik $(0)$ ist dabei vor den übrigen in nichts ausgezeichnet. Der letzte Theil des Satzes 1II. wird sich aus folgendem Satze ergeben:

IV. Eine Gruppencharaliteristik $[r]$ wird durch eine Substitution $\{s\}$ in $[r s]$ übergefïhrt oder bleibt unveründert, je nachdem $K_{r, s} \equiv 1$ oder $=0(\bmod .2)$, wo

gesetzt ist.

$$
K_{r, d}=\sum\left(n^{r} m^{s}+n^{s} m^{r}\right)
$$

Denn man zerlege $[r]$ etwa in die Summe zweier geraden Charakteristiken:

so wird

$$
(r)=(\alpha)+(\beta),
$$

also:

$$
\left.\begin{array}{l}
\sum n^{\alpha} m^{\alpha} \equiv \sum n^{\beta} m^{\beta} \equiv 0, \\
\sum n^{s \alpha} m^{\alpha \alpha} \equiv \varepsilon, \quad \sum n^{s \beta} m^{z \beta} \equiv \varepsilon^{\prime},
\end{array}\right\}_{(\bmod .2)}
$$

$$
\sum\left(n^{r} m^{s}+n^{s} m^{r}\right) \equiv K_{r, s} \equiv \varepsilon+\varepsilon^{\prime} \quad(\bmod .2) \text {, }
$$

so dass durch $\{s\}$ für $K_{r,} \equiv 1$ eine der Grössen $(\alpha),(\beta)$, also $(r)$, 
um (s) vermehrt wird, für $K_{r, s} \equiv 0$ keine oder beide Grössen $(\alpha),(\beta)$, was der Satz IV. ist.

Es muss nun immer Substitutionen geben, welche irgend zwei Gruppencharakteristiken, $[r]$ und $\left[r^{\prime}\right]$, in einander überführen. Denn entweder hat man

$$
K_{r, r^{\prime}} \equiv 1 \quad(\bmod .2) ;
$$

dann leistet dieses nach IV. die Substitution $\left\{r r^{\prime}\right\}$, da

Oder es ist

$$
\left.K_{r, r r^{\prime}} \equiv K_{r, r}+K_{r, r^{\prime}} \equiv K_{r, r^{\prime}} \equiv 1 \quad \text { (mod. } 2\right) \text {. }
$$

$$
K_{r, r^{\prime}} \equiv 0 \quad(\bmod .2) \text {; }
$$

dann bestimme nan einen Zahlencomplex $(t)$ vou der Art, dass die Relationen

$$
\left.\begin{array}{l}
\mathcal{K}_{r, r}=\sum\left(n^{r} m^{r^{r}}+n^{r^{r}} m^{r}\right) \equiv 0 \\
K_{r, t}=\sum\left(n^{r} m^{t}+n^{t} m^{r}\right) \equiv 1 \\
\mathcal{K}_{r^{\prime}, t}=\sum\left(n^{r^{r}} m^{t}+n^{t} m^{r^{\prime}}\right) \equiv 1
\end{array}\right\} \text { (mod. 2) }
$$

zugleich erfüllt sind, was immer und auf mehrfache Art (s. unten § 2.) möglich ist; dann erreicht man die Ueberführung durch die beiden auf $[r]$ nach einander ausgeführten Substitutionen $\{r t\},\left\{r^{\prime} t\right\}$, da durch die erstere Substitution $[r]$ in $[t]$ übergeht, wegen $K_{r, r t}=1$, durch die zweite $[t]$ in $\left[r^{\prime}\right]$, wegen $K_{t, r^{\prime}} \equiv K_{r^{\prime}, t} \equiv 1$. Diese Ueberführung ist dann unmöglich, wenn eine der beiden Gruppencharakteristiken, $[r]$ oder $\left[r^{\prime}\right],=0$ wäre, und dieses bleibt daher ausgeschlossen. Die übrigen $2^{2 p}-1$ Gruppencharakteristiken, mit ihren Systemen, sind aber alle gleichwerthig, wie es der Satz III. aussagte.

\section{$\$ 2$.}

\section{Gruppenbeziehungen.}

Ueber die Gruppen und ihre Beziehungen existirt eine Reibe von Sätzen, die hier abgeleitet werden sollen.

V. Jede Gruppe $[r]$ besteht aus $R_{p-1}$ Paaren von je zwei ungeraden und aus $S_{p-1}$ Paaren von je zwei geraden Charaliteristiken.

Deun da sich die $R_{p}$ ungeraden Charakteristiken auf $\frac{1}{2} R_{p}\left(R_{p}-1\right)$ Weisen in Paare combiniren lassen und da jede der $2^{2} p-1$ Gruppen nach Satz III. gleichviele dieser Paare enthält, so besteht eine Gruppe $[r]$ aus

$$
\frac{\frac{1}{2} R_{p}\left(R_{p}-1\right)}{2^{2 p}-1}=R_{p-1}
$$

solcher Paare. Ebenso folgt der zweite Theil des Satzes.

VI. Zwei Gruppen [r], [s], welche in der Bezielung $K_{r, s} \equiv 1$ stehen, enthalten $R_{p-1}$ ungerade und $S_{p-1}$ gerade Charakteristiken ge. 
meinsam. Diese treten zugleich in lieiner der beiden Gruppen gepaart auf; d. h. jelles Pạar der Gruppe [r] enthält eine Charakteristik, welche zugleich in $[s]$ vorkommt. Drei Gruppen $[r],[s],[r s]$, fiir welche $K_{r, s} \equiv 1$, enthalten keine Charaliteristik gemeinsam.

Der erste Theil dieses Satzes ist nur eine andere Formulirung des ersten Theiles ron IV. Der letate Theil folgt unmittelbar daraus, indem man die Gruppe $[r s]$ durch Verbindung entsprechender Paare von $[r]$ und $[s]$ bildet, auf die Weise:

$$
\begin{aligned}
{[r] } & \equiv(\alpha),(\beta) \equiv\left(\alpha^{\prime}\right),\left(\dot{\beta}^{\prime}\right) \equiv \cdots, \\
{[s] } & \equiv(\alpha),(\gamma) \equiv\left(\alpha^{\prime}\right),\left(\gamma^{\prime}\right) \equiv \cdots, \\
{[r s] } & \equiv(\beta),(\gamma) \equiv\left(\beta^{\prime}\right),\left(\gamma^{\prime}\right) \equiv \cdots
\end{aligned}
$$

VII. Zwei von einander verschiedene Gruppen $[r],\left[r^{\prime}\right]$, welche in der Beziehung $K_{r, r^{*}} \equiv 0$ stehen, enthalten, für $p>1,4 h_{p-2}$ ungerade und $4 S_{p-2}$ gerade Charakteristiken gemeinsam. Dieselben treten in jeder der beiden Gruppen gepaart auf und je 4 der ungeraden ordnen sich zusammen, ebenso je 4 der geraden. In drei Gruppen $[r],\left[r^{\prime}\right],\left[r r^{\prime}\right]$, für welche $K_{r, r} \equiv 0$, 7ommt jede Charaliteristiti einmal oder dreimal vor, also die zweien gemeinsamen auch in der dritten.

Denn sei $(\alpha)$ irgend eine Charakteristik; so wird die Summe:

$$
\begin{aligned}
& \sum\left(n^{r \alpha} m^{r \alpha}+n^{r^{\alpha}} m^{r^{\alpha}}+n^{r r^{\alpha}} m^{r r^{\prime} \alpha}\right) \equiv \\
\equiv & \sum\left\{n^{r} m^{r}+n^{r^{\prime}} m^{r^{\prime}}+\left(n^{r} m^{r}+n^{r} m^{r^{\prime}}\right)+\left(n^{r} m^{r^{\prime}}+n^{r^{\prime}} m^{r}\right)\right\} \\
+ & \sum\left\{\left(n^{r r^{\prime}} m^{\alpha}+n^{\alpha} m^{r r^{\prime}}\right)+\left(n^{r} m^{\alpha}+n^{\alpha} m^{r}\right)+3 n^{\alpha} m^{\alpha}\right\} \equiv \\
\equiv & \sum n^{\alpha} m^{\alpha} \quad(\bmod .2),
\end{aligned}
$$

d. h. es sind entweder eine oder drei der Charakteristiken $(r \alpha),\left(r^{\prime} \alpha\right)$, $\left(r r^{\prime} \alpha\right)$ mit $(\alpha)$ zugleich gerade, bezüglich ungerade, was der letate Theil von VII. ist. Ferner sind die zweien der Gruppen gemeinsamen Charakteristiken in jeder der Gruppen nur gepaart enthalten, nach Satz IV.; man hat also die folgende Bildungsweise, wenn $(\alpha),(\beta),\left(\alpha^{\prime}\right),\left(\beta^{\prime}\right)$ alle gerade oder alle ungerade sind:

$$
\begin{aligned}
{[r] } & \equiv(\alpha),\left(\beta^{\prime}\right) \equiv\left(\alpha^{\prime}\right),\left(\beta^{\prime}\right) \equiv \cdots, \\
{\left[\gamma^{\prime}\right] } & \equiv(\alpha),\left(\alpha^{\prime}\right) \equiv(\beta),\left(\beta^{\prime}\right) \equiv \cdots, \\
{\left[r r^{\prime}\right] } & \equiv(\beta),\left(\alpha^{\prime}\right) \equiv(\alpha),\left(\beta^{\prime}\right) \equiv \cdots,
\end{aligned}
$$

wo sich denn $(\alpha),(\beta),\left(\alpha^{\prime}\right),\left(\beta^{\prime}\right)$ zusammenordnen.

Sei endlich $\mu$ die Anzahl ungerader, $v$ die Anzahl gerader Charakteristiken, welche engleich in $[r]$ und $\left[r^{\prime}\right]$, also auch in $\left[r r^{\prime}\right]$, enthalten sind, so folgt daraus, dass jede Charakteristik in $[r],\left[r^{\prime}\right],\left[r r^{\prime}\right]$ einmal oder dreimal auftritt: 
woraus

$$
\begin{aligned}
& \mu+6\left(R_{p-1}-\frac{\mu}{2}\right)=R_{p}, \\
& \nu+6\left(S_{p-1}-\frac{\nu}{2}\right)=S_{p}
\end{aligned}
$$

$$
\mu=4 R_{p-2}, \quad \nu=4 S_{p-2},
$$

womit VII. vollständig bewiesen ist.

VIIl. $Z u$ einer gegebenen Gruppe [r] giebt es noch $2^{2 p-1}$ Gruppen $[s]$, für welche $K_{r, s} \equiv 1$, und $2^{2 p-1} \ldots 2$ Gruppen $\left[r^{\prime}\right]$ (wenn man $[r]$ selbst ausschliesst), für welche $K_{r, r^{\circ}} \equiv 0$. Es giebt $\frac{1}{6} K_{2 p}$ Tripel von Gruppen der Art $[r],[s],[r s]$, wo $K_{r, s} \equiv 1$.

Denn sei $(\alpha),(\beta)$ ein Paar in $[r]$ enthaltener gerader Charakteristiken; eine Gruppe $[s]$ enthält entweder $(\alpha)$ oder $(\beta)$, und alle $[s]$ werden gebildet, indem man $(\alpha)$ sowohl als $(\beta)$ mit allen $2^{2 p-2}$ nicht in $[r]$ enthaltenen geraden Charalteristiken, $(\gamma),\left(\gamma^{\prime}\right), \cdots$, zusammensetzt $z u$

$$
[\alpha \gamma],[\beta \gamma],\left[\alpha \gamma^{\prime}\right],\left[\beta \gamma^{\prime}\right], \cdots,
$$

was $2 \cdot 2^{2 p-2}$ Gruppen [s] liefert. Die übrigen Gruppen, ausser $[r]$ selbst, sind Gruppen [r'].

IX. Drei Gruppen $[r],[s],[t]$, für welche

$$
K_{r, s} \equiv 1, \quad K_{r, t} \equiv 1, \quad K_{s, t} \equiv 1,
$$

whe dass $[t]=[r s]$ ist, haben $2^{2 p-1}$ gerade und $2^{2 p-1}$ ungerade Charakteristiken gemein.

Sei

$$
\begin{aligned}
& {[r] \equiv\left(\alpha_{1}\right),\left(\beta_{1}\right) \equiv \cdots \equiv\left(\alpha_{S_{p-1}}\right),\left(\beta_{s_{p-1}}\right),} \\
& {[s] \equiv\left(\alpha_{1}\right),\left(\gamma_{1}\right) \equiv \cdots \equiv\left(\alpha_{S_{p-1}}\right),\left(\gamma_{s_{p-1}}\right),}
\end{aligned}
$$

wo die $(\alpha),(\beta),(\gamma)$ alle gerade sind; so folgt:

$$
[r s] \equiv\left(\beta_{1}\right),\left(\gamma_{1}\right) \equiv \cdots \equiv\left(\beta_{s_{p-1}}\right),\left(\gamma_{s_{p-1}}\right) .
$$

Aber es ist $K_{\ell, r s} \equiv 0$. Also enthält, nach VII., [ $\left.t\right] 2 S_{p-2}$ der Grössen $(\beta)$ und $2 S_{p-2}$ der Grössen $(\gamma)$, welche in $[r s]$ enthalten sind, und zwar in $2 S_{p-2}$ Paaren. Die übrigen $2^{2 p-4}$ Paare müssen aber je eine $\operatorname{der}[r]$ und $[s]$ gemeinsamen Charakteristiken $(\alpha)$ enthalten. Ebenso folgt der Satz für die ungreraden Charakteristiken.

X. $Z u$ zwei Gruppen $[r],\left[r^{\prime}\right]$, für welche $K_{r, r^{\prime}} \equiv 0$, giebt es noch $2^{2 p-2}$ Gruppen $[t]$, für welche

$$
\mathcal{K}_{r, t} \equiv K_{r^{\prime}, t} \equiv 1 \text {. }
$$

Seien nämlich $(\alpha),(\beta)$ zwei gerade Charakteristiken, welche ein Paar der Gruppe $[r]$ bilden und zugleich in $\left[r^{\prime}\right]$ enthalten sind. Eine Gruppe $[t]$ muss dann entweder $(\alpha)$ oder $(\beta)$ enthalten, und alle $[t]$ werden gebildet, wenn man einmal $(\alpha)$, sodann $(\beta)$, mit allen geraden Charakteristiken zusammensetzt, welche weder in $[r]$, noch in $\left[r^{\prime}\right]$ 
enthalten sind. Von diesen letzteren Charakteristiken $(\gamma)$ giebt es, wie aus VII. folgt, $2^{2 p-3}$; also sind die Gruppen

d. h. die $[t]$ an Zahl $2^{3 p-2}$.

$$
[\alpha \gamma],[\beta \gamma]
$$

Diese Sātze lassen sich nach mehreren Seiten hin vervollständigen. Ich verfolge indessen nur eine Richtung weiter, welche zum Hauptsatze meiner Theorie führt.

\section{$\$ 3$.}

Zurückführung der Theorie der $p$-reihigen Charakteristiken auf die der $(p-1)$-reihigen.

XI. Es giebt Substitutionen, welche irgend zwei Gruppen $[r],[s]$, für welche $K_{r, s} \equiv 1$, überfüleren in irgend awei andere Gruppen [r'], [s'], für welche $K_{r^{\prime}, x^{\prime}} \equiv 1$.

Zunächst ist klar, dass durch eine Substitution die Beziehung $K_{r, s}$ zwischen zwei Gruppen [r], [s] nicht verändert wird, nach der in VI. und VII. gegebenen Bedeutung der Zahl $K_{r, s}$ für [r] und [s]. Man mache also zuerst, nach dem am Schlusse von \$1. Entwickelten, eine Substitution, durch welche die Gruppe $\left[s^{\prime}\right]$ in $[s]$ übergeführt wird; führt dieselve Substitution $\left[r^{\prime}\right]$ in $\left[r_{1}\right]$ ïber, so hat man auch $K_{r_{1}, s} \equiv 1$. Es bleibt dann die Aufgabe, die Gruppen

überzuführen in

$$
\left[r_{1}\right],[s], \quad \text { wo } K_{r_{1}, s} \equiv 1 \text {, }
$$

$$
[r],[s], \quad \text { wo } K_{r, s} \equiv 1 \text {. }
$$

Entweder ist nun $K_{r, r_{s}} \equiv$ 1. Daun wird die Ueberfübrung unmittelbar durch die Substitution $\left\{r r_{1}\right\}$ geleistet; denu wegen $K_{r} r_{1, s}=0$ bleibt dabei $[s]$. unverändert.

Oder es ist $K_{r, r_{s}} \equiv 0$. Dann bestimme man zunächst eiven Zahlencomplex $[\emptyset]$, für welchen

$$
K_{r, t} \equiv K_{r_{1}, t} \equiv K_{s, t} \equiv 1,
$$

was nach dem folgenden Satze inmer geschehen kann. Macht man aber die Substitutiouen $\left\{r_{1} t\right\},\{r t\}$ hintereinander, so erhält man aus $\left[r_{1}\right]$ zuerst $[t]$, sodann $[r]$, während $[s]$ beide Male unverändert bleibt.

- Der hierbei benutzte Satz lautet so:

\section{Zu gegebenen Gruppen}

für welche:

$$
[r],\left[r_{1}\right],[s],
$$

$$
K_{r, r_{1}} \equiv 0, \quad K_{r, s} \equiv K_{r_{2}, s} \equiv 1 \text {, }
$$

gielt es $2^{2 p-3}$ Gruppen [t], für welche:

$$
K_{r, t} \equiv K_{r_{1}, t} \equiv K_{d, t} \equiv 1 \text {. }
$$


Denn seien, genau wie bei X., $(\alpha),(\beta)$ zwei gerade Charakteristiken, welche ein Paar der Gruppe $[r]$ bilden und zugleich in $\left[r_{1}\right]$ enthalten sind. Die Gruppe $[s]$ wird daun etwa von der Form

$$
[s]=\left[\alpha \gamma_{\mathrm{t}}\right]
$$

sein, wo $\left(\gamma_{1}\right)$ eine derjenigen geraden Charakteristiken $(\gamma)$ ist, welche weder in $[r]$, noch in $\left[r_{1}\right]$ enthalten sind. Die Gruppen $[t]$ sind danu, nach X. und VI., in den Formen euthalten:

$$
[\beta \delta],\left[\alpha \delta^{\prime}\right] \text {, }
$$

wo $(d)$ irgend eine solche der oben mit $(\gamma)$ bezeichneten Charakteristiken ist, die zugleich in [s] enthalten ist, $\left(\delta^{\prime}\right)$ irgend eine solche der Charakteristiken $(\gamma)$, die nicht in $[s]$ enthalten jst. Nun giebt es $2^{2, p-3}$ Charakteristiken $(\gamma)$, d. h. ebensoviele $(\delta)$ und $\left(\delta^{\prime}\right)$ zusammengenommen; also ebenso viele Gruppen $[\beta \delta],\left[\alpha \delta^{\prime}\right]$ oder $[t]$, was der Sitz XII. ist. - Man kann noch bemerken, dass es $2 S_{p-2}$ Charakteristiken $(\delta)$ giebt. -

Der Satz XI. lässt eine sehr wichtige Anwendung zu. Sei

$$
\left[r^{\prime}\right]=\left[\begin{array}{ccccc}
0 & 0 & \cdots & 0 & 1 \\
0 & 0 & \cdots & 0 & 0
\end{array}\right], \quad\left[s^{\prime}\right]=\left[\begin{array}{ccccc}
0 & 0 & \cdots & 0 & 0 \\
0 & 0 & \cdots & 0 & 1
\end{array}\right],
$$

so sind alle Charakteristiken von der Form:

$$
\left(\begin{array}{c}
n_{1}^{\alpha} n_{2}^{\alpha} \cdots n_{p-1}^{\alpha} 0 \\
m_{1}^{\alpha} m_{2}^{\alpha} \cdots m_{p-1}^{\alpha} 0
\end{array}\right)
$$

sowohl in [ $\left.r^{\prime}\right]$ als in $\left[s^{\circ}\right]$ entbalten und stellen überhaupt alle $R_{p-1}$ ungeraden und $S_{y-1}$ geraden Charakteristiken vor, welche $\left[r^{\prime}\right]$ und $\left[s^{\prime}\right]$, für die $K_{r^{\prime}, r^{\prime}} \equiv 1$, gemeinsam sind. Die Relationen, welche diese Charakteristiken untereinander eingehen, sind genau dieselben, wie diejenigen, welche zwischen den $R_{p-1}$ ungeraden und $S_{p-1}$ geraden $(p-1)$-reihigeu Charakteristiken

$$
\left(\begin{array}{c}
n_{1}^{c} \cdot n_{2}^{\alpha} \cdots n_{p-1}^{\alpha} \\
m_{1}^{\alpha} m_{2}^{\alpha} \cdots m_{p-1}^{\alpha}
\end{array}\right)
$$

überhaupt existiren, da bei jeder beliebigen Summirung jener Charakteristiken inmer wieder eine derselben Art erhalten wird. Aber unsere Substitutionen lassen diese Relationen bestehen, und erlauben nach XI. die Gruppen $\left[r^{\prime}\right],\left[s^{\prime}\right]$ in irgend zwei Gruppen $[r]$, [s] überzuführen, für welche $K_{r, s} \equiv 1$. Dieses liefert sogleich den Hauptsatz unserer Theorie:

XIII. Die $R_{p-1}$ ungeraden und $S_{p-1}$ geraden p-reitigen Charalteristiken, welche in irgend awei Gruppen $[r],[s]$, für die $K_{r, s} \equiv 1, g t-$ meinsam enthalten sind, stehen unter einander genau in densclben Be- 
ziehungen, wie die ülerhaupt existirenden $R_{\mu-1}$ ungeraden und $S_{p-1}$ geraden $(p-1)$-reihigen Charaliteristiken.

Dieser Satz kann nun zu einer Eintheilung aller Gruppen benutat werden, nit Hülfe der in dem folgenden Satze ausgesprochenen Erläuterung:

XIV. Bei dem in XIII. gegebenen Entsprechen treten an Stelle der bei den $(p-1)$-reihigen Charakteristiken vorhandenen $2^{2(p-1)}-1$ Gruppen $\left[r_{1}^{\prime}\right]$ diejenigen $2^{2(p-1)}-1$ Gruppen $\left[r_{1}\right]$, für welche

$$
K_{r, r_{2}} \equiv K_{s_{2} \tau_{s}} \equiv 0 \text {. }
$$

Ebenso wie der Charaliter tes Geraden oder Ungeraden der eigentlichent Charakteristitien geht dabei auch die Beziehung $K_{r_{i}, s_{i}^{\prime}}$ zuoischen zwei Gruppen $\left[r_{1}{ }^{\prime}\right],\left[s_{1}{ }^{\prime}\right]$ aus den $(p-1)$-reihigen Charalteristiken in dieselbe Beziehung $K_{r_{1}, s_{1}}$ zwischen den unter den $p$-reihigen Charakteristiten entsprechenden Gruppen $\left[r_{1}\right],\left[s_{1}\right]$ über.

Denn seien die Zerlegungen von $[r]$ und $[s]$ in Paare gerader Charakteristiken:

$$
\begin{aligned}
& {[r] \equiv\left(\alpha_{1}\right),\left(\beta_{1}\right) \equiv \cdots \equiv\left(\alpha_{S_{p-1}}\right),\left(\beta_{s_{p-1}}\right),} \\
& {[s] \equiv\left(\alpha_{1}\right),\left(\gamma_{1}\right) \equiv \cdots \equiv\left(\alpha_{S_{p-1}}\right),\left(\gamma_{s_{p-1}}\right) .}
\end{aligned}
$$

Eine Gruppe $\left[r_{1}\right]$ nun, für welche

$$
K_{r_{i} r_{s}} \equiv K_{s_{1} r_{\mathrm{s}}} \equiv 0 \text {, }
$$

enthält, nach VII., $2 S_{p-2}$ der Grössen $(\alpha)$, und die $(\beta)$ und $(\gamma)$ mit gleichem Index. Aber dabei muss nothwendig in $\left[r_{1}\right]$ ein $\left(\alpha_{\rho}\right)$ immer mit einem $\left(\alpha_{\sigma}\right)$ gepaart sein; denn andernialls wäre $\left[r_{1}\right] \equiv\left[\alpha_{\rho} \beta\right]$ und $\left(\beta_{\sigma}\right)$ müsste dann, wegen $K_{s, r}, \equiv 0$, auch in $[s]$ enthalten sein; was nicht stattfindet. Man erhält daher alle Gruppen $\left[r_{1}\right]$, indem man irgend zwei der $S_{p-1}$ geraden Charaliteristiken $\left(\alpha_{Q}\right)$, welche $[r]$ und $[s]$ gemeinsam sind, mit einander combinirt. Dies giebt, indem man nach Satz XIII. den für die $(p-1)$-reihigen Charakteristiken auszudrückenden Satz II. anwendet, im Ganzen $2^{2(p-1)}-1$ Gruppen $\left[r_{1}\right]$. In der That liefern je $S_{p-2}$ Combinationen der $S_{p-1}$ Grössen $(\alpha)$ dieselbe Gruppe $\left[r_{1}\right]$, nach VII., und man hat also

$$
\frac{s_{p-1}\left(S_{p-1}-1\right)}{2 \cdot S_{p-2}}=2^{2(p-1)}-1
$$

Gruppen $\left[r_{1}\right]$. Der letzte Theil des Satzes XIV. ist eine unmittelbare Folge der Bedeutung der Zahl $K_{r_{1}, s_{1}}$ für zwei Gruppen $\left[r_{1}\right],\left[s_{1}\right]$, wie sie in VI. und VII. angeführt ist, nānlich, dass ron irgend einem Paar $\left(\alpha_{1}\right),\left(\alpha_{2}\right)$ von $\left[r_{1}\right]$ für $K_{r_{1}, s_{4}} \equiv 1$ nur eine Charakteristik in $\left[s_{1}\right]$ enthalten ist, für $K_{r_{1}, s_{1}} \equiv 0$ aber beide oder keine.

Man kann hiernach alle Sätze des $\$ 2$. unmittelbar so verallgemeinern: 
XV. Die $S_{p-1}$ aweien Gruppen $[r],[s]$, für die $K_{r, s}=1$, gemeinsumen geraden Charakteristiken seien hier mit $(a)$, die $R_{p-1}$ gemeinsamen ungeraden mit $(b)$ bezeichnet. Jede der $2^{2(p-1)}-1$ Gruppen $\left[r_{1}\right]$, für die

$$
K_{r r_{1}} \equiv K_{s_{1} r_{2}} \equiv 0 \text {, }
$$

enthält dann $S_{p-2}$ Paare der (a) und $R_{p-2}$ Paare der $(b)$.

Zwei Gruppen $\left[r_{1}\right],\left[s_{1}\right]$, für welche

$$
K_{r, r_{1}} \equiv K_{s, r_{1}} \equiv 0, \quad K_{r, s_{1}} \equiv K_{s, s_{1}} \equiv 0,
$$

und zugleich

$$
K_{r_{1}, s_{1}} \equiv 1 \text {, }
$$

enthalten $S_{p-2}$ der (a) und $R_{p-2}$ der (b) gemeinsam, und zwar ungepaart in $\left[r_{1}\right]$ und in $\left[s_{1}\right]$. Drei solche Gruppen $\left[r_{1}\right],\left[s_{1}\right],\left[r_{1} s_{1}\right]$ enthalten lieine Charahteristik (a) oder (b) gemeinsam.

Zwei Gruppen $\left[r_{1}\right],\left[r_{1}^{\prime}\right]$, für welche

$$
\begin{gathered}
K_{r, r_{1}} \equiv K_{s, r_{1}} \equiv \dot{0}, \quad K_{r, r_{i}} \stackrel{\cdots}{=} K_{s, r_{i}^{\prime}} \equiv 0, \\
K_{r_{s}, r_{1}^{\prime}} \equiv 0,
\end{gathered}
$$

enthalten, für $p>2,4 S_{p-3}$ der (a) und $4 R_{p-3}$ der (b) gemeinsam, die in jeder der beiden Gruppen gepaart auftreten.

$Z u$ einer gegebenen Gruppe $\left[r_{1}\right]$ giebt es noch $2^{2(p-1)-1}$ Gruppen der obigen Art $\left[s_{1}\right]$ und $2^{2(p-1)-1}-2$ Gruppen der Art $\left[r_{1}^{\prime}\right]$. Etc. etc.

\section{$\S 4$.}

Zurückführung anf $(p-\mu)$-roihige Charakteristiken.

Wendet man die Schlüsse des $\S 3$. auf die Charakteristiken von weniger als $p$-Reihen an, so ergiebt XIII. und XIV. unmittelbar die Zurückführung der Theorie der $p$-reihigen Clarakteristiken auf die der $(p-\mu)$-reihigen durch folgenden Satz:

XVI. $2 \mu$ Gruppen

für welche

$$
\begin{array}{cc}
{\left[r_{1}\right],} & {\left[s_{1}\right] ;} \\
{\left[r_{2}\right],} & {\left[s_{2}\right] ;} \\
\vdots & \vdots \\
\left\lfloor r_{\mu}\right], & {\left[s_{\mu}\right],}
\end{array}
$$

dagegen

$$
K_{r_{i}, s_{i}} \equiv 1 \text {, }
$$

$$
K_{r_{i}, r_{k}} \equiv K_{s_{i}, s_{k}} \equiv K_{r_{i}, s_{k}} \equiv 0, \quad\left(k_{i} i_{i}\right),
$$

enthalten $S_{p-\mu}$ gerade und $R_{p-\mu}$ ungerude p-reihige Charakteristiken gemeinsam, welche in keiner der Gruppen gepaart auftreten. Die ersteren seien in diesem Paragraphen als $(a)$, die letzteren als $(b)$ bezeichnet. Für diese gemeinsamen Charakteristiken existiren genau diesellen Re- 
lationen, wie für die überhaupt vorhandenen $S_{p-\mu}$ geraden und $R_{p-\mu}$ ungeraden $(p-\mu)$-reihigen Charahteristitien. Insbesondere treten an Stelle der bei diesen existirenden $2^{2(p-\mu)}-1$ Gruppen die durch $Z u$ sammensetzung je zweier der (a) oder je zweier der (b) gebildeten $2^{2(p-\mu)}-1$ Gruppen, d. h. alle Gruppen $\left[r_{\mu+1}\right]$, für welche

$$
K_{r_{i}, r_{\mu+1}} \equiv K_{s_{i}, r_{\mu+1}} \equiv 0 \text {, }
$$

wo $i$ alle Werthe von 1 bis $\mu$ anzunehmen hat.

Man hat hiernach aus der Theorie der $(p-\mu)$-reihigen Charakteristiken sogleich die weiteren Sätze:

XVII. Jede der in XVI. definirten Gruppen $\left[r_{u+1}\right]$ enthält $S_{p-\mu-1}$ Paare aus den (a) und $R_{p-u-1}$ Paare aus den (b). - Zwei solche Gruppen, $\left[r_{\mu+1}\right]$ und $\left[s_{\mu+1}\right]$, für die zugleich $K_{r_{\mu+1}, s_{\mu+1}} \equiv 1$, enthalten $S_{p-\mu-1} \operatorname{der}(a)$ und $R_{p-\mu-1}$ der (b) gemeinsam, ungepaart in $\left[r_{\mu+1}\right]$ und in $\left[s_{\mu+1}\right]$; und zu einer gegebenen Gruppe $\left[r_{\mu+1}\right]$ giebt es noch $2^{2(p-\mu)-1}$ solcher Gruppen $\left[s_{\mu+1}\right]$. - Zwei solche Gruppen, $\left[r_{\mu+1}\right]$ und $\left[r_{\mu+1}^{\prime}\right]$, für die $K_{r_{\mu+1}, r_{\mu+1}^{\prime}} \equiv 0$, enthalten, für $p>\mu+1,4 S_{p-\mu-2}$ der (a) und $4 R_{p-\mu-2}$ der $(b)$ gemeinsam, die in jeder der beiden Gruppen gepaart auftreten; und jede der $(a),(b)$ tritt entweder in einer der drei Gruppen $\left[r_{\mu+1}\right],\left[r_{\mu+1}^{\prime}\right],\left[r_{u+1} r_{\mu+1}^{\prime}\right]$ oder in allen dreien zugleich auf.

XVIII. Setat man die in XVI. definirten Charakteristiken (a) und (b) in irgend einer Weise zu Gruppen zusammen:

$$
\left[a_{\rho} a_{\sigma}\right],\left[b_{\varphi} b_{\sigma}\right],\left[a_{\ell} b_{e}\right],\left[a_{\ell} b_{\sigma}\right],\left[a_{Q} a_{\sigma} b_{\tau}\right], \cdots,
$$

so erhält man nur solche Gruppen, welche $z u$ den in XVI. definirten $\left[r_{\mu+1}\right]$ gehören. Ond setzt man die $(a)$ und $(b)$ in ungeraller Anzahl $z u$ neuten Charaliteristiken zusammen, so erhält man immer nur wieder solche aus der Reihe der $(a),(b)$. Jede dieser Charalteristiken $\left(a_{0}\right)$ lässt sich auf melorfache Art in die Form $\left(b_{o} b_{\tau} b_{b}\right)$ setzen; und umgekehrt jede $\left(b_{\varphi}\right)$ auf mehrfache Art in die Form $\left(a_{\sigma} a_{\tau} a_{\zeta}\right)$. Für $\mu=p-2$ ist $\left(b_{\sigma} b_{z} b_{\xi}\right)$, wenn $\sigma, \tau, \xi$ von einander verschieden sind, immer gerade. Die Summe der Charakteristiken (a) ist 0 , ebenso die der (b), ausgenommen für $\mu=p-1$.

Der letzte Theil des Satzes beweist sich so: Seien $\left[r_{\mu+1}\right],\left[r^{\prime}{ }_{\mu+1}\right]$, wo $K_{r_{\mu+1}, r_{\mu+1}^{\prime}} \equiv 0$, zwei der Gruppen $\left[r_{\mu+1}\right]$ des Satzes XVI.; da nach XVII. jede der Grössen $(a)$ in $\left[r_{\mu+1}\right],\left[r_{\mu+1}^{\prime}\right],\left[r_{\mu+1} r_{\mu+1}^{\prime}\right]$ einmal oder dreimal auftritt, so folgt durch Summirung der $S_{p-\mu-1}$ Paare von $\left[r_{\mu+1}\right]$, welche die Grōssen $(a)$ enthalten, zu den analogen Paaren von $\left[r_{\mu+1}^{\prime}\right]$ und $\left[r_{\mu+1} r_{\mu+1}^{\prime}\right]$ :

$$
\Sigma(a)=\left(r_{\mu+1}\right)+\left(r_{\mu+1}^{\prime}\right)+\left(r_{\mu+1} r_{\mu+1}^{\prime}\right)=0 ;
$$

und ebenso für die Summe aller $(b)$. Nur fïr $\mu=p-1$ lassen sich aus den $S_{1}=3$ geraden und $R_{1}=1$ ungeraden Charakteristik nicht 
zwei Gruppen $\left[r_{p}\right],\left[r_{p}^{\prime}\right]$ bilden, für welehe $K_{r p}, r_{p}^{\prime} \equiv 0$; und alsdanı wird $\Sigma(a)+(b)=0$.

Für $\mu=p-2$ hat man $S_{2}=10$ gerade und $R_{2}=6$ ungerade Charakteristiken, $(a)$ bez. $(b)$, wo $\Sigma(a)=0, \Sigma(b)=0$. Sei nun $\left(a_{0}\right)$ irgend ein $(a),\left(b_{a}\right)$ irgend ein $(b)$. Die Gruppe $\left[a_{o} b_{\sigma}\right]$ enthält danu nach XVII. ein Paar ungerader Charakteristiken $(b):\left(b_{\tau}\right)+\left(b_{5}\right)$, so dass für jede der 10 Charakteristiken $\left(a_{e}\right)$ :

wenn

$$
\left(a_{e}\right)=\left(b_{\sigma} b_{\tau} b_{\zeta}\right)=\left(b_{\sigma^{\prime}} \cdot b_{\boldsymbol{x}^{\prime}} \cdot b_{\xi^{\prime}}\right),
$$

$$
\left(b_{\sigma}\right)+\left(b_{z}\right)+\left(b_{\zeta}\right)+\left(b_{\sigma}\right)+\left(b_{x^{*}}\right)+\left(b_{\zeta}\right)=0 .
$$

Da umgekehrt aus den sechs Grössen $(b)$ nur 10 ron einander verschiedene Combinationen zu 3 möglich sind, so folgt, dass jede solche Combination ein (a) liefert.

Neben diese Sätze über die Charakteristiken $(a),(b)$, welche den $2 \mu$ Gruppen von XVI. gemeinsam sind, stellt sich nun eine andere Reihe von Sützen über diejenigen Gruppen, welche diese $(a)$ und $(b)$ ebenfalls sämntlich enthalten oder welche keine von ihnen entbalten. Um diese zu bilden, setzen wir die $2 \mu$ Gruppen von XVI. in allen möglichen Combinationen durch Addition zu neuen Gruppen zusammen. Wir unterscheiden dann, ob in dieser Zusammensetzung einer solchen neuen Gruppe die beiden zusammengehörigen Gruppen $\left[r_{t}\right],\left[s_{i}\right] \mathrm{zu}-$ gleich vorkommen, oder nur eine derselben. Jedes Paar $r_{i} s_{i}$, das in der Zusammensetzung auftritt, sei ersetzt durch

$$
r_{i} s_{i}=t_{i}, \quad(i=1,2, \cdots \mu) .
$$

Wir haben dann den Satz:

XIX. Diejenigen aus den $2 \mu$ Gruppen von XVI. zusammengesetzten Gruppen, in deren Ausdruck eine gerade Anzahl der $r_{i} s_{i}=t_{i}$ vorkommt, seien mit $[u]$, die übrigen, bei welchen diese Anzahl ungerade ist, mit $[v]$ bezeichnet. Die Charaliteristiken $(a)$ und $(b)$ des Satzes XVI. sind dann zugleich alle in sämmtlichen Gruppen [u], und zwar ungepaart, enthalten, und in keiner anderen Gruppe. Die Gruppen $[v]$, und nur diese, enthalten keine der Charaliteristiken (a) (b). Von den Gruppen [u] giebt es $S_{\mu}-1$, von den Gruppen [v] giebt es $R_{\mu}$.

Denn die Charakteristiken (a) (b) kommen, da sie in $\left[r_{i}\right]$ und $\left[r_{k}\right]$ enthalten sind, wegen $K_{r_{t}, r_{k}} \equiv 0$ nach VII. auch in $\left[r_{i} r_{k}\right]$ vor; ebenso, da sie in $\left[r_{i} r_{k}\right]$ und $\left[r_{l}\right]$ enthalten sind, auch in $\left[r_{i} r_{k} r_{l}\right]$, etc.; allgemein in allen Gruppen

$$
\left[r_{s} r_{k} r_{l} r_{m} \cdots\right] \text { und }\left[s_{i} s_{k} s_{l} s_{m} \cdots\right] \text {. }
$$

Eine Gruppe $[u]$ oder $[v]$ ist nun von der Form:

$$
\left[t_{i} t_{k} \cdots r_{z}, r_{k^{\prime}} \cdots s_{i} s_{k^{\prime \prime}} \cdots\right] \text {, }
$$

entsteht also durch Summirung der beiden Gruppen 


$$
\left[r^{\prime}\right]=\left[r_{i} r_{k} \cdots r_{i} \cdot r_{k^{\prime}} \cdots\right], \quad\left[s^{\prime}\right]=\left[s_{i} s_{k} \cdots s_{i} s_{k^{\prime \prime}} \cdots\right],
$$

wo die $i, k, \cdots, i^{\prime}, k^{\prime}, \cdots, i^{\prime \prime}, k^{\prime \prime}, \ldots$ alle von einander verschieden sind. Für eine Gruppe [u] stehen diese beiden Gruppen $[r],\left[s^{\prime}\right]$ in der Beziehung

$$
K_{r^{\prime}, s^{\prime}} \equiv K_{r_{i}, s_{t}}+K_{r_{k}, s_{k}}+\cdots \equiv 0
$$

daher enthäIt $[u]$ alle $\left[r^{\prime}\right]$ und $\left[s^{\prime}\right]$ gemeinsamen Charakteristiken, also auch alle $(a),(b)$. Für eine Gruppe $[v]$ stehen aber $\left[r^{\prime}\right],\left[s^{\prime}\right]$ in der Beziebung

$$
K_{r_{i} v^{r}} \equiv K_{r_{i}, s_{i}}+K_{r_{k}, s_{k}}+\cdots \equiv 1
$$

und nach VI. kann keine der in $\left[r^{\prime}\right],\left[s^{\prime}\right]$ zugleich enthaltenen Charakteristiken, also keine der $(a),(b)$, in $[v]$ enthalten sein. In den Gruppen $[u]$ sind die Grössen $(a),(b)$ nur ungepaart enthalten, da Verbindungen dieser Grössen nach XVIII. nur zu Gruppen $\left[r_{k+1}\right]$ führen.

Die Gruppen $[u]$ sind alle von einander verschieden, ebenso die $[v]$. Denn wären zwei solche Gruppen einander gleich, so wäre der Ausdruck, der durch deren Summirung entsteht, $=0$ :

$$
\left[t_{t} t_{k} \cdots r_{i^{\prime}} r_{k^{\prime}} \cdots s_{i}{ }^{\prime \prime} s_{k^{\prime \prime}} \cdots\right]=0 \text {. }
$$

Aber dieser Ausdruck kann nicht 0 sein, da er immer in die Summe zweier solcher Ausdrücke $\left[r^{\prime}\right],\left[s^{\prime}\right]$ zerlegt werden kann, für die $K_{r^{\prime}} s^{\prime} \equiv \mathbf{1}$; etwa in

$$
\left[r_{i}\right]+\left[s_{i} t_{k} \cdots r_{i}, r_{k} \cdots s_{i} s_{k^{\prime \prime}} \cdots\right] .
$$

Um alle Gruppen $[u]$ and $[v]$ zu erhalten, bilde man zunächst diejenigen derselben, $\left[u^{\prime}\right],\left[v^{\prime}\right]$, welche durch Zusammensetzung der Gruppenpaare

$$
\left[r_{1}\right],\left[s_{1}\right] ;\left[r_{2}\right],\left[s_{2}\right], \cdots ;\left[r_{\mu-1}\right],\left[s_{\mu-1}\right]
$$

entstehen. Angenommen, dieselben seien an Anzahl $S_{\mu-1}-1$, be\%. $R_{\mu-1}$, was für $\mu=2$ jedenfalls richtig ist. Man erhält dann alle $[u]$ in den Formen:

$$
\left[u^{\prime}\right],\left[u^{\prime} r_{\mu}\right],\left[u^{\prime} s_{\mu}\right],\left[r_{\mu}\right],\left[s_{\mu}\right],\left[v^{\prime} r_{u} s_{\mu}\right],
$$

also im Ganzen

$$
3\left(S_{\mu-1}-1\right)+2+R_{\mu-1}=S_{\mu}-1
$$

Gruppen $[u]$; und alle Gruppen [v] in den formen:

das sind

$$
\left[v^{\prime}\right],\left[v^{\prime} r_{\mu}\right],\left[v^{\prime} s_{\mu}\right],\left[r_{\mu} s_{\mu}\right],\left[u^{\prime} r_{\mu} s_{\mu}\right] \text {, }
$$

Gruppen $[v]$.

$$
3 R_{\mu-1}+1+\left(S_{\mu-1}-1\right)=R_{\mu}
$$

Dass nur die Gruppen $[u]$ und $[v]$ die Eigenschaft halen, alle oder keine der $(a)$ und $(b)$ zu enthalten, wird aus dem Satze XX. gefolgert werden:

XX. Die Charahteristiken $(a),(b)$ und Gruppen $\left[r_{\mu+1}\right]$ seien wieder 
durch XVI., die Gruppen [u], [v] durch XIX. definirt. Dann sind die Charalteristiken

$$
(a),(u a),(v b)
$$

alle von einander verschicden und stellen alle $S_{p}$ geraden Charakteristiken vor; ebenso sind die

$$
(b),(u b),(v a)
$$

alle von einander verschieden und stellen alle $R_{p}$ ungeraden Charak. teristiken vor. Die Gruppen

$$
[u],[v],\left[r_{\mu+1}\right],\left[u r_{\mu+1}\right],\left[v r_{\mu+1}\right]
$$

sind ebenfalls alle von einunder verschieden und stellen alle $2^{2 p}-1$ Gruppen vor.

Zunächst nämlich sind die in XX. angegebenen Charakteristiken von einander verschieden; denn wäre etwa

so folgte:

$$
\left(u a_{\rho}\right)=\left(v b_{o}\right)
$$

$$
[u v]=\left[a_{Q} b_{\sigma}\right]=\left[r_{\mu+1}\right]
$$

nach XVIIl. Aber diese Gleichheit ist unmöglich, weil $[u v]$ zu den $[u]$ oder $[v]$ gehört, $\left[r_{\mu+1}\right]$ aber nach XVII. nicht. Ebenso folgt die Verschiedenheit der Gruppen; denn etwa aus

$$
\left[u r_{\mu+1}^{\prime}\right],=\left[v r_{\mu+1}^{\prime \prime}\right]
$$

ergäbe sich

$$
[u v]=\left[r_{\mu+1}^{\prime} r_{\mu+1}^{\prime \prime}\right]=\left[r_{\mu+1}\right] \text {, }
$$

was uicht der Fall ist. - Dass man in den in XX. hingeschriebenen formen sodann alle existirenden Charakteristiken ind Gruppen vor sich hat, ergiebt eine einfache Abzählung, mit Benutzung der Relationen:

$$
\begin{aligned}
& S_{x-\mu} S_{\mu}+R_{p-\mu} R_{\mu}=S_{p}, \\
& R_{p-\mu} S_{\mu}+S_{p-\mu} R_{\mu}=R_{p} .
\end{aligned}
$$

Dass, wie XIX. verlangt, die sämmtlichen Gruppen, ausser den $[u]$ und $[v]$, immer einen Theil der $(a)$ und $(b)$ enthalten, folgt so: Von den Gruppen $\left[r_{\mu+1}\right]$ sagt dies Satz XVII. aus; eine Gruppe [ur $\left.r_{\mu+1}\right]$ aber kann nicht alle $(a)$ und $(b)$ enthalten, da dasselbe dann auch, nach VII., wegeu $K_{u, r_{\mu+1}} \equiv 0$, wie in $[u]$, auch in $\left[r_{\mu+1}\right]$ stattfinden müsste. Ebenso enthält eine Gruppe $\left[v r_{\mu+1}\right]$ denjenigen Theil der $(a)$ und $(b)$, welchen $\left[r_{\mu+1}\right]$ enthält, $[v]$ aber nicht enthält, wegen $K_{v, r_{\mu+1}} \equiv 0$ nach VII. ebenfalls nicht. Andere Gruppen, ausser dell $[u]$ und $[v]$ selbst, giebt es aber nach XX. nicht. 


\section{$\S \check{5}$.}

Beispiel. Anzahl der Gruppensysteme. Substitutionsgruppen.

1. Sei zur Abkürzung*) in diesem $\S$ der Ausdruck

$$
\left(\begin{array}{ccc}
n_{1}^{\alpha} n_{2}^{\alpha} \cdots & n_{p}^{\alpha} \\
m_{1}^{\alpha} m_{2}^{\alpha} & \cdots & m_{p}^{\alpha}
\end{array}\right)
$$

wenn darin alle Elemente congruent 0 (mod. 2) sind, mit Ausnahme von $n_{i}^{\alpha}$, mit $\left(\varrho_{i}\right)$, wenn aber alle Elemente mit Ausnahme von $m_{i}^{\alpha}$ congruent 0 sind, mit $\left(\sigma_{i}\right)$ bezeichnet. Die Summe melırerer solcher Charakteristiken $\left(\rho_{i}\right),\left(\rho_{k}\right) \cdots\left(\sigma_{i}\right) \cdots$ wird wie früher mit $\left(\rho_{i} \varrho_{k} \sigma_{i} \cdots\right)$ bezeichnet; und insbesondere soll hierbei überall, wo ein Paar $\left(\rho_{i} \sigma_{i}\right)$ vereinigt auftritt, dasselbe ersetzt werden durch

$$
\rho_{i} \sigma_{i}=\tau_{i}
$$

Wir nehmen dann, wie beim Beweise des Satzes XIII., § 3., an:

$$
\left[r_{1}\right]=\left[\varrho_{p}\right], \quad\left[s_{1}\right]=\left[\sigma_{p}\right]
$$

und fügen für die übrigen Gruppen des Satzes XVI. folgende hinzu:

$$
\begin{array}{cc}
{\left[r_{2}\right]=\left[\varrho_{p-1}\right],} & {\left[s_{2}\right]=\left[\sigma_{p-1}\right] ;} \\
{\left[r_{3}\right]=\left[\varrho_{p-2}\right],} & {\left[s_{3}\right]=\left[\sigma_{p-2}\right] ;} \\
: & \vdots \\
{\left[r_{\mu}\right]=\left[\varrho_{p-\mu+1}\right],} & {\left[s_{\mu}\right]=\left[\sigma_{p-\mu+1}\right] .}
\end{array}
$$

Die in diesen $2 \mu$ Gruppen enthaltenen $S_{p-\mu}$ geraden und $R_{p-\mu}$ ungeraden Charakteristiken $(a)$, bez. $(b)$, sind diejenigen, welche sich aus

$$
\begin{array}{ll}
\left(\varrho_{1}\right), & \left(\varrho_{2}\right), \cdots,\left(\varrho_{p-\mu}\right), \\
\left(\sigma_{1}\right), & \left(\sigma_{2}\right), \cdots,\left(\sigma_{p-\mu}\right)
\end{array}
$$

durch Addition zusammensetzen lassen, wenn man noch $(0)$ binzunimmt. Gerade, $(a)$, sind dabei diejenigen Charakteristiken, in deren Ausdruck eine gerade Zahl der $\varrho_{i} \sigma_{i}=\tau_{i}$ vorkommt, ungerade, $(b)$, die übrigen. Die Gruppen $[u]$ und $[v]$, welche alle $(a)$ und $(b)$ orler keine derselben enthalten, setzen sich aus den

$$
\begin{aligned}
& {\left[\varrho_{p}\right], \quad\left[\varrho_{p-1}\right], \cdots,\left[\varrho_{p-\mu+1}\right],} \\
& {\left[\sigma_{p}\right],\left[\sigma_{p-1}\right], \cdots,\left[\sigma_{p-\mu+1}\right]}
\end{aligned}
$$

durch Addition zusammen, wobei die $[u]$ diejenigen $S_{\mu}-1$ sind, in deren Ausdruek eine gerade Anzahl der $\tau$ enthalten ist, die [v] die $R_{\mu}$ übrigen Gruppen.

Insbesondere bestehen, für $\mu \sim p$, die in den $2 p$ Gruppen enthaltenen Charakteristiken $(a),(b)$ aus der einzigen geraden Charak-

- Vgl. Sehottky in der oben citirten Arbeit, pag. 13.

Mathematische Anzalen. XVI. 
teristik (0); und die sämmtlichen Gruppen zerfallen in zwei Classen $[u]$ und [v] von $S_{p}-1$ und $R_{p}$ Gruppen. -

2. Wir leiten jetzt aus den Sätzen des $\$ 4$. einige Abzählungen her in Bezug auf die Anzahlen solcher Systeme, wie sie in $\S 4$. betrachtet wurden, und in Bezug auf die zugehörigen Substitutionsgruppen.

XXI. Von Systemen von $\mu$ Gruppenpaaren der Art XVI., § 4., gillt es

$$
M_{\mu}=\frac{R_{2 p} \cdot R_{2(p-1)} \cdots R_{2\langle p-\mu+1)}}{2^{\mu} \cdot 1 \cdot 2 \cdot 3 \cdots \mu} .
$$

Die Anzahl derjenigen Systeme dieser Art, welche zugleich alle dieselben $S_{p-, \mu}$ Charahteristiken (a) und $R_{p-\mu}$ Charalteristiken (b) enthalten, beträgt:

$$
\begin{aligned}
N_{\mu} & =\frac{\left(S_{\mu}-1\right) \cdot\left(S_{\mu-1}-1\right) \cdots\left(S_{1}-1\right) \cdot 2^{\mu(\mu-2)}}{1 \cdot 2 \cdot 3 \cdots \mu} \\
& =\frac{R_{\mu} R_{2(\mu-1)} \cdot R_{2(\mu-2)} \cdots R_{4} \cdot R_{2}}{2^{\mu-1} \cdot 1 \cdot 2 \cdot 3 \cdots \mu} .
\end{aligned}
$$

Die Anzahl solcher Complexe von $S_{p-\mu}$ geraden und $R_{p-\mu}$ ungeraden Charaliteristiken $(a),(b)$, welche überhaupt in irgend einem der Systeme der Art XVI., \$ 4. enthalten sind, beträgt:

$$
\frac{M_{\mu}}{N_{\mu}}=\frac{R_{2 p} \cdot R_{2(p-1)} \cdots R_{2(p-\mu+1)}}{2 R_{\mu} \cdot R_{2(\mu-1)} \cdot R_{2(\mu-2)} \cdots R_{4} \cdot R_{2}^{-}}
$$

(und 1 für $\mu=0$ ).

In der That kann man, um ein System der Art XVI., § 4., zu bilden, $\left[r_{1}\right]$ zunächst ganz willkürlich, also auf $2^{2 p}-1$ verschiedene Weisen, $\left[s_{1}\right]$ sodanu [s. VIIl.] auf $2^{2 p-1}$ Weisen wählen; das Paar $\left[r_{1}\right],\left[s_{1}\right]$ also, da man noch $\left[r_{1}\right]$ mit $\left[s_{1}\right]$ vertauschen kann, auf

$$
\frac{\left(2^{2 p}-1\right) \underline{9}^{2 p-1}}{2}=\frac{1}{2} R_{2 p}
$$

Weisen. Ist $\left[r_{1}\right],\left[s_{1}\right]$ gewählt, so kann dann das Paar $\left[r_{2}\right],\left[s_{2}\right]$ nach $\mathrm{XVI}$. ebenso anf

$$
\frac{\left(2^{2(p-1)}-1\right) 2^{2(p-1)-1}}{2}=\frac{1}{2} R_{2(p-1)}
$$

Weisen bestimmt werden; alsdann $\left[r_{3}\right],\left[s_{3}\right]$ auf $\frac{1}{2} R_{2(p-2)}$ Weisen, etc. Endlich kann man noch die $\mu$ Paare willkürlich permutiren, ohne die gegenseitigen Beziehungen der Paare des Systems, sowie die in den Gruppen des Systems enthaltenen Charakteristiken $(a)$ und (b) zu ändern. Dies giebt die Zahl $\boldsymbol{M}_{\boldsymbol{\mu}}$.

Die Zahl $N_{\mu}$ folgt aus XIX. Sind nämlich die $(a)$ und $(b)$ gegeben und identiscls mit den in XVI. so bezeichneten, so kann man 
für die erste Gruppe $\left[r_{1}\right]$ irgend eine der $S_{\mu}-1$ Gruppeu [u] uehmen; z. B. $\left[r_{1}\right]$ selbst; für die zweite Gruppe $\left[s_{.}\right]$alsdann irgend eine der Gruppen $[u]$, welche mit der gewählten $\left[r_{i}\right]$ die Beziehung $K_{r_{1}, n} \equiv 1$ haben, also eine der folgenden

$$
\left[s_{1}\right],\left[s_{1} u_{1}\right],\left[r_{1} s_{1} v_{1}\right],
$$

wenn man unter $\left[u_{1}\right]$ und $\left[v_{1}\right]$ die aus den $\mu-1$ Paarel

$$
\left[r_{2}\right],\left[s_{2}\right] ; \cdots\left[r_{\mu}\right],\left[s_{\mu}\right]
$$

von XVI. zusaumengesetzten Gruppen $[u]$, bez. $[v]$, versteht. D. h. $\left[s_{1}\right]$ kaun noch auf $1+\left(S_{\mu-1} 1\right)+R_{\mu-2}=2^{2(\mu-1)}$ Weisen gewählt werden. Man kann dann wieder weiter die beiden Gruppen des ersten Paares mit einander vertausehen, also das erste Paar auf $\left(S_{\mu}-1\right) \cdot 2^{2 \mu-9}$ $=R_{\mu} S_{\mu-1}$ Weisen wählen, das zweite Paar analog dem ersten auf

$$
\left(S_{\mu-1}-1\right) 2^{2(\mu-2)-1}=R_{\mu-1} S_{\mu-2}
$$

Weisen, dieses mit dem ersten Paar vertauschen, etc.; was, mit Hülfe der Relation $2 R_{\mu} S_{\mu}=R_{2 \mu}, N_{\mu}$ liefert.

Der letzte Theil des Satzes ist evident.

3. Dit Eintheilung der Gruppen in Systeme nach XVI. liefert auch sogleich eine Gruppirung der Substitutionen, welche die Charakteristikenbeziehungen unverändert lassen.

Seien die sämmtlichen Substitutionen mit

$$
A, B, C, \cdots
$$

bezeichnet, und ihre Anzabl sei $Z$. Diejenigen derselben, welche die beiden Gruppen des Paares $\left[r_{1}\right],\left[s_{1}\right]$ in sicb überführen, seien nit

$$
A_{1}, B_{1}, C_{1}, \cdots \text {, }
$$

und deren Anzahl mit $Z_{1}$ bezeichnet. Nach XI., \& 3., kann man das $\operatorname{Paar}\left[r_{1}\right],\left[s_{1}\right]$ in jedes andere Paar $\left[r_{1}^{\prime}\right],\left[s_{1}^{\prime}\right]$, für das $K_{r_{r}, r_{1}^{\prime}} \equiv 1$, durch eine Substitution überführen, und zwar $\left[r_{1}\right]$ beliebig in $\left[r_{1}^{\prime}\right]$ oder in $\left[s_{1}^{\prime}\right] \cdot\left[r_{1}^{\prime}\right],\left[s_{1}{ }^{\prime}\right]$ sind dabei (s. $2 . \& 5-j$ auf $R_{2 p}$ verschiedene Weisen wählbar, und jeder einzelnen entsprechend sei eine bestimmte, im Uebrigen beliebige, Substitution ausgewählt, welche $\left[r_{1}\right]$ in $\left[r_{1}^{\prime}\right],\left[s_{1}\right]$ in [ $\left.s_{1}^{\prime}\right]$ überfübrt. Diese $R_{2 p}$ bestimmten Substitutionen seien mit

$$
\mathrm{A}, \mathrm{B}, \Gamma, \cdots
$$

bezeichnet. Die Substitutionen $A, B, C, \ldots$ sind dann das Product der Substitutionen $A_{1}, B_{1}, C_{1}, \cdots$ und $\mathrm{A}, \mathrm{B}, \Gamma, \cdots$, also:

XXIl. Dic sümmtlichen Sulstitutionen $A, B, C, \cdots$ sind in der Form darstelluar:

$$
\begin{aligned}
& A_{1} \mathrm{~A}, \quad B_{1} \mathrm{~A}, \quad C_{1} \mathrm{~A}, \cdots \text {, } \\
& A_{1} \mathrm{~B}, \quad B_{1} \mathrm{~B}, C_{1} \mathrm{~B}, \cdots \text {, } \\
& A_{1} \Gamma, \quad B_{1} \beta, \quad C_{1} \Gamma, \cdots \text {, }
\end{aligned}
$$


und ihre Anzahl beträgt

$$
Z=R_{2 p} \cdot Z_{1}
$$

Auf dieselbe Weise kann man weiter die

$$
A_{1}, B_{1}, C_{1}, \cdots
$$

darstellen als das Product derjenigen $Z_{2}$ Substitutionen

$$
A_{2}, B_{2}, C_{2}, \cdots
$$

welche die Gruppen eines zweiten Paares von XVI., \& 4., $\left[r_{2}\right],\left[s_{2}\right]$, in sich überführen, mit solehen $R_{2(p-1)}$ Substitutionen,

$$
A_{1}, B_{1}, r_{1}, \cdots \text {, }
$$

durch welche $\left[r_{2}\right],\left[s_{2}\right]$ in jedes Paar $\left[r_{2}^{\prime}\right],\left[s_{2}^{\prime}\right]$ übergehen, für welches:

$$
K_{r^{\prime}, s_{2}^{\prime}} \equiv 1, \quad K_{r_{2}^{\prime}, r_{1}} \equiv K_{r_{2}^{\prime}, s_{1}} \equiv K_{s_{2}^{\prime}, r_{1}} \equiv K_{s_{i}^{\prime}, s_{t}} \equiv 0 \text {. }
$$

Und indem man so fortfährt, kann man endlich die $Z_{p-2}$ Substitutionen, welche die Gruppen der Art XVI.:

$$
\left[r_{1}\right],\left[s_{1}\right] ;\left[r_{2}\right],\left[s_{2}\right] ; \cdots\left[r_{p-2}\right],\left[s_{p-2}\right]
$$

unverändert lassen, darstellen als das Product derjenigen $Z_{p-1}$ Substitutionen, welche unter jenen enthalten sind und zugleich ein weiteres Paar

$$
\left[r_{p-1}\right],\left[s_{p-1}\right]
$$

in sich überführen, mit den $R_{4}$ Substitutionen, welche dieses Paar in jedes seiner in XVI. gegebenen Definition entsprechende Paar überführen. Aber die $Z_{p-1}$ Substitutionen sind aus denjenigen 3 geraden Charakteristiken $\left(a_{1}\right),\left(a_{2}\right),\left(a_{3}\right)$ zusammenzusetzen, welche in den Gruppen

$$
\left[r_{1}\right],\left[s_{1}\right] ; \cdots ;\left[r_{p-1}\right],\left[s_{p-1}\right]
$$

enthalten sind, und welche diese 3 Grössen beliebig in einander überführen, was

$$
Z_{p-1}=6=R_{2}
$$

liefert. Daher hat mau als Ergänzung von XXII.:

XXII'. Die Anzahl aller Substitutionen $A, B, C, \ldots$ beträgt:

$$
Z=R_{2 p} \cdot R_{2(p-1)} \cdots R_{4} \cdot R_{2} \text {. }
$$

\section{$\S 6$.}

\section{Charakteristikensysteme.}

1. Nach den in \& 1 . gegebenen Erläuterungen werden Charakteristikensysteme mit besonderen Higenschaften solche sein, für welche die Summe irgend einer ungeraden Zahl ihrer Charakteristiken einen gegebenen Charakter (des Geraden oder Ungeraden) hat. Zwischen diesen Summen existiren aber Beziehungen. 
Sei nämlich erstens ein System von $N$ geraden Charakteristiken:

$$
\left(a_{1}\right),\left(a_{2}\right), \cdots,\left(a_{N}\right)
$$

gegeben, für welches, für alle von einander verschiedenen $\iota, x, \lambda$

$$
\left(a_{t} a_{x} a_{2}\right)
$$

ungerade ist. Zunächst folgt hieraus, dass alle

$$
\left(a_{\iota} a_{x} a_{2} a_{\mu} a_{\nu}\right) \quad \text { (wo } \iota \gtrless \varkappa \gtrless \lambda \gtrless \mu \gtrless \nu \text { ) }
$$

gerade sind. Denn die beiden Gruppen

stehen in der Beziehung

$$
\left[a_{4} a_{x}\right], \quad\left[a_{2} a_{\mu}\right]
$$

$$
\boldsymbol{K}_{a_{1} a_{x}, a_{2} a_{\mu}} \equiv 0 \text {, }
$$

da von den beiden in $\left[a_{t} a_{x}\right]$ euthaltenen gepaarten Charakteristiken $\left(a_{t}\right),\left(a_{x}\right)$, wegen $\left(a_{2} a_{k} a_{z}\right)$ und $\left(a_{\lambda} a_{\mu} a_{x}\right)$ ungerade, keine in $\left[a_{2} a_{\mu}\right]$ vorkommt; eine Charakteristik $\left(a_{v}\right)$ also, die in $\left[a_{\imath} a_{x}\right]$ und in $\left[a_{i} a_{\mu}\right]$ nicht enthalten ist, muss nach VII., \$ 2., in der durch Summirung dieser beiden Gruppen entstehenden Gruppe $\left[a_{4} a_{x} a_{2} a_{\mu}\right]$ enthalten sein; d. h. $\left(a_{t} a_{x} a_{2} a_{4} a_{v}\right)$ wird gerade.

Hieraus folgt dann weiter, dass alle aus 7 verschiedenen $\left(a_{i}\right) \mathbf{z u}$ sammengesetzten Charakteristiken ungerade sind. Denn zwei Gruppen

$$
\left[a_{\imath} a_{x}\right], \quad\left[a_{2} a_{\mu} a_{v} a_{\mathrm{g}}\right]
$$

haben ebenfalls die Beziehung $K \equiv 0$, da $\left(a_{i}\right),\left(a_{k}\right)$ in beiden Gruppen, und in der ersten gepaart, vorkommen; von dem Paar der zweiten Gruppe

$$
\left(a_{\sigma}\right), \quad\left(a_{2} a_{\mu} a_{\nu} a_{\mathrm{e}} a_{\sigma}\right)
$$

ist daher, wie $\left(a_{\sigma}\right)$ nicht, so auch $\left(a_{\lambda} a_{\mu} a_{v} a_{\rho} a_{a}\right)$ nicht in der Gruppe $\left[a_{\iota} a_{x}\right]$ enthalten; d. h. $\left(a_{t} a_{x} a_{2} a_{4} a_{2} a_{q} a_{\sigma}\right)$ wird ungerade, wenn $\iota, x, \lambda \ldots$ von einander verschieden sind.

Diese Schlüsse lassen sich auf Summen von $9,11, \ldots$ Charakteristiken $\left(a_{i}\right)$ analog fortsetzen; und es wird die Summe von $4 n+1$ verschiedenen Charakteristiken $\left(a_{i}\right)$ inmer gerade, die von $4 n+3$ ungerade.

Sei zweitens gegeben ein System von $N$ geraden Charakteristiken:

$$
\left(c_{1}\right) ;\left(c_{2}\right), \cdots,\left(c_{N}\right),
$$

und eine gerade Charakteristik (c), für welche

$$
\left(c c_{\imath} c_{x}\right)
$$

ungerade ist, für alle $\iota \lessgtr x$. Hieraus folgt, dass alle

$$
\left(c_{t} c_{x} c_{2}\right)
$$

ungerade, und dass alle 
gerade sind. Denn die beiden Gruppen

$$
\left[c c_{i}\right], \quad\left[c_{x} c_{2}\right]
$$

stehen in der Beziehung $K \equiv 0$, weil die in $\left[c_{x} c_{2}\right]$ gepaarten $\left(c_{x}\right),\left(c_{2}\right)$ beide nicht in $\left[c c_{z}\right]$ enthalten sind. Die Charakteristik $(c)$, die in $\left[c c_{t}\right]$ enthalten, in $\left[c_{x} c_{2}\right]$ nicht enthalten ist, kann daher auch in der Summe der beiden Gruppen $\left[c c_{2} c_{z} c_{2}\right]$ nach VII. nicht enthalten sein, d. h. $\left(c_{2} c_{x} c_{\lambda}\right)$ ist ungerade. Ferner ist $\left(c_{\mu}\right)$ weder in $\left[e c_{\ell}\right]$, noch in $\left[c_{x} c_{\lambda}\right]$ enthalten, ist daher in $\left[c c_{2} c_{x} c_{2}\right]$ enthalten; d. h. $\left(c c_{t} c_{x} c_{2} c_{\mu}\right)$ ist gerade. Man schliesst hieraus weiter, dass $\left(c c_{1} c_{x} c_{2} c_{u} c_{r} c_{\varrho}\right)$ ungerade wird, etc.

Wäre hier $(c)$ ungerade und alle $\left(c c_{1} c_{k}\right)$ gerade, so folgte ganz ebenso, dass alle $\left(c c_{t} c_{x} c_{2} c_{\mu}\right)$ ungerade, die $\left(c c_{t} c_{x} c_{\lambda} c_{\mu} c_{\nu} c_{Q}\right)$ gerade etc. würden, und alle $\left(c_{t} c_{x} c_{2}\right)$ ungerade.

Insbesondere kann, wenn $N$ ungerade ist, für $(c)$ die Summe

$$
\left(c_{1} c_{2} \cdots c_{N}\right)
$$

gegeben sein, und zwar (c) gerade, wenn $N$ von der Form $4 n+1$, ungerade, wenn $N$ von der Form $4 n+3$.

Genau dieselben Schlüsse, mit Vertauschung von Gerade und Ungerade, finden statt, wenn alle $a_{\iota}$ oder $c_{\iota}$ ungerade sind. Man hat also den Satz:

XXIII. Damit in einem System von $N$ geraden [ungeradcn] Charakteristiken die Summe von irgend $4 n+1$ derselben gerade [ungerade], die Summe von irgend $4 n+3$ derselben ungerade [gerade] werde, ist die nothwendige und hinreichende Bedingning die, dass dic Summe je dreier der Charakteristiken ungerade [gerade] werde. Statt dessen genügt auch, dass eine Charakteristik (c) existire, welche mit je zweien der yegebenen Charalteristiken verbunden, inmer eine ungerade Summe, wenn (c) gerade ist, oder eine gerade Summe, wenn (c) ungerade ist, liefert. In diesem Falle giebt dunn auch (c), mit irgend $4 n$ der Charakteristiken verbunden, eine Summe van demselben Charahter des Geraden oder Ungeraden, wie (c); mit $4 n+2$ der Charakteristiken verbunden, eine Summe von entgegengesetztem Charalter. Wenn $N$ ungerade, so muss die Summe der $N$ Charahteristiken eine solche Charakteristik $(c)$ sein.

2. XXIV. In einem System von $2 \pi+1$ geraden [ungeraden] Charakteristiken $\left(c_{t}\right)$, für welches die Summe je dreier ungerade [gerade] ist, sind die Combinationen derselben $z u 1,3,5, \cdots, 2 \pi+1$, ebenso die von $2,4, \cdots, 2 \pi$, alle von einander verschieden. Die Combinationen $z u 1,5,9, \ldots$ liefern $S_{\pi}$ Charakteristiken, wenn $\pi$ von der Form $4 n$ oder $4 n+1$, aber $h_{n}$, wenn $\pi$ von der Form $4 n+2$ oder $4 n+3$; die Combinationen zu $3,7,11, \ldots$ liefern in diesen Fällen $R_{\pi}$ bes. $S_{n}$, Charakteristiken. Die Combinationen zu 2, 6, 10, . liefern $R_{\pi}$ Gruppen, wenn $\pi$ von der Form $4 n$ oder $4 n+3$, aber $S_{\pi}$, wenn $\pi$ 
von der Form $4 n+1$ oder $4 n+2$; die Combinationen $z u 4,8,12, \ldots$ liefern in diesen Fällen $S_{\pi}-1$, bez. $R_{\pi}-1$ Gruppen. Diese $2 \geq z-1$ Gruppen setzen sich schon aus den $2 \pi+1$ Gruppon $\left[c c_{t}\right]$, deren Summe verschwindet, zusammen, wo (c) die Summe der $\left(c_{i}\right)$ vorstellt; die Charakteristiken nur aus den $2 \pi+1$ Grössen $\left(c_{1}\right)$ mit nicht verschwindender Summe.

Denn wären zwei solche Combinationen einander gleich, so würde dereu Summe, ein aus einer geraden Anzahl der Charakteristiken zusammengesetzter Ausdruck, zu 0:

$$
\left[c_{1} c_{2} \cdots c_{z_{u-1}} c_{z_{k}}\right\}=0 \text {. }
$$

Weun aber $\mu$ von der Form $2 v$, kann diese Gleichung nicht bestehen, weil $\left(c_{2 \mu}\right)$ und $\left(c_{1} c_{2} \cdots c_{2 \mu-1}\right)$ von entgegengesetztem Charakter sind; und wenn $\mu$ von der form $2 v+1$, ebenfalls nicht, weil, unter $\left(c_{z \mu+1}\right)$ eine weitere von $\left(c_{1}\right), \cdots\left(c_{2 \mu}\right)$ verschiedene Charakteristik des Systems verstanden, $\left(c_{2 \mu+1}\right)$ und $\left(c_{1} c_{2} \cdots c_{2 \mu} c_{z \mu+1}\right)$ von entgegengesetztem Charakter sind. - Die in dem Satze gegebenen Anzahlen bestimmen sich hiernach durch einfache Abzählung (am einfachsten durch den Selhluss von $\pi-1$ auf $\pi$ ).

3. Nach dem in 2. gegebenen Satz XXIV. wird man, um alle $S_{p \sim \mu}$ geraden und $R_{p-\mu}$ ungeraden Charakteristiken, $(a)$ bez. $(b)$, zu erhalten, welche in den $2 \mu$ Gruppen

$$
\left[r_{1}\right],\left[s_{1}\right] ;\left[r_{2}\right],\left[s_{2}\right] ; \cdots ;\left[r_{\mu}\right],\left[s_{\mu}\right]
$$

des Satzes XVI., \& 4., enthalten sind, ein System von $2(p-\mu)+1$ dieser Charakteristiken $(a)$ bilden, für welches die Summe je dreier ungerade; oder ein System von $2(p-\mu)+1$ der $(b)$, für welches die Summe je dreier gerade ist; und zwar aus den $(a)$ im Falle $p-\mu$ von der Form $4 n$ oder $4 n+1$, aus den $(b)$ im Falle $p-\mu$ von der Form $4 n+2$ oder $4 n+3$. Die Combinationen aus den $2(p-\mu)+1$ Grössen eines Systems liefern dann nämlich nach XVIII., §4., nur wieder Charakteristiken $(a),(b)$ und Gruppen $\left[r_{\mu+1}\right] ;$ und zwar nach. XXIV. jede dieser Charahteristiken und Gruppen, und jede nur eimmal.

Dabei ist zu bemerken, dass sich die $2^{2(p-\mu)}-1$ Gruppen schon aus deu Combinationen von $2(p-\mu)$ solchen zusammensetzen, nämlich aus den $\left[c c_{l}\right]$, deren Summe $=0$ ist, wenn das System mit

$$
(c) \equiv\left(c_{1}\right),\left(c_{z}\right), \cdots\left(c_{2 p-2,4+1}\right)
$$

bezeichnet wird; dass aber die eigentlichen Charakteristiken erst aus den $2(p-\mu)+1$ Grössen $\left(c_{t}\right)$ combiuirt werden können, deren Summe nicht verschwindet.

Der Satz XX., \& 4., ergiebt endlich eine Darstellung für sämmtliche $2^{2 p}$ Charakteristiken und $2^{* p}-1$ Gruppen. 


\section{$\S 7$.}

\section{Die Systeme von $2 \pi+1$ Charakteristiken.}

Wir beweisen in diesem Paragraphen die Existenz der in $3 ., \S 6$. bezeichneten Systeme von $2(p-\mu)+1$ Charakteristiken, für $\mu=p$, $p-1, \cdots, 0$, zugleich mit dem Gesetz ihrer Bildung.

1. Die $2 p$ Gruppen (wobei $r_{i} s_{i}=t_{i}$ gesetzt werde):

$$
\left[r_{1}\right],\left[s_{1}\right] ;\left[r_{\mathrm{s}}\right],\left[s_{2}\right] ; \cdots ;\left[r_{p}\right],\left[s_{p}\right] \text {, }
$$

welche in den Beziehungen

$$
K_{r_{i}, s_{i}} \equiv 1, \quad K_{r_{i}, r_{k}} \equiv K_{r_{i}, s_{k}} \equiv K_{s_{i}, s_{k}} \equiv 0
$$

stehen, haben, nach XVI., eine gerade Charakteristik gemein, die mit $(g)$ bezeichnet sei. ( $g$ ) repräsentirt für sich eines der gesuchten Systeme, für $\mu=p$.

Hieraus kann man ein System herstellen:

oder abgekürzt:

$$
\left(g_{1}\right)=\left(g t_{p}\right) \equiv(g), \quad\left(g r_{p}\right),\left(g s_{p}\right),
$$

$$
\left(g_{1}\right) \equiv\left(g_{11}\right),\left(g_{12}\right), \quad\left(g_{13}\right)
$$

bestehend aus 3 geraden Charakteristiken

$$
\left(g_{11}\right)=(g), \quad\left(g_{12}\right)=\left(g r_{p}\right), \quad\left(g_{13}\right)=\left(g s_{p}\right),
$$

welche, nach XIX., alle in den 2(p-1) Gruppen

$$
\left[r_{1}\right],\left[s_{1}\right] ;\left[r_{2}\right],\left[s_{2}\right] ; \cdots ;\left[r_{p-1}\right],\left[s_{p-1}\right]
$$

enthalten sind, und deren Summe $\left(g t_{p}\right)=\left(g_{1}\right)$ ungerade ist. Dieses ist also eines der gesuchten Systeme, und zwar für $\mu=p-1$. Die sämmtlichen, in den $2(p-1)$ Gruppen enthaltenen Charakteristiken sind in der That:

$$
(g),\left(g r_{p}\right),\left(g s_{p}\right),\left(g t_{p}\right) .
$$

Um aus dem System $\left(g_{1}\right)$ weiter ein System von 5 ungeraden Charakteristiken, für $\mu=p-2$, herzustellen, setzen wir jede der Charakteristiken von $\left(g_{1}\right)$ mit $t_{p-1}$ zusammen, und fügen $\left(g_{1} r_{p-1}\right)$, $\left(g_{1} s_{p-1}\right)$ hinzu. Dies ergiebt das System:

$$
\left(g_{2}\right)=\left(g_{1}\right) \equiv\left(g_{11} t_{p-1}\right), \quad\left(g_{12} t_{p-1}\right), \quad\left(g_{13} t_{p-1}\right), \quad\left(g_{1} r_{p-1}\right), \quad\left(g_{1} s_{p-1}\right),
$$

oder abgekürzt:

$$
\left(g_{2}\right) \equiv\left(g_{21}\right),\left(g_{22}\right),\left(g_{23}\right),\left(g_{24}\right),\left(g_{25}\right),
$$

bestehend aus 5 ungeraden Charakteristiken $\left(g_{2}\right)$, welche alle in deu $2(p-2)$ Gruppen

$$
\left[r_{1}\right],\left[s_{1}\right] ; \cdots ;\left[r_{p-2}\right],\left[s_{p-2}\right]
$$

enthalten sind, und deren Combinationen zu 3 gerade, zu 5 ungerade sind. 
Aus dem System $\left(g_{2}\right)$ ergiebt sich weiter ein System für $\mu=p-3$, wenn man die Charakteristiken von $\left(g_{3}\right)$ unverändert lässt und nur $\left(g_{2} r_{p-2}\right),\left(g_{2} s_{p-2}\right)$ hinzufügt:

$$
\left(g_{2} t_{p-2}\right) \equiv\left(g_{21}\right),\left(g_{22}\right), \cdots,\left(g_{35}\right),\left(g_{2} r_{p-2}\right),\left(g_{12} s_{p-2}\right)
$$

oder abgekürıt:

$$
\left(g_{3}\right) \equiv\left(g_{31}\right),\left(g_{32}\right), \cdots,\left(g_{37}\right),
$$

bestehend aus 7 ungeraden Charakteristiken $\left(g_{3}\right)$, welche alle in den $2(p-3)$ Gruppen

$$
\left[r_{1}\right],\left[s_{1}\right] ; \cdots ;\left[r_{p-3}\right],\left[s_{p-s}\right]
$$

enthalten sind, und deren Combinationen zu 3 und 7 gerade, zu 5 ungerade sind.

Aus dem System $\left(g_{3}\right)$ folgt ein System $\left(g_{4}\right)$, für $\mu=p-4$ :

$$
\left(g_{4}\right)=\left(g_{3}\right) \equiv\left(g_{31} t_{p-s}\right),\left(g_{32} t_{p-3}\right), \cdots,\left(g_{37} t_{p-3}\right),\left(g_{3} r_{p-s}\right),\left(g_{3} \dot{s}_{p-s}\right) \text {, }
$$

von den in $\$ 6$. . 3. angezeigten Eigenschaften.

Aualog ergeben sich sodann die Systeme für $\mu=p-4 n-1$, $p-4 n-2, p-4 n-3, p-4 n-4$ aus einem System für $\mu=p-4 n$. Sei in der That

$$
(c)=\left(c_{1}\right),\left(c_{2}\right), \cdots,\left(c_{2(p-\mu-1)+1}\right)
$$

irgend ein System von $2(p-\mu)-1$ Charakteristiken, gebildet aus den in den $2(\mu+1)$ Gruppen

$$
\left[r_{1}\right],\left[s_{1}\right] ; \cdots ;\left[r_{\mu}\right],\left[s_{\mu}\right] ;\left[r_{\mu+1}\right],\left[s_{\mu+1}\right]
$$

(von den in $X V I$. angegebenen Beziehungen) enthaltenen Charakteristiken; wobei für $p-\mu-1=4 n$ oder $4 n+1$ die $\left(c_{t}\right)$ gerade, $\left(c_{4} c_{x} c_{2}\right)$ ungerade, für $p-\mu-1=4 n+2$ oder $4 n+3$ aber die $\left(c_{t}\right)$ ungerade, $\left(c_{t} c_{x} c_{2}\right)$ gerade sein sollen.

Sei nun erstens $p-\mu-1$ gerade: dann hat man in

$$
\left(c t_{\mu+1}\right) \equiv\left(c_{1}\right),\left(c_{2}\right), \cdots,\left(c_{2(p-\mu-1)+1}\right),\left(c r_{\mu+1}\right),\left(c s_{\mu+1}\right)
$$

ein System von $2(p-\mu)+1$ Charakteristiken der gesuchten Art. Dieses sind nämlich, ftr $p-\mu-1=4 n[4 n+2], 2(p-\mu)+1$ gerade [ungerade] Charakteristiken, für welche die Summe je dreier ungerade [gerade] ist, da nach XXIII. die $\left(c c_{1} c_{x}\right)$ ungerade [gerade] sind, also auch nach XIX., die

$$
\left(e r_{\mu+1} c_{l} c_{x}\right),\left(c s_{\mu+1} c_{t} c_{x}\right),\left(t_{\mu+1} c_{l}\right),
$$

und welche in den Groppen

enthalten sind.

$$
\left[r_{1}\right],\left[s_{1}\right] ; \cdots ;\left[r_{\mu}\right],\left[s_{\mu}\right]
$$

Sei zweitens $p-\mu-1$ ungerade: daun wird

$$
(c) \equiv\left(c_{1} t_{\mu+1}\right),\left(c_{2} t_{\mu+1}\right), \cdots,\left(c_{2(p-\mu-1)+1} t_{\mu+1}\right),\left(c r_{\mu+1}\right),\left(c s_{\mu+1}\right)
$$


ein System der gesuchten Art. Denn man hat hier, für $p-\mu-1$ $=4 n+1[4 n+3] 2(p-\mu)+1$ ungerade [gerade] Charakteristiken, welche in den Gruppen

$$
\left[r_{1}\right],\left[s_{1}\right] ; \cdots ;\left[r_{\mu}\right],\left[s_{\mu}\right]
$$

enthalten sind, und für welche die Summe je dreier gerade [ungerade] ist, da nach XXIII. und XIX. die

also auch die

$$
\left(c_{1} c_{x} c_{2} t_{u+1}\right), \quad\left(c c_{1} c_{x}\right)
$$

$$
\left(c c_{\imath} c_{\mathbf{x}} r_{\mu+1}\right), \quad\left(c c_{\iota} c_{\boldsymbol{x}} s_{\mu+1}\right)
$$

gerade [ungerade] werden.

2. Wir können jetzt zeigen, dass auch umgekehrt jedes System der Art 3. $\S 6$., gebildet aus $2(p-\mu)+1$ Charakteristiken, die in den $\boldsymbol{\mu}$ Gruppenpaaren der Art XVI., $\left[r_{i}\right],\left[s_{i}\right]$, enthalten sind, auf die in Nr. 1. dieses Paragraphen gegebene Weise aus einem solchen System von $2(p-\mu)-1$ Charakteristiken, die in $\mu+1$ Gruppenpaaren der Art XVI. enthalten sind, abgeleitet werden kann.

Seien die $\mu$ Gruppenpaare

$$
\left[r_{1}\right],\left[s_{1}\right] ; \cdots ;\left[r_{\mu}\right],\left[s_{\mu}\right],
$$

und das System, gebildet aus in diesen Gruppen enthaltenen Charakteristiken:

Die beiden Gruppen

$$
(d) \equiv\left(d_{1}\right),\left(d_{2}\right), \cdots,\left(d_{2(p-\mu)+1}\right) .
$$

$$
\left[d d_{2(p-\mu)}\right]=\left[r_{\mu+1}\right], \quad\left[d d_{2(p-\mu)+1}\right]=\left[s_{\mu+1}\right]
$$

gehören zunächst zu den in XVI. definirten Gruppen $\left[r_{\mu+1}\right]$, welche zu deu obigen $2 \mu$ Gruppen die Beziehung $K \equiv 0$ haben (nach XVIII.). Ferner ist $K_{r_{\mu+1}, s_{\mu+1}}=1$, da $(d)$ und $\left(d d_{2(\gamma-\mu)} d_{2(p-\mu)+1}\right)$ von ungleichem Charakter sind, also von der Zerlegung der Gruppe

$$
\begin{gathered}
{\left[r_{\mu+1} s_{\mu+1}\right]=\left[t_{\mu+1}\right],} \\
{\left[t_{\mu+1}\right] \equiv\left(d_{2(p-\mu)}\right), \quad\left(d_{2(p-\mu)+1}\right)}
\end{gathered}
$$

nur eine der beiden Charakteristiken in $\left[r_{\mu+1}\right]$ entbalten ist. Man hat daher $\mu+1$ Gruppenpaare der Art XVI.:

$$
\left[r_{1}\right],\left[s_{1}\right] ; \cdots ;\left[r_{\mu}\right],\left[s_{\mu}\right] ;\left[r_{\mu+1}\right],\left[s_{\mu+1}\right] .
$$

Ist nun erstens $p-\mu$ ungerade, so sind

$$
\left(d t_{\mu+1}\right)=-\left(d_{1}\right),\left(d_{2}\right), \cdots,\left(d_{2(p-\mu-1)+1}\right)
$$

alle in $\left[r_{\mu+1}\right]$ und in $\left[s_{\mu+1}\right]$ enthalten, da dann $\left(d d_{\iota} d_{x}\right)$ mit $\left(d_{\varepsilon}\right)$ zugleich gerade oder zugleich uugerade sind. Diese $2(p-\mu)-1$ Charakteristiken bilden das gresuchte System, enthalten in den obigen $\mu+1$ Gruppenpaaren. 
Ist zweitens $p-\mu$ gerade, so sind

$$
(d) \equiv\left(d_{1} t_{\mu+1}\right),\left(d_{2} t_{\mu+1}\right), \cdots,\left(d_{2(p-\mu-1)+1} t_{\mu+1}\right)
$$

alle in $\left[r_{\mu+1}\right]$ und in $\left[s_{\mu+1}\right]$ enthalten, da dann $\left(d d_{t} d_{x}\right)$ und $\left(d_{t} d_{x} d_{2}\right)$ zugleich gerade oder zugleich ungerade sind. Diese $2(p-\mu)-1$ Charakteristiken bilden alsdann das verlangte System, welches in den obigen $\mu+1$ Gruppenpaaren enthalten ist.

3. Man kann endlich zeigen, das jedes System von $2(p-\mu)+1$ Charakteristiken, welche für $p-\mu=4 n$ oder $4 n+1$ gerade, für $p-\mu=4 n+2$ oder $4 n+3$ ungerade sind, und für welche die Summe je dreier ungerade, bez. gerade ist, zugleich eines der in diesem Paragraphen betrachteten ist; $d . h$. dass jedes solche System ans Charakteristiken besteht, welche in $\mu$ Gruppenpaaren der Art XVT. euthalten sind.

Für $\mu=p$ ist dieses der Fall. Wir nehmen daher an, dass die Behauptung für die Systene von $2(p-\mu)-1$ Charakteristiken richtig ist, um sie hieraus für die von $2(p-\mu)+1$ zu beweisen.

Sei das System ron $2(p-\mu)+1$ Charakteristiken:

$$
(d)=\left(d_{1}\right),\left(d_{2}\right), \because,\left(d_{2(p-\mu)+1}\right) ;
$$

so folgt zunächst, wie in 2. dieses Paragraphen, dass die beiden Gruppen

$$
\left[d d_{2(\mu-\mu)}\right]=\left[r_{\mu+1}\right], \quad\left[d d_{2(p-\mu)+1}\right]=\left[s_{\mu+1}\right]
$$

in der Beziehung $K_{r_{\mu+3}, s_{\mu+1}} \equiv 1$ stehen.

Ist sodann wieder erstens $p-\mu$ ungerade, so sind die Charakteristiken des Systems

$$
\left(d t_{\mu}\right) \equiv\left(d_{1}\right),\left(d_{3}\right), \cdots,\left(d_{s(p-\mu)-1}\right),
$$

sowie deren Combinationen zu $3,5, \ldots$ alle in $\left[r_{\mu+1}\right]$ und in $\left[s_{\mu+1}\right]$ enthalten. Für dieses System gilt aber die obige Annahme; es existiren also $\mu+1$ Gruppenpaare, von der Art XVI., welche alle $S_{p-\mu-1}$ geraden und $R_{p-\mu-1}$ ungeraden Charakteristiken, die durch diese Combinationen entstehen, enthalten. Und für eines dieser Paare kann (vgl. die Bestimmung der Zahl $N_{\mu}$ in Satz XXI.) $\left[r_{\mu}\right] .\left[s_{\mu}\right]$ genommen werden. Die äbrigen Gruppenpaare sein:

$$
\left[r_{1}\right],\left[s_{1}\right] ;\left[r_{2}\right],\left[s_{2}\right] ; \cdots ;\left[r_{\mu}\right],\left[s_{\mu}\right],
$$

wo

$$
K_{r_{\mu+1}, r_{2}} \equiv K_{s_{\mu+1}, r_{1}} \equiv K_{r_{\mu+1}, r_{2}} \equiv \cdots=K_{s_{\mu+1}, s_{\mu}} \equiv 0 \text {. }
$$

Es bleilit dann nur noch ïbrig zn zeiren, dass, ebenso wie

$$
\left(d_{1}\right),\left(d_{2}\right), \cdots,\left(d_{2 i p-\mu ;-1}\right),
$$

so auch $\left(d_{2(p-\mu)}\right)$ und $\left(d_{2(p-\mu)+1}\right)$ in jeder dieser $2 \mu$ Gruppen enthalten sind. 
Nun stehen aber die beiden Gruppen $\left[r_{1}\right]$ und

$$
\left[s_{\mu+1}\right] \equiv\left(d_{2(p-\mu)}\right),\left(d t_{\mu+1}\right)
$$

in der Beziehung $K_{r_{1}, s_{\mu+1}} \equiv 0$; und da $\left[r_{1}\right]$ die Charakteristik $\left(d t_{\mu+1}\right)$, die Summe der objgen $2(p-\mu)-1$ Charakteristiken, enthält, so muss auch die in $\left[s_{\mu+1}\right]$ damit gepaarte Charakteristik $\left(d_{2(p-\mu)}\right)$ in $\left[r_{1}\right]$ vorkommen; ebenso in $\left[s_{1}\right]$, etc. Dasselbe gilt für $\left(d_{2(p-\mu)+1}\right)$.

Ist zweitens $p-\mu$ gerade, so ist der analoge Schluss auf das System

$$
(d) \equiv\left(d_{1} t_{\mu+1}\right),\left(d_{2} t_{\mu+1}\right), \cdots,\left(d_{2(p-\mu)-1} t_{\mu+1}\right)
$$

anzuwenden. Da nämlich, wenn $\left[r_{1}\right]$ wie oben bestimmt ist, $\left(d_{1} t_{\mu+1}\right)$ in $\left[r_{1}\right]$ enthalten ist, so hat man:

$$
\begin{aligned}
\sum\left(n^{d_{1} r_{1}} m^{d_{1} r_{1}}+n^{d_{1}} m^{d_{1}}\right) & \equiv \sum\left(n^{d_{1} r_{1} t_{\mu+1}} m^{d_{1} r_{1} t_{\mu+1}}+n^{d_{1} t_{\mu+1}} m^{d_{1} s_{\mu+1}}\right) \\
& +\sum\left(n^{r_{1}} m^{t_{\mu+1}}+n^{t_{\mu+1}} m^{r_{1}}\right) \equiv 0,
\end{aligned}
$$

d. h. $\left(d_{1}\right)$ ist ebenfalls in $\left[r_{1}\right]$ enthalten. Für $\left(d_{2(p-\mu)}\right)$ folgt dasselbe ähnlich wie oben.

4. Die Resultate dieses Paragraphen lassen sich so aussprechen:

XXV. In jedem System von $2(p-\mu)+1$ Charalteristiken, deren Combinationen

für $p-\mu=4 n$ oder $4 n+1 \quad\left\{\begin{array}{l}z u 1,5,9, \cdots \text { gerade Summen, } \\ z u 3,7,11, \cdots \text { ungerade Summen, }\end{array}\right.$ für $p-\mu=4 n+2$ oder $4 n+3\left\{\begin{array}{l}z u 1,5,9, \ldots \text { ungerade Summen, } \\ z u 3,7,11, \ldots \text { gerade Summen }\end{array}\right.$ liefern (wo $\mu=0,1, \ldots$, oder $p$ sein liann), wozu die hinreichenden Bedingungen in XXIII., \$ 6. angegeben sind, geben diese sämmtlichen Combinationen solche $S_{p-\mu}$ gerade Charakteristiken $(a)$ und $R_{p-\mu}$ ungerade Charakteristiken (b), und zwar jede nur einmal, welche in einem System von $\mu$ Gruppenpaaren der Art XVI.:

wo

$$
\left[r_{1}\right],\left[s_{1}\right] ;\left[r_{2}\right],\left[s_{2}\right] ; \cdots ;\left[r_{\mu}\right],\left[s_{\mu}\right],
$$

$$
K_{r_{i}, s_{i}} \equiv 1 ; \quad K_{r_{i}, r_{k}} \equiv K_{r_{i}, s_{k}} \equiv K_{s_{i}, s_{k}} \equiv 0 ; \quad\left[r_{i} s_{i}\right]=\left[t_{i}\right] \text {, }
$$

enthalten sind. Daher erhalten sämmtliche $2^{2 p}$ Charakteristiken und $2^{2 p}-1$ Gruppen die Darstellung XX., § 4. Man stellt ein solches System von $2(p-\mu)+1$ Charakteristiken her, indem man von einem System von $\mu+1$ Gruppenpaaren der Art XVI.:

$$
\left[r_{1}\right],\left[\mu_{1}\right] ; \cdots ;\left[r_{\mu}\right],\left[s_{\mu}\right] ;\left[r_{\mu+1}\right],\left[s_{\mu+1}\right]
$$

ausgeht, aus den darin enthaltenen Charakteristiken ein der obigen Definition entsprechendes System von $2(p-\mu)-1$ Charakteristiken

$$
(c) \equiv\left(c_{1}\right),\left(c_{2}\right), \cdots,\left(c_{2(p-\mu)-1}\right)
$$

herausnimmt und hieraus das System bildet: 
1) wenn $p-\mu$ ungerade:

$$
\left(c t_{\mu+1}\right) \equiv\left(c_{1}\right),\left(c_{2}\right), \cdots,\left(c_{2(p-\mu)-1}\right),\left(c r_{\mu+1}\right),\left(c s_{\mu+1}\right) ;
$$

2) wenn $p-\mu$ gerade:

$$
(c) \equiv\left(c_{1} t_{\mu+1}\right),\left(c_{2} t_{\mu+1}\right), \cdots,\left(c_{3(p-\mu)-1} t_{\mu+1}\right),\left(c r_{\mu+1}\right),\left(c s_{\mu+1}\right) \text {. }
$$

Auf diesem Wege ergeben sich auch alle Systeme. In dem Falle 2), wenn $p-\mu$ gerade ist, kann man auch die $2(p-\mu+1)$ Charakteristiken:

$$
\left.(c),\left(c_{1} t_{\mu+1}\right),\left(c_{2} t_{\mu+1}\right), \cdots,\left(c_{2(p-\mu)-1} t_{\mu+1}\right),\left(c r_{\mu+1}\right),\left(c s_{\mu+1}\right) \equiv 0\right)
$$

zu einem System von der Summe 0 zusammenfassen.

\section{$\S 8$.}

Die ,ausgezeichneten“ Systeme von $2 p+1$ Charakteristiken.

1. Unter den im vorhergehenden Paragraphen entwickelten Systemen von $2(p-\mu)+1$ Charakteristiken sind diejenigen für $\mu=0$, also von $2 p+1$ Charakteristiken, ausgezeichnet, insofern nämlich die Combinationen der $2 p+1$ Charakteristiken eines solchen Systems alle überhaupt existirenden Charakteristiken und Gruppen, und jede nur einmal, liefern. Solche $2 p+1$ Charakteristiken eignen sich also, da weiter aus der Zusammensetzung ihrer Combinationen unmittelbar deren Eigenschaft als Gruppen - oder als eigentliche, und zwar als gerade oder als ungerade eigentliche Charakteristik zu erkennen ist, am besten zur Darstellung aller Beziehungen 2 wischen den Charakteristiken; und ihre Systeme mögen daher als "ausgezeichnete" werden. - Es ist dabei zu betonen, dass sämmtliche $2^{2 p}-1$ Gruppencharakteristiken sich schon aus $2 p$ solchen darstellen, nämlich aus den Combinationen von $2 p+1$ Gruppen $\left[c c_{t}\right]$, deren Summe $=0$ ist, wenn das anggezeichnete System mit

$$
(c)=\left(c_{1}\right),\left(c_{2}\right), \cdots,\left(c_{2 p+1}\right)
$$

bezeichnet wird; dass aber, was ebenso wesentlich ist, die eigentlichen Charakteristiken aus nicht weniger als $2 p+1$ solchen dargestellt werden können.

XXVI. Definirt man ein System von (für $p=4 n$ oder $4 n+1$ ) geraden [(für $p=4 n+2$ oder $4 n+3)$ ungeraden] Charalteristiken:

$$
\left(c_{1}\right),\left(c_{2}\right), \cdots,\left(c_{2 p+1}\right)
$$

durch die Bedingung, dass eine Charakteristik (c) existire, fïr welche alle

$$
\left(e c_{t} e_{x}\right)
$$

von entgegengesetztem Charahter (des Geraden oder Ungeraden) sind, wie (c), so hat man dieses System als ausgezeichnetes und zugleich als, vollständiges" zu bezeichnen; nämlich von dex Art, dass keine $2(p+1)^{10}$ 
gerade [ungerade] Charahteristik $c_{2(p+1)}$ hinzugefunden werden kann, für welche alle

$$
\left(c c_{\iota} c_{2(p+1)}\right)
$$

von entgegengesetztem Charakter wie (c) sind. Zugleich wird (c) gleich der Summe der Charakteristiken.

Denn nach XXIII., § 6., folgt aus dieser Definition, dass alle

$$
\left(c_{2} c_{\mathrm{x}} c_{2}\right)
$$

ungerade [gerade] sind; dass also das System eimes unserer „ausgezeichneten " ist. Hiernach lassen sich alle Charakteristiken aus den $2 p+1$ Grössen des Systems zusammensetzen, woraus sogleich hervorgeht, dass heine andere Zusanmensetzung, als die Summe der $2 p+1$ Charakteristiken, die ron $(c)$ geforderte Eigenschaft hat. Eine weitere $2(p+1)^{\text {te }}$ Charakteristik kanu dann nach dieser Definitiou nicht zu dem System von $2 p+1$ Grössen binzutreten. Denn diese wäre entweder die Summe $(c)$ selbst: daun aber müssten $(c)$ und $\left(c_{t}\right)$ von gleichem Cbarakter, aber auch, wegen $(c)$ und $\left(c c c_{t}\right)=\left(c_{t}\right)$, von entgegengesetztem Charakter sein; oder die hinzutretende Charakteristik wäre vou (c) verschieden: damn würden die Combinationen der $2(p+1)$ Charakteristiken, genau wie in 2 . $\$ 6$. folgt, alle von einander verschieden sein und mehr als $S_{p}$ gerade und $R_{p}$ ungerade Charakteristiken liefern, was nicht existirt.

XXVI'. Definirt man dagegen das ausgezeichnete System

$$
\left(c_{1}\right),\left(c_{2}\right), \cdots,\left(c_{2 p+1}\right)
$$

durch die Bedingungen, dass alle

$$
\begin{array}{r}
\left(c_{1}\right) \text { gerade [ungerade], } \\
\left(c_{4} c_{x} c_{2}\right) \text { ungerade [gerade] }
\end{array}
$$

sein sollen, so kann dasselle nur für ungerade $p$ als "vollständig" Zezeichnet werden. Für gerade $p$ dagegen tritt noch eine $2(p+1)^{\text {te }}$ Charakteristik hinzu, welche den gegebenen Bedingungen genügt: die Summe (c) der $2 p+1$ Charakteristiken; so dass für gerade $p$ dic "vollständigen" Systeme aus $2 p+2$ Charakteristiken von der Summe 0 bestehen:

wo die

$$
0=(c),\left(c_{1}\right),\left(c_{2}\right), \therefore\left(c_{2 p+1}\right),
$$

$$
\begin{gathered}
\left(c_{\varepsilon}\right) \text { tür } p=4 n \text { gerade, für } p=4 n+2 \text { ungerade, } \\
\left(c_{4} c_{x} c_{2}\right) ", \text { ungerade, " } ", \text { gerade }
\end{gathered}
$$

sind, wenn $\iota, x, \lambda$ alle von einander verschiedenen Werthe

annehmen.

$$
0,1, \cdots, 2 p+1
$$

Denn $(c)$ selbst ist nur für gerade $p$ mit den $c_{t}$ zugleich gerade oder ungerade; eine von $(e)$ versebiedene Charakieristik kann aber, wie beim Beweise von XXVI. bemerbt ist, nicht hinzutreteu. 
2. Wir geben noch ein Beispiel zu diesen ausgezeichneten Systemen, inden wir das Beispiel $\S 5$., 1. mit der dortigen Bezeichnung weiter entwickeln.

In den $2 p$ Gruppenpaaren:

$$
\left[\varrho_{p}\right],\left[\sigma_{p}\right] ;\left[\varrho_{p-1}\right],\left[\sigma_{p-1}\right] ; \cdots ;\left[\rho_{1}\right],\left[\sigma_{1}\right]
$$

ist allein die gerade Charakteristik (0) enthalten. Hieraus ergeben sich nach $\$ 7$. der Reihe nach folgende Systeme von 3, 5, 7, . . 2p+1 Charakteristiken :

$$
\begin{aligned}
&\left(\tau_{1}\right) \equiv(0),\left(\varrho_{1}\right),\left(\sigma_{1}\right) ; \\
&\left(\tau_{1}\right) \equiv\left(\tau_{2}\right),\left(\varrho_{1} \tau_{2}\right),\left(\sigma_{1} \tau_{3}\right),\left(\tau_{1} \varrho_{2}\right),\left(\tau_{1} \sigma_{2}\right) ; \\
&\left(\tau_{1} \tau_{3}\right) \equiv\left(\tau_{2}\right),\left(\varrho_{1} \tau_{2}\right),\left(\sigma_{1} \tau_{2}\right),\left(\tau_{1} \varrho_{1}\right),\left(\tau_{1} \sigma_{2}\right),\left(\tau_{1} \varrho_{3}\right),\left(\tau_{1} \sigma_{3}\right) ; \\
&\left(\tau_{1} \tau_{3}\right) \equiv\left(\tau_{2} \tau_{4}\right),\left(\varrho_{1} \tau_{2} \tau_{4}\right),\left(\sigma_{1} \tau_{2} \tau_{4}\right),\left(\tau_{1} \varrho_{2} \tau_{3}\right),\left(\tau_{1} \sigma_{2} \tau_{4}\right),\left(\tau_{1} o_{3} \tau_{4}\right), \\
& \quad\left(\tau_{1} \sigma_{3} \tau_{4}\right),\left(\tau_{1} \tau_{2} \varrho_{4}\right),\left(\tau_{1} \tau_{3} \sigma_{4}\right) ; \\
&\left(\tau_{1} \tau_{3} \tau_{5}\right) \equiv\left(\tau_{2} \tau_{4}\right),\left(\varrho_{1} \tau_{2} \tau_{4}\right),\left(\sigma_{1} \tau_{2} \tau_{4}\right),\left(\tau_{1} \varrho_{2} \tau_{4}\right),\left(\tau_{1} \sigma_{2} \tau_{4}\right),\left(\tau_{1} \varrho_{3} \tau_{4}\right), \\
& \text { ete. } \quad\left(\tau_{1} \sigma_{3} \tau_{4}\right),\left(\tau_{1} \tau_{3} \varrho_{4}\right),\left(\tau_{1} \tau_{3} \sigma_{4}\right),\left(\tau_{1} \tau_{3} \varrho_{5}\right),\left(\tau_{1} \tau_{3} \sigma_{3}\right) ;
\end{aligned}
$$

Das Bildungsgesetz dieser Systeme wird klarer, wenn man die Summe eines solchen zu seinen sämmtlichen Charakteristiken hinzuaddirt. So erbält man aus dem System von 11 Charakteristiken mit Summe $\left(\tau_{1} \tau_{3} \tau_{5}\right)$ :

$$
\begin{aligned}
0 \equiv\left[\tau_{1} \tau_{2} \tau_{3} \tau_{4} \tau_{5}\right], & {\left[\varrho_{1} \tau_{2} \tau_{3} \tau_{4} \tau_{5}\right],\left[\varrho_{2} \tau_{3} \tau_{4} \tau_{5}\right],\left[\varrho_{3} \tau_{4} \tau_{5}\right],\left[\varrho_{4} \tau_{5}\right],\left[\varrho_{5}\right], } \\
& {\left[\sigma_{1} \tau_{2} \tau_{3} \tau_{4} \tau_{5}\right],\left[\sigma_{2} \tau_{3} \tau_{4} \tau_{3}\right],\left[\sigma_{3} \tau_{4} \tau_{5}\right],\left[\sigma_{4} \tau_{5}\right],\left[\sigma_{5}\right], }
\end{aligned}
$$

Gruppen, welche alle zu zwei die Beziehung $K \equiv 1$ zu einander haben. Man bilde ebenso allgemein die $2 p+1$ Gruppen von der Beziehung $K \equiv$ : :

$$
\begin{array}{r}
{\left[\tau_{1} \tau_{1,} \tau_{3} \cdots \tau_{p}\right],\left[\varrho_{1} \tau_{2} \tau_{3} \cdots \tau_{p}\right],\left[\varrho_{2} \tau_{3} \tau_{4} \cdots \tau_{p}\right], \cdots,\left[\varrho_{p-1} \tau_{p}\right],\left[\varrho_{p}\right],} \\
{\left[\sigma_{1} \tau_{2} \tau_{3} \cdots \tau_{p}\right],\left[\sigma_{2} \tau_{3} \tau_{4} \cdots \tau_{p}\right], \cdots,\left[\sigma_{p-1} \tau_{p}\right],\left[\sigma_{p}\right],}
\end{array}
$$

und addire zu jeder Gruppe die Charakteristik

$$
\begin{aligned}
& \left(\tau_{1} \tau_{3} \tau_{5} \cdot \tau_{p-1}\right), \text { wenn } p \text { gerade, } \\
& \left(\tau_{1} \tau_{3} \tau_{3} \cdots \tau_{p}\right), \quad " \Rightarrow \text { ungerade, }
\end{aligned}
$$

so erhält man ein ausgezeichnetes System von $2 p+1$ Charakteristiken mit Summe $\left(\tau_{1} \tau_{3} \cdots \tau_{p-1}\right)$, bez. $\left(\tau_{1} \tau_{3} \cdots \tau_{p}\right)$.

Um hieraus sämmtliche existirende ausgeceichnete Systeme zu erhalten, kann man auf das vorliegende System die in $\$ 5$. 3. entwickelten Substitutiouen ausführen, und eriālt dann, wenn $(g)$ die in einem System voll $p$ Gruppenpaaren der Art XVI.

$$
\left[r_{1}\right],\left[s_{1}\right] ; \cdots ;\left[r_{p}\right],\left[s_{p}\right] \text {, }
$$

*) Solcher Art ist das ron Weierstrass, Küuigsberger, Prym und schottky, an den citirten Orteu, henutate System. 
enthaltene eine gerade Charakteristik vorstellt, das allgemeinste System in der f'orm:

$$
\begin{array}{r}
{\left[t_{p} t_{p-1} t_{p-2} \cdots t_{1}\right],\left[r_{p} t_{p-1} t_{p-2} \cdots t_{1}\right],\left[r_{p-1} t_{p-2} t_{p-3} \cdots t_{1}\right], \cdots,\left[r_{2} t_{1}\right],\left[r_{1}\right],} \\
{\left[s_{p} t_{p-1} t_{p-2} \cdots t_{1}\right],\left[s_{p-1} t_{p-2} t_{p-3} \cdots t_{1}\right], \cdots,\left[s_{2} t_{1}\right],\left[s_{1}\right],}
\end{array}
$$

wo noch zu jeder dieser $2 p+1$ Gruppen die Charakteristik

$\mathrm{zu}$ addiren ist.

$$
\begin{aligned}
& \left(g t_{2} t_{1} \cdots t_{p}\right), \text { wenn } p \text { gerade, } \\
& \left(g t_{1} t_{3} \cdots t_{p}\right) \text {, wenn } p \text { ungerade, }
\end{aligned}
$$

$\$ 9$.

Weitere Charakteristikensysteme.

A usser den in $\$ 7$. gegebenen Systemen von $2 \pi+1$ Charakteristiken existiren noch weitere: 1) solche von ebensovielen Charakteristiken, aber von entgegengesetztem Charakter des Geraden oder Ungeraden, wie die dortigen; für $\pi=0,1, \ldots, p-1$; 2) solche, die aus einer geraden Anzabl von Charakteristiken bestehen.

1) Sei

$$
(c) \equiv\left(c_{1}\right),\left(c_{2}\right), \cdots,\left(c_{2(p-\mu)+1}\right)
$$

eines unserer früheren Systeme von $2(p-\mu)+1$ Charalteristiken, gebildet aus den in den $2 \mu$ Gruppen

$$
\left[r_{1}\right],\left[s_{1}\right] ; \cdots ;\left[r_{\mu-1}\right],\left[s_{\mu-1}\right] ;\left[r_{\mu}\right],\left[s_{\mu}\right]
$$

enthaltenen Charakteristiken. Man hat dann in

$$
\left(c t_{\mu}\right) \equiv\left(c_{1} t_{\mu}\right),\left(c_{2} t_{\mu}\right), \cdots,\left(c_{2(p-\mu)+1} t_{\mu}\right)
$$

ein System von $2(p-\mu)+1$ Charakteristiken der gesuehten Art, wo $\mu$ die Werthe $p-1, \cdots, 2,1$ annehmen kann.

Dass auf diese Weise alle solche Systeme erhalten werden, ergiebt sich durch den Uebergang von $\mu+1$ auf $\mu$, genan wie in $\S 7$., 2. und 3 .

Im Falle hier $p-\mu$ gerade ist, ist die Summe $\left(c t_{\mu}\right)$ mit den $\left(c_{1} t_{\mu}\right)$ zugleich gerade oder zugleich ungerade; man hat also in

$$
0 \equiv\left(c t_{\mu}\right),\left(c_{1} t_{\mu}\right),\left(c_{2} t_{\mu}\right), \cdots,\left(c_{2(p-\mu)+1} t_{\mu}\right)
$$

ein System von $2(p-\mu+1)$ Charakteristiken, die ron entgegengesetztem Charakter sind, wie die der entsprechenden Systeme des $\S 7 ., 4 .$, und die Summe 0 geben.

2) Ausser den schon in $\$ 7 ., 4$. und in diesem \$1) enthaltenen Systemen, die aus einer geraden Auzahl von Charakteristiken bestehen - nämlich solchen, die aus $4 n+2$ Charaliteristiken bestehen, deren Summe $=0$ ist - , lassen sich noch weitere bilden.

Seien die

$$
(c) \equiv\left(c_{1}\right),\left(c_{2}\right), \cdots,\left(c_{2(p-\mu)+1}\right),
$$


und die Gruppen

$$
\left[r_{1}\right],\left[s_{1}\right] ; \cdots ;\left[r_{\mu}\right],\left[s_{\mu}\right]
$$

wie oben in 1) definirt.

Ist nun erstens $p-\mu$ ungerade, so hat man in

$$
\left[t_{\mu}\right] \equiv\left(c_{1}\right),\left(c_{2}\right), \cdots,\left(c_{2(p-\mu)+1}\right),\left(c t_{\mu}\right)
$$

ein System von $2(p-\mu+1)$ Charakteristiken (gerade für $p-\mu=4 n+1$, ungerade für $p-\mu=4 n+3)$, die alle in den Gruppen

$$
\left.\left[r_{1}\right],\left[s_{1}\right] ; \cdots ; \mid r_{\mu-1}\right],\left[s_{\mu-1}\right]
$$

enthalten sind, deren Summe richt verschwindet, und die, zu 3 combinirt, Summen von entgegengesetztem Charakter liefern, als die $\left(c_{\imath}\right)$. Dies gilt für $\mu=p-1, p-3, \cdots, 2$ bez. 1 . Ferner hat man in

$$
\left[t_{\mu}\right] \equiv\left(c_{1} t_{\mu}\right),\left(c_{2} t_{\mu}\right), \cdots,\left(c_{2(p-\mu)+1} t_{\mu}\right),(c)
$$

ein analoges System von $2(p-\mu+1)$ Charakteristiken, die für $p-\mu=4 n+1$ ungerade, für $p-\mu=4 n+3$ gerade sind.

Ist zweitens $p-\mu$ gerade, so erhält man durch analoges Verfahren nur wieder die schon oben betrachteten Systeme mit Summe 0, oder die folgenden :

$$
\begin{aligned}
{\left[r_{\mu}\right] } & \equiv\left(c_{1}\right),\left(c_{2}\right), \cdots,\left(c_{2(p-\mu)+1}\right),\left(c r_{\mu}\right), \quad \text { wo } \mu \geqq 1, \\
{\left[t_{\mu-1} t_{\mu}\right] } & \equiv\left(c_{1} t_{\mu},\left(c_{2} t_{\mu}\right) \cdots,\left(c_{2(p-\mu)+1} t_{\mu}\right),\left(c t_{\mu-1}\right), \text { wo } \mu \geq 2 .\right.
\end{aligned}
$$

Ist insbesondere $p$ gerade, und setzt man $\mu=1$, so ergiebt sich aus dem ersten Falle ein System

$$
[d] \equiv\left(d_{1}\right),\left(d_{2}\right), \cdots,\left(d_{2 p}\right)
$$

von $2 p$ Charakteristiken, die für $p=4 n$ ungerade, für $p=4 n+2$ gerade sind. Dies System ist zuglesch ein vollständiges, insofern keine weitere ungerade [gerade] Charakteristik $\left(d_{2 p+1}\right)$ hinzugefunden werden kann, für welche alle

$$
\left(d_{t} d_{x} d_{2 p+1}\right)
$$

gerade [ungerade] sind; denn andernfalls würden die Combinationen des Systems nach XXIV. mehr Charakteristiken liefern, als überhaupt existiren.

Fasst man alles zusammen, so ergiebt sich, dass überhaupt folgende Systeme existiren, in denen immer die Summen je dreier Charakteristiken von entgegengesetztem Charakter sind, als die Charakteristiken selbst:

XXVII. Es giebt, für jedes $p$, Systeme von $2 \pi+1$ geraden und Systeme von $2 \pi+1$ ungeraden Charakteristiken, für $\pi=0,1, \cdots, p-1$; und ebensolche Systeme von $2 \pi$ Charalteristitien, deren Summe nicht $=0$ ist. Ferner existiren für jedes $p$ Systeme von $4 \pi+2$ geraden und von $4 \pi+2$ ungeraden Charaliteristiken, deren Summe $=0$ ist, für $\pi=1,2 \ldots \frac{p-2}{2}$, bez. $\frac{p-1}{2}$. Dazu kommen noch: 
1) für $p=4 n[4 n+2]$ die ausgezeichneten Systeme von $2 p+1$ geraden [ungeraden] Charakteristiken, die vollständigen Systeme von $2 p+2$ geraden [ungeraden] Charakteristiken, deren Summe $=0$ ist, und die vollständigen Systence von $2 p$ ungeraden [geraden] Charahteristiken, deren Summe nicht $=0$ ist;

2) für $p=4 n+1[4 n+3]$ : die ausgezeichneten und zugleich vollständigen Systeme von $2 p+1$ geraden [ungeraden] Charakteristiken.

\section{$\S 10$.}

\section{Anzahl der Charakteristikensysteme.}

Wir bestimmen bier die Anzahl der verschiedenen, in den vorhergehenden Paragraphen gegebenen Charakteristikensysteme. Hierzu führt die dort ausgeführte Bildungsweise dieser Systeme.

1. Die Anzahl der in $\$ 7$. behandelten Systeme von $2(p-\mu)+1$ Charakteristiken, die für $p-\mu=4 n, 4 n+1$ gerade, für $p-\mu=4 n+2$, $4 n+3$ ungerade sind, sei $L_{\mu}$. Sei ein solches System von $2(p-\mu)-1$ Charakteristiken:

$$
(c) \equiv\left(c_{1}\right),\left(c_{2}\right), \cdots,\left(c_{2(p-\mu)-1}\right),
$$

enthalten in den $\mu+1$ Gruppenpaaren:

$$
\left[r_{1}\right],\left[s_{1}\right] ; \cdots ;\left[r_{\mu}\right],\left[s_{\mu}\right] ;\left[r_{\mu+1}\right],\left[s_{\mu+1}\right] .
$$

Diese $2(p-\mu)-1$ Charakteristiken sind dann zugleich, wie beim Beweise der Zahl $N_{\mu}$ des Salzes XXI., $\S 5$. gezeigt ist, in

$$
\left(S_{\mu+1}-1\right) 2^{2 \mu-1}=R_{\mu+1} S_{\mu}
$$

Gruppenpaaren dieser Art enthalten, und jedes solches Paar liefert nach $\$ 7$., 1. ein System

$$
(d) \equiv\left(d_{1}\right),\left(d_{2}\right), \cdots,\left(d_{2(p-\mu)+1}\right)
$$

von $2(p--\mu)+1$ Charakteristiken. Umgekehrt wird jedes System $(d)$ auf diese Art, nach $\$ 7 ., 2$. erhalten, und zwar auf

$$
\frac{[2(p-\mu)+1] 2(p-\mu)}{1 \cdot 2}
$$

Arten, da man dabei irgend zwei der $\left(d_{t}\right)$ auszeichnen kann, Indem man also von allen $L_{k+1}$ Systemen von $2(p-\mu)-1$ Charakteristiken ausgeht, findet man

$$
L_{\mu}=\frac{\left(S_{\mu+1}-1\right) 2^{2 \mu}}{\left[{ }^{2}(p-\mu)+1\right]^{2}(p-\mu)} \cdot L_{\mu+1}=-\frac{2 R_{\mu+1} S_{\mu}}{[2(p-\mu)+1]^{2}(p-\mu)} L_{\mu+1} .
$$
daher:

A ber $L_{p}$ ist gleich der Anzahl der geraden Charakteristiken, $=S_{p}$,

$$
\begin{gathered}
L_{\mu}=\frac{\left(S_{\mu+1}-1\right)\left(S_{\mu+2}-1\right) \cdots\left(S_{p}-1\right) \cdot S_{p} \cdot c_{2}(p+\mu-1(p-\mu)}{1 \cdot 2 \cdot 3 \cdot 4 \cdots(2 p-2 \mu+1)} \\
=\frac{S_{\mu} \cdot R_{2(\mu+1)} R_{2(\mu+2)} \cdots R_{2 p}}{1 \cdot 2 \cdot 3 \cdots(2 p-2 \mu+1)} .
\end{gathered}
$$


Hieraus, und aus den Zahlen $M_{\mu}, N_{\mu}$ des Satzes XXI., § 5., folgt weiter, dass die Anzahl derjenigen dieser Systeme, welche aus $2(p-\mu)+1$ Charakteristiken gebildet sind, die in gegebenen $\mu$ Gruppenpaaren

$$
\left[r_{1}\right],\left[s_{1}\right] ; \cdots ;\left[r_{u}\right],\left[s_{\mu}\right]
$$

enthalten sind, beträgt:

$$
L_{\mu} \cdot \frac{N_{\mu}}{M_{\mu}^{-}}=\frac{R_{2(p-\mu)} \cdot R_{2(p-\mu-1)} \cdots R_{1} \cdot R_{2}}{1 \cdot 2 \cdot 3 \cdots \cdot(2 p-2 \mu+1)}
$$

Insbesondere wird die Anzahl $L_{i}$ der ausgezeichneten Systeme von $2 p+1$ Charakteristiken:

$$
L_{0}=\frac{R_{2} \cdot R_{1} \cdots R_{2 p}}{1 \cdot 2 \cdot 3 \cdots(2 p+1)} .
$$

2. Die Anzahl $L_{\mu}{ }^{\prime}$ der Systeme von $2(p-\mu)+1$ Charakteristiken, die für $p-\mu=4 n, 4 n+1$ ungerade, für $p-\mu=4 n+2,4 n+3$ gerade sind, ergiebt sich aus $L_{u}$, nach $\S 9 ., 1$.

Hiernach führt jedes System der vorigen Art 1.

$$
(c) \equiv\left(c_{1}\right),\left(c_{2}\right), \cdots,\left(c_{2(\nu-\mu)+1}\right)
$$

zu einem System dieser Art 2., indem man irgend eine der $R_{\mu}$ Gruppencharakteristiken $[v]$, welche, nach $\S 4$., XIX. und XX., die $c_{\text {s }}$ und ihre Combinationen $\mathrm{zu} 3,5, \ldots$ nicht enthalten, zu den $c$, addirt. Umgekehrt ergiebt die Darstellung der Gruppen in XX., dass jedes solche System 2., wie

$$
\left(c_{1} t_{1}\right),\left(c_{2} t_{1}\right), \cdots,\left(c_{2(j-\beta)+1} t_{1}\right)
$$

durch Addition irgend einer der $S_{\mu}$ Gruppencharakteristiken, welche die $\left(c_{t} t_{1}\right)$ nicht enthalten, auf ein System der Art 1. zurückgeführt werden kann. Daher folgt:

$$
L_{\mu}^{\prime}=\frac{R_{\mu}}{S_{\mu}} \cdot L_{\mu}=\frac{R_{\mu} \cdot R_{2(\mu+1)} \cdot R_{2(\mu+2)} \cdots R_{2 p}}{1 \cdot 2 \cdot 3 \cdots(2 p-2 \mu+1)} .
$$

3. Die Anzahl der Systeme von $4 \pi+2$ Charakteristiken, deren Summe $=0$ ist, ergiebt sich aus der unter 1 ., bez. unter 2 ., bestimmten Anzahl $L_{p-2 \pi}$, bez. $L_{p-2 \pi}^{s}$, der Systeme von $4 \pi+1$ Charakteristiken, indem man diese Zabl nur durch $4 \pi+2$ dividirt. Denn man erbält aus jedem dieser Systeme eines von $4 \pi+2$, durch ' Zufügung der Summe der $4 \pi+1$ Charakteristiken; aus jedew System von $4 \pi+2$ aber $4 \pi+2$ Systeme von je $4 \pi+1$, durch Weglassung irgend einer der $4 \pi+2$ Charakteristiken.

4. Die Anzahl der Systeme von $2(p-\mu+1)$ Charakteristiken, deren Summe nicht $=0$ ist, folgt aus $\$ 9 ., 2$.

Ist erstens $p-\mu$ ungerade, so ergeben sich dort aus jedem der $L_{\mu}$ Systeme 1. von $2(p-\mu)+1$ Charakteristiken $R_{\mu}$ Systeme von $2(p-\mu+1)$; und aus jedem dieser, durch Weglassung irgend einer 
seiner Charakteristiken, $2(p-\mu+1)$ solcher ron $2(p-\mu)+1$. Man erhält also

$$
\frac{L_{\mu} \cdot R_{\mu}}{2(p-\mu+1)}=\frac{1}{2} \frac{R_{2 \mu} R_{2(\mu+1)} \cdots R_{2 p}}{1 \cdot 2 \cdot 3 \cdots(2 p-2 \mu+2)}
$$

Systeme von $2(p-\mu+1)$ Charaliteristiken vou demselben Charakter wie 1. Ebenso ergeben sich aus 2.

$$
\frac{L_{\mu}^{\prime} \cdot S_{\mu}}{2(p-\mu+1)}=\frac{L_{\mu} \cdot R_{\mu}}{2(p-\mu+1)}
$$

Systeme von $2(p-\mu+1)$ Charakteristiken von entgegengesetztem Charakter wie 1.; insbesondere, für $\mu=1$ und gerade $p$, als Anzahl der vollständigen Systeme von $2 p$ Charakteristiken:

$$
\frac{L_{1}}{2 p}=\frac{2 p+1}{2} \cdot L_{0}
$$

Ist zweitens $p-\mu$ gerade, so folgen analog:

$$
\frac{L_{\mu}\left(S_{\mu}-1\right)}{2(p-\mu+1)}
$$

Systeme von $2(p-\mu+1)$ Charakteristiken vom Charakter wie 1., und

$$
L_{u} \cdot \frac{R_{\mu} \cdot\left(2^{2 \mu-2}-1\right)}{S_{\mu}(2 p-2 \mu+2)}=\frac{L_{\mu}^{\prime} \cdot\left(2^{2 \mu-2}-1\right)}{2(p-\mu+1)}
$$

Systeme von $2(p-\mu+1)$ Charahteristiken von entgegengesetztem Charakter wie 1.

5. Aus dem Vorhergehenden folgt allgemein, wie oft man ein System von $\boldsymbol{M}$ Charakteristiken zu einem System von $\boldsymbol{N}(\boldsymbol{N}>\boldsymbol{M})$ Charakteristiken, welche mit den ersteren zugleich gerade oder zugleich ungerade sind, ergänzen kann. Wenn es nämlich von den ersteren Systemen überhaupt $Q_{M}$, von den letzteren Systemen $Q_{N}$ giebt, wo die Zahlen $Q_{M}$ und $Q_{N}$ nach $1 ., \cdots, 4$. dieses Paragraphen bestimmt werden müssen, so kommt, da jedes System von $N$ Charakteristiken durch Weglassung von irgend $N-M$ derselben auf ein System von $M$ führt, jedes solches System von $M$ Charakteristiken in

Systemen von $N$ Charakteristiken vor.

$$
\frac{N(N-1)(N-2) \cdots(M+1)}{1 \cdot 2 \cdot 3 \cdots(N-M)} \cdot \frac{Q_{N}}{Q_{M I}}
$$

\section{$\S 11$.}

\section{Zweitheilungsgleichungen.}

Für diejenigen Gleichungen, deren Wurzeln man eindeutig den $R_{p}$ ungeraden oder den $S_{p}$ geraden eigentlichen Charakteristiken, oder den $2^{2 p}-1$ Gruppencharakteristiken, etc., zuordnen kann, 
haben wir in $\S 5$. 3. die ihnen zugehörige Substitutionsgruppe von der Ordnung

entwickelt.

$$
Z=R_{2 p} \cdot R_{2(p-1)} \cdots R_{i} R_{i}
$$

Zunächst ist dabei zu bemerken, dass diese Substitutionen für $p>2$ alle aus einer geraden Zahl von Transpositionen der eigentlichen Charakteristiken bestelen. Nur für $p=2$ und $p=1$ werden die Substitutionen aus solchen zusammengesetzt, welche bezügl. aus $S_{1}=3$ und $S_{0}=1$ Transpositionen der geraden Charakteristiken bestehen; und in diesen beiden Fällen kann man daher, indem man zuerst die Quadratwurzel aus der Discriminante der betreffenden Gleichung nimmt, die Gruppe sogleich auf die Ordnung $\frac{R_{4} R_{2}}{2}=3 \cdot 120$, bez. $\frac{R_{z}}{2}=3$, erniedrigen. Für $p=2$ hat man eine allgemeine Gleichung $6^{\text {ten }}$, für $p=1$ eine solche $3^{\text {ten }}$ Grades. Sei weiterhin $p>2$.

Die in $\S \S 4,7 ., 8 ., 9$. entwickelten Systeme bieten sämmtlich Mittel, um Resolventen der vorgegebenen Gleichungen zu definiren. Aus der Bildungsweise der Systeme von $\mu$ Gruppenparen des $\S 4$ :

$$
\left[r_{1}\right],\left[s_{1}\right] ; \cdots ;\left[r_{\mu}\right],\left[s_{\mu}\right]
$$

geht hervor, dass unsere Substitutionen erlauben, alle solche Systeme in einander überzuführen und ferner die verschiedenen Paare eines Systems mit einander, sowie die Gruppen eines jeden Paares unter sich zu vertauschen. Hieraus folgt, nach $\S 7$. ff., weiter, dass vermöge unserer Substitutionen auch jedes der Systeme ron $N$ Charakteristiken der $\$ \S 7 .-9$, in jedes andere derselben Art übergeführt werden kann, nämlich in ein solches von $N$ Charakteristiken, die mit den ersteren zugleich gerade oder ungerade sind, und deren Summe mit der des ersteren Systems zugleich verschwindet oder nicht; und dass weiter auch noch die Charakteristiken eines Systems beliebig mit einander vertauscht werden können.

Kennt man nun diejenigen $2 p$ Functionen der Wurzeln der gegebenen Gleichung, welche man den $2 p$ Gruppen eines Systems des $\$ 4$ :

$$
\left[r_{1}\right],\left[s_{1}\right] ;\left[r_{2}\right],\left[s_{2}\right] ; \cdots ;\left[r_{p}\right],\left[s_{p}\right]
$$

zuordnen kann, so ergeben sich bieraus alle Charakteristiken und Gruppen, also alle Wurzeln der Gleichung, eindeutig. Kennt man ebenso diejenigen $2 p+1$ Wurzeln oder Functionen der Wurzelu der gegebenen Gleichung, welche man den $2 p+1$ Charakteristiken eines ausgezeichneten Systems zuordnen kamn, so ergeben sich wiederum durch deren Combinationen alle Charakteristiken und Gruppen, d. h. alle Wurzeln, eindeutig. Hiernach kann man zur Auflösung der gegebenen Gleichung so verfahren: 
1. Man bestimmt durch Aufsuebung einer Wurzel einer Gleichung vom Grade $R_{2 p}$ zuerst die beiden Gruppen eines Paares, $\left[r_{1}\right],\left[s_{1}\right]$; wonach die Substitutionsgruppe anf eine solche von der Ordnung

$$
Z_{1}=R_{2(p-1)} R_{2(p-2)} \cdots R_{2}
$$

zurückkommt, die isomorph ist mit derjenigen, die zu den $(p-1)$ reihigen Charakteristiken gehört. Aehnlich bestimmt man dann weiter durch Aufsuchung je einer Wurzel von Gleichungen der Grade $R_{2(p-1)}$, $R_{2(y-2)}, \cdots, R_{2}$ der Reihe nach die übrigen Gruppen $\left[r_{2}\right],\left[s_{2}\right] ; \cdots$; $\left[r_{p}\right],\left[s_{p}\right]$.

Dieses Verfahren kann mannigfach modificirt werden:

a) Um ein Paar $\left[r_{1}\right],\left[s_{1}\right]$ zu bestimmen, kaun man zunächst vou derjenigen Gleichung vom Grade $2^{2 p}-1$, welche die sämmtlichen Gruppen bestimmt, eine Wurzel $\left[r_{1}\right]$ aufsuchen; sodann ron der Gleichung des Grades $2^{2 p-1}$, welche die Gruppen $\left[s_{1}\right]$ liefert, die mit $\left[r_{1}\right]$ die Beziehung $K_{r_{1}, s_{1}}=1$ haben, eine Wurzel. Ebenso in Bezug auf die übrigen Paare $\left[r_{2}\right],\left[s_{2}\right]$; etc., so dass man also im Ganzen der Reibe nach je eine Wurzel von Gleichungen der Grade

$$
2^{2 \rho}-1,2^{2 p-1}, 2^{2(p-1)}-1,2^{2(p-1)-1}, \cdots, 2^{2}-1,2^{1}
$$

zu suchen hat. - Dabei ist ferner zu beachten, dass man in dieser Art nur bis zur Aufsuchung von $\left[r_{p-2}\right],\left[s_{p-2}\right]$ zu gehen braucht; zur Aufsuchung von $\left[r_{p-1}\right],\left[s_{p-1}\right] ;\left[r_{p}\right],\left[s_{p}\right]$ aber die oben bei $p=2$ und $p=1$ augegebenen Vereinfachungen, durch vorheriges Ausziehen von Quadratwurzeln, eintreten lassen kann.

b) Anstatt zu $\left[r_{1}\right]$ direct eine Gruppe $\left[s_{1}\right]$ zu suchen, für welche $K_{\tau_{1}, s_{1}} \equiv 1$, kann man auch indirect verfahren und dadurch die Zurückfübrung der Gruppe von $Z$ Substitutionen auf die von nur $Z_{1}$ noch einfacber gestalteu.

Sei, um die Begriffe zu fixiren, $p$ zunächst von der Form $4 n$ oder $4 n-1$. Von der Gleichung, welche die $2^{2 p}-1$ Gruppen bestimmt, sei wieder eine Wurzel, $\left[r_{1}\right]$, gesucht. Wir behaupten nun: Hat man weiter diejenige Gleichung des Grades $R_{p-1}$ vollständig aufgelöst, welche die $R_{p-1}$ Zerlegungen

$$
\left[r_{1}\right] \equiv\left(\alpha_{1}\right),\left(\beta_{1}^{\prime}\right) \equiv\left(\alpha_{2}\right),\left(\beta_{2}\right) \equiv \cdots \equiv\left(\alpha_{R_{p-1}}\right),\left(\beta_{R_{p-1}}\right)
$$

von $\left[r_{1}\right]$ in Paare $\left(\alpha_{t}\right),\left(\beta_{l}\right)$ ungerader Charakteristiken liefert, und endlich durch Auflösen von $2 p-1$ quadratischen Gleichungen gewisse $2 p-1$ dieser Paare in ihre beiden Elemente $\left(\alpha_{t}\right)$ und $\left(\beta_{\imath}\right)$ getrennt, so ist die vollständige Lösung der vorgegebenen Gleichnng bewirht.

Denn eine hinzugefügte Gruppe $\left[s_{1}\right]$, wo $K_{r_{1}, s_{1}} \equiv 1$, würde nur die Trennung der $R_{p-1}$ Charakteristiken $\left(\alpha_{\iota}\right)$ von den $\left(\beta_{\iota}\right)$ leisten. Aber bildet man aus solchen $R_{p-1}$ Grössen $\left(\alpha_{\iota}\right)$ eines unserer Systeme (\$ 7.) 
von $2 p-1$ Charakteristiken, für welches die Sumne je dreier gerade wird:

$$
\left(\alpha_{1}\right),\left(\alpha_{2}\right), \cdots,\left(\alpha_{z_{p-1}}\right),
$$

so geht dieses System in ein ganz gleich defnirtes über, wenn mau irgend welche der $\left(\alpha_{t}\right)$ durch die zugehörigen $\left(\beta_{t}\right)=\left(r_{1} \alpha_{t}\right)$ ersetzt, da nach XVIII.

$$
\left(\alpha_{1} \alpha_{z} \alpha_{\lambda}\right) \text { und }\left(r_{1} \alpha_{t} \alpha \alpha_{\lambda}\right)
$$

zugleich gerade und ungerade sind. Man hat also nur aus den $R_{p-1}$ Paaren vou $\left[r_{2}\right] 2 p-1$ solche Paare,

$$
\left(\alpha_{1}\right),\left(\beta_{1}\right) ;\left(\alpha_{2}\right),\left(\beta_{2}\right) ; \cdots ;\left(\alpha_{2 p-1}\right),\left(\beta_{2 p-1}\right),
$$

auszuwählen, für welche die Summe von je ä Charakteristikeu, aus dreien der Paare genommen, immer gerade ist. Irgeud $2 p-1$ dieser Charakteristiken, genommen aus allen $2 p-1$ Paaren, bilden dann ein System, wie es in $\$ 7$. betrachtet ist: nämlich es existirt eine weitere zugehörige Gruppe $\left[s_{1}\right]$, welche diese $2 p-1$ Charakteristikeu enthält, und aus den Zusammensetzungen von $\left[r_{1}\right],\left[s_{1}\right]$ und den $2 p-1$ Charakteristikeu folgen, nach XXV. und XX., alle Gruppen und Charakteristiken eindeutig. Um ein solches System von $2 p-1$ Charakteristiken aber selbst eindeutig darzustellen, hat man, nachdem die $2 p-1$ Paare

$$
\left(\alpha_{1}\right),\left(\beta_{1}\right) ; \cdots ;\left(\alpha_{z p-1}\right),\left(\beta_{2 p-1}\right)
$$

durch die Auflösung der Gleichung vom Grade $R_{p-1}$, welche alle $R_{p-1}$ Paare von $\left[r_{1}\right]$ liefert, bekannt gewordell sind, wur noch diese $2 p-1$ Paare durch ebenso viele quadratische Gleichungen in ihre beiden Bestandtheile zu zerlegen.

Die hier vollständig aufzulösende Gleichung vom Grade $R_{p-1}$ ist nun eine solche mit $Z_{1}$ Substitutionen, und kann selbst nach der für die ursprüngliche Gleichung mit $Z$ Substitutionen gegebenen Metbode weiter behandelt werden. In der That kann man nach dem Früheren die $R_{p-1}$ Paare von $\left[r_{1}\right]$ eindeutig den $R_{p-1}$ ungeraden $(p-1)$ reihigen Charakteristiken zuordnen; nämlich so, dass irgend drei solchen $(p-1)$ reihigen Charakteristiken $\left(\alpha_{\imath}^{\prime}\right) ;\left(\alpha_{x}^{\prime}\right) ;\left(\alpha_{i}^{\prime}\right)$, für welche. $\left(\alpha_{1}^{\prime} \alpha_{x}^{\prime} \alpha_{i}^{\prime}\right)$ gerade ist, drei solche Zerlegungen von $\left[r_{1}\right]$

$$
\left(\alpha_{t}\right),\left(\beta_{t}\right) ;\left(\alpha_{x}\right),\left(\beta_{x}\right) ;\left(\alpha_{2}\right),\left(\beta_{k}\right)
$$

entsprechen, für welche

$$
\left(\alpha_{1} \alpha_{x} \alpha_{i}\right),\left(\alpha_{2} \alpha_{x} \beta_{2}\right) \text {, etc. }
$$

gerade wird. - Die Gleichung vom Grade $R_{p-1}$ wird also dadurch aufgelöst, dass man zunächst eine Wurzel der Gleichung von Grade $2^{2(p-1)}-1$ sucht, welche die $2^{2(p-1)}-1$ Gruppes bestimmt, die alas je $h_{p-2}$ Paaren der $\left(a_{\ell}^{\prime}\right)$, d. h. aus je $R_{p-2}$ Paaren vou Zerlegungen der Art

$$
\left(\alpha_{t}\right),\left(\beta_{\imath}\right) ;\left(\alpha_{x}\right),\left(\beta_{x}\right),
$$


bestehen; dass man sodam die Gleichung rom Grade $R_{p-2}$ mit $Z_{2}$ Substitutionen vollständig und endlich $2 p-3$ quadratische Gleichungen löst.

Dasselbe, mit Vertauschung von Gerade und Ungerade und von $k_{u}$ mit $S_{\mu}$, gilt für $p=4 n-2$ und $p=4 n-3$. Indem man also dieses Verfahren fortsetzt, ergeben sich überhaupt zur Lösung der vorgelegten Gleichung folgende Operationen: Man hat der Reille nach $z u$ suchen je eine Wurzel von Gleichungen der Grade

$$
2^{2 p}-1,2^{2(p-1)}-1, \cdots, 2^{6}-1 \text {, }
$$

- Gleichungen, die bez. bei den $p$-, p-1-, , , 3-reihigen Charakteristiken auftreten -, sodann eine (bei den 2 -reiliggen Charakteristiken auftretende) allgemeine Gleichung $6^{\text {ten }}$ Grades vollstïndig, und endlich

$$
5+7+9+\cdots+(2 p-1)=p^{2}-4
$$

quadratische Gleichungen zu lösen.

c) Auch dieses Verfahren b) kann noch modificirt werden. Man kann nämlich auch die erste Gruppe $\left[r_{1}\right]$ indirect bestimmen, indem man zunächst eine Wurzel der Gleichung vom Grade $R_{p}$ sucht, welche die Zuorduung der $R_{p}$ ungeraden Charakteristiken liefert. Von der übrigbleibenden Gleichung vom Grade $R_{p}-1$ bestimmt man dann wiederum eine Wurzel, was mit der ersten Wurzel verbunden ein Paar von $\left[r_{1}\right]$ liefert; um die ïbrigen Charakteristiken sämmtlich eindeutig zu finden, hat man dann noch eine Gleichung vom Grade $R_{p-1}-1$, welche die übrigen Paare von $\left[r_{1}\right]$ liefert, vollständig, und $2 p-2$ quadratische Gleichungen zu lösen. - Die Gleichung vom Grade $R_{p-1}-1$, die zu den Gleichungen der $(p-1)$-reihigen Charakteristiken dieselbe Beziehung hat, wie die obige vom Grade $R_{p}-1$ zu denen der $p$-reihigen Charakteristiken, wird nun nach derselben Methode weiter behandelt; man hat also zur Lösung der vorgelegten Gleichung die Operationen auszuführen: $E s$ ist der Reilhe nach zu suchen je eine Wurzel von Gleichungen der Grade

$$
R_{p}, R_{p}-1, R_{p-1}-1, R_{p-2}-1, \cdots, R_{3}-1,
$$

- Gleichungen, von denen die ersten beiden bei den $p$-reihigen, die ïbrigen bez. bei den $p-1-, p-2-, \ldots, 3$-reitigen Charaliteristiken auftreten -; sodann eine allgemeine Gleichung des $5^{\text {ten }}$ Grades vollständig, und endlich

$$
4+6+8+\cdots+(2 p-2)=(p+1)(p-2)
$$

quadratische Gleichungen zu lösen.

Stellt man dieselbe Betrachtung für die geraden Charakteristiken an, so zeigt sich, dass die Lösung auch so geschehen kann: Es ist der Reihe nach zu suchen je eine Wurzel von Gleichungen der Grade

$$
S_{p}, S_{p}-1, S_{p-1}-1, S_{p-2}-1, \cdots, S_{3}-1, S_{2}-1 \text {, }
$$


- von denen die ersten beiden bei den p-reihigen, die ïbrigen bez. bei den $p-1-, p-2-, \ldots, 3-, 2$-reikigen Charakteristiken auftreten -; sodann sind noch

$$
1+2+4+6+\cdots+(2 p-2)=p(p-1)+1
$$

quadratische Gleichungen zu lösen.

2. a) Ein zweites Verfahren zur Lösung der vorgelegten Gleichungen besteht darin, dass man dieselben auf diejenige Gleichung des Grades $2 p+1$ zurückführt, welche den $2 p+1$ Charakteristiken eines ,ausgezeichneten" Systems zugeordnet ist. Diese Gleichung hat noch zur Gruppe die allgeneinste, welche bei einer Gleichung $(2 p+1)^{\text {ten }}$ Grades überhaupt existirt; denn unsere Substitutionen erlauben, sämmtliche $2 p+1$ Charakteristiken eines ausgezeichneten Systems beliebig zu permutiren. Die Ordnung dieser Substitutionsgruppe beträgt also wenigstens

$$
1 \cdot 2 \cdot 3 \cdots(2 p+1)
$$

und auch nicht mehr, da sie $(\S 5$., 3. und $\S 10 ., 1$.

werden muss.

$$
=\frac{Z}{L_{0}}=1 \cdot 2 \cdot 3 \cdots(2 p+1)
$$

$\mathrm{Zu}$ dieser Zurückführung ist also nur nöthig, eine Wurzel einer Gleichung vom Grade

$$
L_{0}=\frac{R_{2 p}}{1 \cdot 2 \cdot \frac{R_{2(p-1)}}{3} \cdots R_{2}}
$$

zu suchen, welche die $L_{0}$ ausgezeichneten Systeme bestimmt. Diese Systeme zerfallen aber für $p=4 n$ oder $4 n-1$ in $S_{p}$ Classen, den $S_{p}$ verschiedenerı geraden Summen entsprechend; und für $p=4 n+1$ oder $4 n+2$ in $R_{p}$ Classen, den $R_{p}$ verschiedenen ungeraden Summen entsprechend. Also werden die zur Lösung nöthigen Operationen: Man sucht a) für $p=4 n, 4 n-1$ : eine Wurzel einer Gleichung vom Grade $S_{p}$, sodann eine Wurzel einer Gleichung vom Grade $\left.\frac{L_{0}}{S_{p}} ; \beta\right)$ für $p=4 n+1,4 n+2$ : eine Wurzel einer Gleichung vom Grade $R_{p}$, sodann eine Wurzel einer Gleichung vom Grade $\frac{L_{0}}{R_{p}}$; endlich hat man in beiden Fällen eine allgemeine Gleichung des Grades $2 p+1$ vollständig zu lösen.

b) Durch eine Modification dieses Verfahrens fülırt man die vorgelegte Gleichung auf eine allgemeine Gleichung $(2 p+2)^{\text {tex }}$ Grades zurück.

Für gerades $p$ sucht man eine Wurzel*) der Gleichung vom Grade

-) Die Adjunction dieser eiven Wurzel charakterisirt den Fall, der bei der Zweitheilung der Perioden der hyperelliptisehen Functionen eintritt. 
$\frac{L_{0}}{2 p+2}$, welche die $\frac{L_{0}}{2 p+2}$,vollständigen" "Systeme nit Summe 0 bestimmt. Man komnt danu auf die Gleichung vom Grade $2 p+2$, welche den $2 p+2$ Charakteristiken eines solchen Systems zugeordnet ist.

Für ungerade $p$ ist es möglich, die ausgezeichneten Systeme von $2 p+1$ Charakteristiken mit gleicher Summe $(\alpha)$ zu je $2 p+2$ zu gruppiren. Sei ein solches System

$$
(\alpha) \equiv\left(\alpha_{1}\right),\left(\alpha_{2}\right), \cdots,\left(\alpha_{2 p+1}\right),
$$

so kaun man durch die Substitutionen $\left\{\alpha \alpha_{t}\right\}$ daraus die weiteren $2 p+1$ Systeme ableiten:

$$
\begin{aligned}
(\alpha) & =\left(\alpha_{1}\right),\left(\alpha \alpha_{1} \alpha_{2}\right),\left(\alpha \alpha_{1} \alpha_{3}\right), \cdots,\left(\alpha \alpha_{1} \alpha_{2 p+1}\right), \\
& \equiv \cdots, \\
& \equiv\left(\alpha \alpha_{2 p+1} \alpha_{1}\right),\left(\alpha \alpha_{2 p+1} \alpha_{2}\right), \cdots,\left(\alpha \alpha_{2 p+1} \alpha_{2 p}\right),\left(\alpha_{2 p+1}\right) .
\end{aligned}
$$

Macht man die analogen Substitutionen auf eines dieser Systeme, also die Substitutionen $\left\{\alpha_{\imath} \alpha_{z}\right\},\left\{\alpha \alpha_{t}\right\}$, so erhält man nur immer wieder dieselben $2 p+2$ Systeme. Die aus diesen Substitutionen abgeleitete Gruppe bestebt aus der Gruppe ron der Ordnung $1 \cdot 2 \cdot 3 \cdots(2 p+1)$, welche aus den Transpositionen $\left\{\alpha_{1} \alpha_{x}\right\}$ abgeleitet ist, verbunden mit den $2 p+2$ Substitutionen $1,\left\{\alpha \alpha_{\imath}\right\}$, und wird von der Ordnung $1 \cdot 2 \cdot 3 \cdots(2 p+2)$. Die Gleichung, welche solchen $2 p+2$ Systemen zugeordnet ist, ist daher eine allgemeine vom $(2 p+2)^{\text {ten }}$ Grade. Um die vorgelegrte Gleichung auf dieselbe zurückzuführen, bat man eine Wurzel*) der Gleichung vom Grade $\frac{L_{0}}{2 p+2}$ zu suchen, welche die $\frac{L_{0}}{2 p+2}$ Gruppen von je $2 p+2$ solchen Systemen bestimmt. Statt dessen kann man wieder für $p=4 n+1[4 n-1]$ zuerst eine Wurzel einer Gleichung vom Grade $R_{p}\left[S_{p}\right]$, sodann eine Wurzel einer Gleichung rom Grade $\frac{L_{0}}{(2 p+2) R_{p}}\left[\frac{I_{0}}{(2 p+2) S_{p}}\right]$ aufsuchen.

3. Die beiden Methoden 1. und 2. lassen sich mit einander combiniren. So gelangt man bei dem Verfabren 1. b), indem man je eine Wurzel von Gleichungen der Grade

$$
2^{2 p}-1,2^{2(p-1)}-1, \cdots, 2^{2(p-\mu)}-1
$$

sucht, auf eine Gleichung vom Grade $R_{p-\mu-1}$, welche den $R_{p-\mu-1}$ ungeraden $(p-\mu-1)$-reihigen Charakteristiken entspricht, und hat nach deren vollständiger Lösung noch

$$
\begin{gathered}
(2 p-2 \mu-1)+(2 p-2 \mu+1)+\cdots+(2 p-1) \\
=(\mu+1)(2 p-\mu-1)
\end{gathered}
$$

quadratische Gleichungen zu lösen. Die Lösung der Gleichung vom Grade $R_{p-\mu-1}$ kaun aber nach dem Verfahren 2. bewerkstelligt werden.

*) Für die Adjunction dieser Wurzel gilt die Anmerkung der vorhergehenden Seite. 


\section{Abschnitt.}

Das Additionstheorem der Thetafunctionen.

\section{$\S 12$.}

\section{Die Thetafunctionen.}

Wir stellen in diesem Paragraphen diejenigen Bezeichnuugen und bekannten Beziehungen, von welchen im Folgenden Gebrauch gemacht wird, zusammen (s. d. Ann. XIV, p. 280).

Die Thetafunction, von der wir ausgehen, wird definirt durch

$$
\vartheta_{0}(u)=\vartheta_{0}\left(u_{1}, u_{2}, \cdots, u_{p}\right)=\sum e^{\sum_{a, b} v_{a} v_{b} a_{a, b}+{ }_{a}^{2} \sum_{a} u_{a}},
$$

wo die Summationen in Exponenten von $e$ auf die Werthe $1,2, \cdots, y$ der Indices $a, b$, die Summation $\sum$ auf alle positiven und negativen ganzzahligen Werthe der $p$ Grössen $v_{1}, v_{2}, \cdots, v_{p}$ zu erstrecken ist. Die Moduln $a_{a, b}$ der f'unction haben die Bedingung

$$
a_{a, b}=a_{b, a}
$$

zu erfüllen, feruer die Bedingung der Convergenz der $\vartheta$-Reihe - dass nämlich der reelle Theil von $\sum_{a, b} v_{\mathfrak{a}} \nu_{\mathfrak{b}} a_{a, b}$ eine wesentlich negative quadratische Form sein muss -; im Uebrigen aber seien diese $\frac{1}{2} p(p+1)$ Grössen ganz beliebig gelassen.

Den $2^{2 p}-1$ Gruppencharakteristiken $[\alpha]$ :

$$
[\alpha]=\left[\begin{array}{cccc}
n_{1}^{\alpha} & n_{2}^{\alpha} & \cdots & n_{p}^{\alpha} \\
m_{1}^{\alpha} & m_{2}^{\alpha} & \cdots & m_{p}^{\alpha}
\end{array}\right]
$$

werden bez. Systeme halber Perioden zugeordnet:

$$
\frac{1}{2} \widetilde{\omega}_{a}^{\alpha}=\frac{1}{2} m_{a}^{\alpha} \pi i+\frac{1}{2} \sum_{b=1}^{\mathfrak{b}=p} n_{b}^{\alpha} a_{a, b}, \quad(\mathfrak{a}=1,2, \cdots, p)
$$

die kurz mit $\frac{1}{2} \varpi^{\alpha}$ bezeichnet werden. Und den eigentlichen Charakteristiken $(0)$ und $(\alpha 0)=(\alpha)$ entsprechend, mögen die allgemeinen Thetafunctionen definirt sein durch den obigen Ausdruck $\vartheta_{0}(u)$ und durch $\vartheta_{v \alpha}(u)=\vartheta_{\alpha}(u)$, wo:

$$
\mathfrak{\vartheta}_{\alpha}(u)=e^{\frac{1}{4} \sum_{a, b} n_{\alpha}^{\alpha} n_{b}^{\alpha} a_{a, b}+\frac{1}{2} \pi i \sum_{a} n_{a}^{\alpha} m_{a}^{\alpha}+\sum_{a} n_{a}^{\alpha} u_{a}} \cdot \vartheta_{0}\left(u+\frac{1}{2} \varpi^{\alpha}\right) .
$$


Bei dieser Zuordnung der halben Perioden $\frac{1}{2} \widetilde{\varpi}^{\alpha}$ zur Gruppencharakteristik $\lfloor\alpha\rfloor$ und von $\vartheta_{a}(u)$ zur eigentlichen Charakteristik $(\alpha)$ denken wir uns die Elemente $n_{\alpha}^{\alpha}$ und $m_{\alpha}^{\alpha}$ von $[\alpha]$ und $(\alpha)$ nicht auf ihre Werthe 0 oder 1 (mod. 2) reducirt. Vielmehr ist:

$$
\vartheta_{a, \beta p}(u)=(-1)^{\sum n_{a} m_{a}^{\beta}} \cdot \vartheta_{\alpha}(u),
$$

und wir versteben daher in diesem Abschnitt, um Weitlïufigkeiten zu vermeiden, unter $(\alpha \beta)$ immer eine Charakteristik, deren Elemente die algebraischen Summen entsprechender Elemente von $(\alpha)$ und $(\beta)$ sind. Insbesondere ist unter $\mathfrak{\vartheta}_{0}(u)$ verstanden, dass die Elemente von $(0)$ gleich 0 sind. Die $\vartheta$-Funetionen zerfallen in zwei Classen: in gerade und ungerade, je nachdem $(\alpha)$ gerade oder ungerade ist; die halben Perioden $\frac{1}{2} \varpi^{\alpha}$ bilden nur eine zusammenhängende Classe.

Man hat:

$$
\begin{gathered}
\vartheta_{\alpha}\left(-u_{1},-u_{2}, \cdots,-u_{p}\right)=(-1)^{\Sigma n^{\alpha} m^{\alpha}} \cdot \vartheta_{\alpha}\left(u_{1}, u_{2}, \cdots, u_{p}\right), \\
\vartheta_{\alpha}\left(u+\varpi^{\beta}\right)=(-1)^{\sum\left(n^{\alpha} m^{\beta}+n^{\beta} m^{\alpha}\right)} \cdot e^{-\sum_{a, b} n_{a}^{\beta} n_{b}^{\beta} a_{a, b}-2 \sum_{\alpha} n_{a}^{\beta} u_{a}} \cdot \vartheta_{\alpha}(u), \\
\vartheta_{\alpha}\left(u+\frac{1}{2} \varpi s^{\beta}\right)=i^{-\sum n^{\beta} m^{\alpha, \beta}} \cdot e^{-\frac{1}{\alpha} \sum_{a, b} n_{a}^{\beta} n_{b}^{\beta} a_{a, b}-\sum_{a} n_{a}^{\beta} u_{a}} \cdot \vartheta_{\alpha \beta}(u),
\end{gathered}
$$

wo wieder

gesetzt ist.

$$
\sum n^{\gamma} m^{\delta} \text { für } \sum_{\mathfrak{a}} n_{\mathfrak{a}}^{\gamma} m_{\mathfrak{a}}^{\delta}
$$

Ferner ist

$$
\boldsymbol{\vartheta}_{\alpha}\left(\frac{1}{2} \varpi^{\beta}\right)=0,
$$

wemu $(\alpha \beta)$ eine ungerade Charakteristik ist.

Endlich benutzen wir das Theorem: dass zwischen je $2^{p}+1$ Producten der Form

$$
\vartheta_{\varepsilon}\left(u+v^{\prime}\right) \cdot \vartheta_{\varepsilon}\left(u+v^{\prime \prime}\right),
$$

mit verschiedenen Indices $\varepsilon$, aber gleichen Argumenten $u_{a}$ und gleichen Summen $v_{a}^{\prime}+v_{a}^{\prime \prime}$, eine homogene lineare Relation stattfindet, deren Coefficienten von den $u_{a}$ unabhängig sind.

\$ 13.

Systeme von $2^{p}$ Indices für $p \geqq 3$. Product

Nach dem letzten Satze des vorigen Paragraphen kaun man das 


$$
\vartheta_{0}(u+v+w) \vartheta_{0}(u-v)
$$

linear und homogen durch $2^{p}$ Producte der Form

$$
\vartheta
$$

darstellen, wenn man hier $2^{p}$ verschiedene Indices $\varepsilon$ derart wählt, dass zwischen den $2^{p}$ Producten selbst keine identische lineare Relation besteht. Wir wollen nun für $p \geqq 3$ zeigen, wie ein solches System von $2^{p}$ Indices zu wählen ist, damit die Bestimmung der von deu $u_{\alpha}$ unabhängigen Coefficienten des gesuchten Ausdrucks möglichst einfach wird.

Sei

$$
\left(\alpha_{0}\right) \equiv\left(\alpha_{1}\right),\left(\alpha_{2}\right), \cdots,\left(\alpha_{2 p+1}\right)
$$

irgend eines der in $\S 8$. behandelten ,ausgezeichneten "Systeme vou $2 p+1$ Charakteristiken; für welches also die $\left(\alpha_{t} \alpha_{x} \alpha_{2}\right)$ von entgegeugesetztem Charakter sind, wie die $\left(\alpha_{\iota}\right)$, für $\iota \gtrless * \gtrless \lambda$, von $1,2, \cdots, 2 p+1$.

Man nehme aus diesem System, für $p \geqq 3$, die Charakteristiken

$$
\left(\alpha_{0}\right),\left(\alpha_{1}\right),\left(\alpha_{2}\right), \cdots,\left(\alpha_{7}\right)
$$

heraus und verbinde die äbrigen $2 p-6$ beliebig $2 u p-3$ Paaren:

$$
\left[u_{8} \alpha_{9}\right]=\left[l_{1}\right], \quad\left[\alpha_{10} \alpha_{11}\right]=\left[l_{2}\right], \cdots,\left[\alpha_{2 p} \alpha_{2 p+1}\right]=\left[l_{p-s}\right] .
$$

Seien danu weiter mit

$$
\left[\lambda_{1}\right],\left[\lambda_{2}\right],\left[\lambda_{3}\right], \cdots,\left[\lambda_{2} p-3\right]
$$

alle $2^{y-3}$ Combinationen dieser $p-3$ Grössen $\left[l_{1}\right],\left[l_{2}\right], \cdots\left[l_{p-3}\right]$ zu $0,1,2, \cdots, p-3$ bezeichnet, so hat man in

$$
\left\{\begin{array}{l}
\left(\lambda_{j} \alpha_{0}\right),\left(\lambda, \alpha_{3}\right), \cdots,\left(\lambda_{j} \alpha_{7}\right), \\
\left(j=1,2, \cdots, 2^{p-3}\right)
\end{array}\right.
$$

ein System ron $2^{p}$ Charakteristiken, dem man ein zweites analoges System zuordnen kann, das mit dem ersteren in besonderer Beziehung steht.

Sei nämlich eine neue Charakteristik $(l)$ eingeführt durch:

$$
\begin{aligned}
& (l)=\left(\alpha_{8} \alpha_{10} \cdots \alpha_{2 p}\right), \text { für gerade } p, \\
& (l)=\left(\alpha_{0} \alpha_{8} \alpha_{10} \cdots \alpha_{2 p}\right), \text { für ungerade } p .
\end{aligned}
$$

Danu sind, nach den Eigenschaften der ausgezeichneten Systeme, für jedes $p$ :

$$
\begin{array}{ll}
\text { alle }(l \lambda,) \text { gerade, } & \text { für } j=1,2,3, \cdots, 2^{p-3}, \\
\text { alle }\left(l \lambda_{j} \alpha_{\iota} \alpha_{x}\right) \text { ungerade, für } \iota \gtrless x, \text { von } 0,1, \cdots, 7 .
\end{array}
$$

Bildet man daher ein zweites System von $2^{p}$ Charakteristiken: 


$$
\left\{\begin{array}{c}
{\left[i \lambda_{j^{\prime}} \alpha_{0}\right],\left[l \lambda_{j} \alpha_{1}\right], \ldots,\left[l \lambda_{j^{\prime}} \alpha_{7}\right]} \\
\left(j^{\prime}=1,2, \ldots, 2^{p-3}\right)
\end{array}\right.
$$

so hat dieses zum ersten die Beziehung: Addirt man irgend eine Charakteristik des einen Systems zu allen $2^{p}$ Charakteristiken des andern Systems, so sind immer von den $2^{p}$ Summen nur $2^{p-3}$ gerade, die übrigen $7 \cdot 2^{p-3}$ ungerade. Und zwar hat man $2^{p-3}$ verschiedene Grössen des einen Systems mit denselben $2^{p-3}$ Grössen des andern Systems $z u$ verbinden, um gerade Summen zu erhalten.

Man hat nämlich irgend eine der $2^{p-3}$ Grössen $\left(l \lambda_{j^{\prime}} \alpha_{t}\right)$, für $j^{\prime}=1,2, \cdots, 2^{p-3}$, zu verbinden mit den $2^{p-3}$ Grössen $\left(\lambda_{j} \alpha_{\iota}\right)$, für $j=1,2, \ldots, 2^{p-3}$, um gerade Summen zu erhalten; dagegen mit $\left(\lambda_{j} \alpha_{*}\right)$, wo $x \gtrless \iota$, um ungerade Summen zu erhalten.

Die Beziehung zwischen beiden Systemen würde offenbar bestehen bleiben, wenn man zu jeder Charakteristik jedes der beiden Systeme irgend eine, aber immer dieselbe Charakteristik hinzuaddirte. Addirt man $\left(\alpha_{0}\right)$ und beachtet die Darstellang eines ausgezeichneten Systems, wie sie am Ende des $\$ 8$. gegeben ist, so kann man zwei einander zugehörige Systeme von je $2^{p}$ Charakteristiken, welche in der obigen Beziehung stehen, auch so darstellen (indem man an Stelle der $\left[l_{2}\right]$ die dortigen $\left[t_{i}\right]$ setzt).

Seien mit $\left[t_{j}^{\prime}\right]$ alle Combinationen der $p-3$ Grösseu $\left[t_{p-3}\right]$, $\left[t_{p-4}\right], \cdots,\left[t_{1}\right]$, zu $0,1, \cdots, p-3$ genommen, bezeichnet, so ist das erste Systèm

$$
\begin{array}{cl}
{\left[t_{j}^{\prime}\right],\left[t_{j}^{\prime} t_{p} t_{p-1} \cdots t_{1}\right],\left[t_{j}^{\prime} r_{p} t_{p-1} t_{p-2} \cdots t_{1}\right],} & {\left[t_{j}^{\prime} r_{p-1} t_{p-2} t_{p-3} \cdots t_{1}\right],} \\
& {\left[t_{j}^{\prime} r_{p-2} t_{p-3} \cdots t_{1}\right],} \\
{\left[t_{j}^{\prime} s_{p} t_{p-1} t_{p-2} \cdots t_{1}\right],} & {\left[t_{j}^{\prime} s_{p-1} t_{p-2} t_{p-3} \cdots t_{1}\right],} \\
& {\left[t_{j}^{\prime} s_{p-2} t_{p-3} \cdots t_{1}\right] ;}
\end{array}
$$

und das zweite entsteht aus diesem, indem man zu jeder seiner Charakteristiken

addirt.

$$
(l)=\left(g r_{1} r_{2} \cdots r_{p-8} t_{p-2} t_{p}\right)
$$

Endlich kaun man zwei Systeme dieser Art auch so darstellen: Sei

$$
\left(\beta_{0}\right) \equiv\left(\beta_{1}\right),\left(\beta_{9}\right), \cdots,\left(\beta_{7}\right)
$$

ein 7-System ungerader Charakteristiken, mit gerader Summe $\left(\beta_{0}\right)$, enthalten in den $p-3$ Gruppenpaaren:

$$
\left[r_{1}\right],\left[s_{1}\right] ; \cdots ;\left\lceil r_{p-3}\right\rfloor,\left[s_{p-3}\right] ;
$$

und $\left[t_{j}^{\prime}\right]$ wieder alle Combinationen der Grössen $\left[t_{i}\right]=\left[r_{i} s_{t}\right]$. Das erste System ist dann 


$$
\left(t_{j}^{\prime} \beta_{0}\right),\left(t_{j}^{\prime} \beta_{1}\right), \cdots,\left(t_{j}^{\prime} \beta_{7}\right) ; \quad\left(j=1,2, \cdots, 2^{p-s}\right),
$$

das zweite entsteht aus diesem durch Addition von

$$
\left(\beta_{0} r_{1} r_{2} \cdots r_{p-s}\right)
$$

zu allen seinen Charakteristiken.

Die bezeichnete Beziehung zwischen zwei solchen einander zugehörigen Systemen macht die Einführung eines derselben an Stelle der $2^{p}$ Indices $\varepsilon$ der am Arfang dieses Paragraphen angegebenen Relation vortheilhaft. Wir benutzen zu diesem Zwecke die zuerst gegebene Form (A), (A') der beiden Systeme.

\section{$\S 14$.}

Das Additionstheorem für $p \geqq 3$.

Nach den Bemerkungen des vorigen Paragraphen nehmen wir die Relation an:

$$
\begin{aligned}
& \vartheta_{0}(u+v+w) \vartheta_{0}(u-v)=\sum_{\rho} \sum_{j} c_{v}^{(j)} \vartheta_{j_{j} u_{e}}(u) \cdot \vartheta_{\lambda_{j} u_{e}}(u+w), \\
& \left(\begin{array}{l}
\varrho=0,1, \cdots, 7, \\
j=1,2, \cdots, 2^{p-3}
\end{array}\right),
\end{aligned}
$$

worin die noch $\mathrm{zu}$ bestimmenden Grössen $\ell_{p}^{(j)}$ nur van den $v_{a}$ und $w_{\mathfrak{a}}$ abhäugen. Setzt man für die $u_{a}$ halbe Perioden ein, entsprechend den Charakteristiken $\left(A^{\prime}\right)$ des $\$ 13$.:

$$
u_{a}=\frac{1}{2} \widetilde{w}_{a}^{i 2, \alpha} e \text {, }
$$

so ergiebt sich zur Bestimmung der $c_{e}^{(i)}$ das Gleichungssystem

$$
\begin{aligned}
& \vartheta_{u}\left(\frac{1}{2} \widetilde{\varpi}^{l \lambda_{j} \alpha_{\varphi}}+v+w\right) \cdot \vartheta_{u}\left(\frac{1}{2} \widetilde{\omega}^{l \lambda_{j} u_{u}}-v\right)= \\
& =\sum c_{\rho}^{(j)} \vartheta_{\lambda_{j} \alpha_{e}}\left(\frac{1}{2} \varpi^{b \lambda_{j} \alpha_{i}}\right) \cdot \vartheta_{\lambda_{j} \alpha_{e}}\left(\frac{1}{2} \varpi^{l \lambda_{j^{\prime}} \alpha_{\rho}}+w\right),
\end{aligned}
$$

oder, nach Reduction nit Hülfe der Formeln des $\$ 12$.

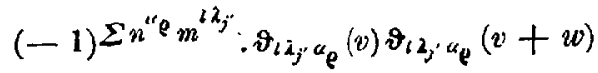

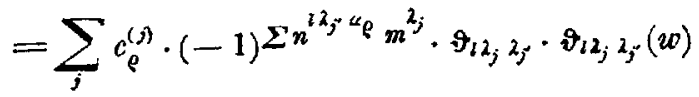

$$
\begin{aligned}
& \left(j^{\prime}=1,2, \cdots, 2^{p-3}\right) .
\end{aligned}
$$

Man hat in (2) $2^{p-3}$ Gleichungen, welche solche $2^{p-3}$ Grössen $\epsilon_{c}^{(j)}$, die gleiches $\varrho$, aber verschiedenes $j$, für $j=1,2, \cdots, 2^{p-3}$, haben, gesondert von den übrigen Grössen bestimmen. Um aber diese Gilej- 
chungen, insbesondere ibre Determinante, in übersichtlicher Weise weiter behandeln zu können, wird es nöthig, zuerst die Indices $\lambda_{j}$ in besonderer Weise zu ordnen und damn statt der $e_{\rho}^{(j)}$ andere Grössen einzuführen.

Die $\lambda_{j}$ sollen so geordnet werden:

Zunächst werde $\lambda_{1}=0, \lambda_{2}=l_{1}$ gesetzt; diese beiden werden dann mit $l_{2}$ zusarmmengesetzt; sodann die so gebildeten $2^{2}$ mit $l_{3}$; sodann die so gebildeten $2^{3}$ mit $l_{4}$ zusammengesetzt; etc.; also:

$\lambda_{1}=0$

$\lambda_{2}=l_{1}$

$\lambda_{3}=l_{2}, \lambda_{4}=l_{1} l_{2}$

$\lambda_{5}=l_{3}, \lambda_{6}=l_{1} l_{3}, \lambda_{i}=l_{2} l_{3}, \lambda_{8}=l_{1} l_{2} l_{3} ;$

$\lambda_{9}=l_{4}, \lambda_{10}=l_{1} l_{4}, \lambda_{11}=l_{2} l_{4}, \lambda_{12}=l_{1} l_{2} l_{4}, \lambda_{13}=l_{3} l_{4}, \quad \lambda_{14}=l_{1} l_{3} l_{4}$,

etc.$$
\lambda_{15}=l_{2} l_{3} l_{1}, \lambda_{16}=l_{1} l_{2} l_{3} l_{4}
$$

Allgemein ist:

$$
\lambda_{k+2^{k-1}}=\lambda_{k} \lambda_{2^{k-1}+1},
$$

für

$$
h=j \cdot 2^{k}+1, j \cdot 2^{k}+2, \ldots, j \cdot 2^{k}+2^{k-1},
$$

wo $j=0,1,2, \cdots$ zu setzen ist und $z$ jeden Werth $1,2,3 \ldots$ annehmen darf, wenn man noch

festsetzt.

$$
\lambda_{h^{\prime}}=\lambda_{h}, \text { für } h^{\prime} \equiv h\left(\bmod .2^{p-3}\right)
$$

Hiernach ist zunächst:

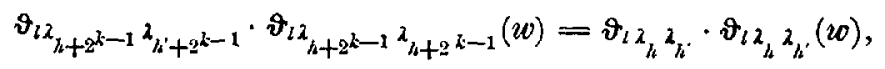

wenn $h$ und $h$ wie $h$ in (3) gehen.

Ferner sei gesetzt

$$
(-1)^{\sum \frac{1}{2} n^{l_{k}} m^{l_{k}}}=i_{l_{k}},
$$

für

$$
k=1,2, \cdots, p-3,
$$

und weiter definirt:

$$
i_{l_{k} k_{k^{\prime}}}=i_{l_{k}} \cdot i_{k_{k^{\prime}}} \cdot(-1)^{\sum n^{l_{k} m^{l_{k}}}} .
$$

Aus der obigen Darstellung der $\lambda_{j}$ durch die $l_{\mathrm{s}}$ folgt dann sogleich die Definition der Grössen $i_{\lambda_{j}}$. Beachtet man, dass

$$
\sum\left(n^{k_{k}} m^{z_{k}}+n^{k^{k}} m^{l_{k}}\right)=K_{l_{k} l_{k^{\prime}}} \equiv 0(\bmod .2),
$$


(weil z. B. die beiden in $\left[l_{1}\right]=\left[\alpha_{s} \alpha_{9}\right]$ gepaart enthaltenen Charakteristiken $\left(\alpha_{\delta}\right),\left(\alpha_{4}\right)$ in $\left[l_{2}\right]$ nicht vorkommen), also auch

so folgt ans (5):

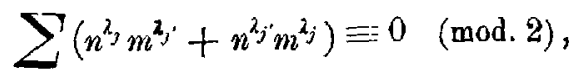

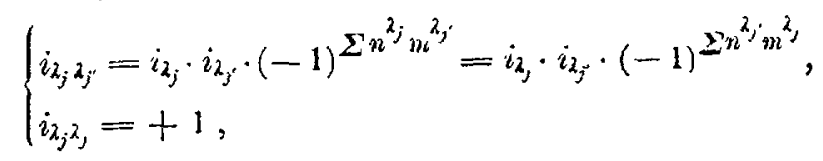

für beliebige $j$ und $j$.

Daher wird nach $(3)$ und $\left(5^{\prime \prime}\right)$ :

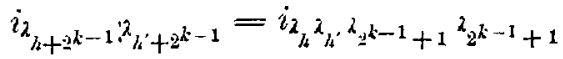

$$
\begin{aligned}
& =i_{\lambda_{k} k_{k}} \cdot i_{2^{k-1}+1} \lambda_{2^{k-1}+1} \cdot(-1)^{\sum n^{\lambda_{k} \lambda_{k^{\prime}}} m^{2 \lambda_{2} k-1}+1}
\end{aligned}
$$

d. h.

(6) $\quad i_{\lambda_{h+2^{k-1}} \lambda_{h^{\prime}+2^{k-1}}}=i_{\lambda_{h} \lambda_{h^{\prime}}}$,

wein $h$ und $h^{\prime}$ wie in (3) gehen.

Wegen dieser Beziehungen (4) und (6) ist es angezeigt, die Gleichung (6) mit

$$
i_{\lambda_{j}}
$$

auf beiden Seiten -zu multipliciren, und sodann statt der Grössen $c_{e}^{(j)}$ die folgenden einzuführen:

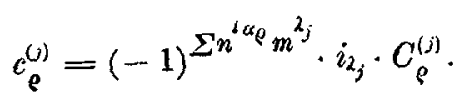

Das Additionstheorem nimmt dann an Stelle von (1), (2) vermöge (5) die Form an:

$$
\begin{gathered}
\vartheta_{0}(u+v+w) \vartheta_{0}(u-v) \\
=\sum_{\rho} \sum_{j} C_{\rho}^{(\jmath \jmath)} \cdot(-1)^{\sum n^{b u_{\rho}} m^{j_{j}} \cdot i_{\lambda_{j}} \cdot v_{\lambda_{j} u_{\rho}}(u) \cdot \vartheta_{\lambda_{j} u_{\rho}}(u+w),} \\
\left(\begin{array}{l}
\rho=0,1, \ldots, 7, \\
j=1,2,3, \ldots, 2^{p-3}
\end{array}\right)
\end{gathered}
$$

wo die $C_{\varrho}^{(j)}$ bestimmt sind durch

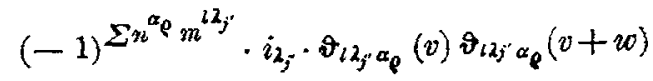

$$
\begin{aligned}
& =\sum_{j} C_{e}^{(j)} i_{i_{j} \lambda_{j}} \hat{v}_{i \lambda_{j} \lambda_{j}} \cdot \vartheta_{l \lambda_{j} \lambda_{j}}(w), \\
& \left(j^{\prime \prime}=1,2,3, \cdots, 2^{p-3}\right), \\
& =\sum_{j} \delta_{j, j} c_{e}^{(n)}
\end{aligned}
$$


wenn man hier noch

$$
i_{\lambda_{j} \lambda_{j}} \boldsymbol{v}_{l \lambda_{j} \lambda_{j}} \cdot \vartheta_{l \lambda_{j} \lambda_{j^{\prime}}}(w)=\delta_{j_{1} j^{\prime}}=\delta_{j_{, j},}
$$

setzt, wobei

$$
\delta_{4+2^{k-1}, h^{+}+2^{k-1}}=\delta_{h, h^{*}}
$$

und die $h, h$, wie in (3) genommen werden.

\section{$\S 15$.}

Eine besondere Classe von Determinanten.

Die Determinante der Coefficienten der $2^{\mu}$ Gleichungen (II), § 14 . (für festes $\varrho$ und $\mu=p-3$ ) ist

Wo

$$
\Delta=\left|\begin{array}{cccc}
\delta_{1,1} & \delta_{1,2} & \cdots & \delta_{1,2^{\mu}} \\
\delta_{2,1} & \delta_{2,2} & \cdots & \delta_{2,2^{\mu}} \\
\cdot & \cdot & \cdots & \cdot \\
\delta_{2^{\mu}, 1} & \delta_{2^{\mu}, 2} & \cdots & \delta_{2^{\mu}, 2^{\mu}}
\end{array}\right|
$$

für

$$
\delta_{h^{2} 2^{k-1}, h^{\prime}+2^{k-1}}=\delta_{h, h^{\prime}}=\delta_{h^{\prime}, h} \text {, }
$$

$$
h, h^{\prime}=j \cdot 2^{k}+1, j \cdot 2^{k}+2, \cdots, j \cdot 2^{k}+2^{k-1},
$$

wo $j=0,1,2, \cdots$ zu setzen ist und $k$ beliebig $=1,2,3 \ldots$ ist, wenn man für die Indices die mod. $2^{\mu}$ congruenten nimmt. Man kann diese Eigenschaft*) der Elemente von $\Delta$ so aussprechen:

Theilt man $\Delta$ in 4 Quadrate von je $2^{\mu-1}$ Reihen, so sind je die beiden quer gegenüberstehenden Theile gleich zusammengesetzt. Jeder dieser 4 Theile hat, bei Theilung in 4 weitere Quadrate, dieselbe Eigenschaft; und so fort bei fortgesetzter Theilung in je 4 Quadrate von gleich viel Reihen.

Setzt man also

$$
\delta_{1, j}=\delta_{j}, \quad\left(j=1,2, \cdots, 2^{\mu}\right),
$$

So kann man $\Delta$ so schreiben:

*) Der Fall $\mu=2$ findet sich an mehreren Orten, der allgemeine Fall in einer (mir erst zur Zeit der Correctur bekannt gewordenen) Note von Puchta im XXXVПI. Bd. der Denkschriften d. Wiener Akademie (1878), p. 315. Debrigens ist aach dieser Fall in einer Determinante von viel allgemeinerer Form, aber analogen Eigenschaften enthalten, wie ich in einer demnächst erscheinenden Notiz zeigen werde. 


$$
\begin{array}{cccccccccc}
\delta_{1} & \delta_{2} & \delta_{3} & \delta_{4} & \delta_{5} & \delta_{6} & \delta_{7} & \delta_{3} & \\
\delta_{2} & \delta_{1} & \delta_{4} & \delta_{3} & \delta_{6} & \delta_{5} & \delta_{3} & \delta_{7}
\end{array} .
$$

Vermöge dieser Eigenschaft lässt sich $\Delta$ darstellen als das Product vo $2^{\mu}$ Factoren, die alle linear sind in den Elementen von $\Delta$.

Um dies $\mathrm{zu}$ zeigen, multiplicire man die $2^{1 \mathrm{c}}, 4^{\text {te }}, 6^{\mathrm{te}}, 8^{1 \mathrm{c}}, \ldots$ Horizontalreibe, ebenso dieselben Verticalreihen je mit $\varepsilon_{1}$; sodann die $3^{\text {te }}$ and $4^{\text {te }}, 7^{\text {te }}$ und $8^{\text {te }}, 11^{\text {te }}$ und $12^{\text {te }}, \ldots$ Horizontal- and Verticalreihe je mit $\varepsilon_{2}$; etc., allgemein also multiplicire man die $(h+2 h-1)^{\text {le }}$ Horizontal- und Verticalreihe, wo $h$ die Werthe

$$
\begin{gathered}
j \cdot 2^{k}+1 . j \cdot 2^{k}+2, \cdots, j \cdot 2^{k}+2^{k-1} \\
\quad\left(\text { für } j=0,1,2, \cdots, 2^{\mu-k}-1\right)
\end{gathered}
$$

anzunehmen hat, je mit $\varepsilon_{k}$, von $k=1,2, \cdots, \mu$. Dabei sei

$$
\varepsilon_{1}^{2}=\varepsilon_{2}^{2}=\cdots=\varepsilon_{\mu}^{2}=1 \text {. }
$$

Hierdurch wird zunächst der Werth der Determinante $\Delta$ nicht geändert.

Weiter bleiben zwei Elemente von $\Delta$, die vorher einander gleich waren, auch nach der Multiplication mit den Grössen $\varepsilon_{k}$ einander gleich. Denkt man sich nämlich $\Delta$ in 4 Theile I, II, III, IV von je $2^{\mu-1}$ Reihen getheilt, wo $\mathrm{I}=\mathrm{IV}, \mathrm{II}=\mathrm{III}$, so bewirkt die Multiplication mit $\varepsilon_{\mu}$, dass alle Elemente von I unverändert bleiben, die von IV mit $\varepsilon_{\mu}{ }^{2}=1$, die von II und III je mit $\varepsilon_{\mu^{\prime}}$ multiplicirt werden. Die Multiplication mit $\varepsilon_{\mu-1}$ bewirkt, dass von den 4 entsprechenden Theilen $\mathrm{I}^{\prime}, \mathrm{II}^{\prime}, \mathrm{III}^{\prime}, \mathrm{IV}^{\prime}$ von I die Elemente der gegenüberstehenden Theile $I^{\prime}, I^{\prime}$ mit 1 , bez. $\varepsilon_{\mu-1}^{2}=1$, die Elemente von II' und III' je mit $\varepsilon_{\mu-1}$ multiplicirt werden; und analog die 4 Theile von II, von III und von IV. Ebenso sieht man, indem man jeden dieser Theile $\mathrm{I}^{\prime}, \mathrm{Il}^{\prime}$ etc. wieder in je 4 Theile zerlegt, dass die Multiplication mit $\varepsilon_{\mu-2}$ vorher gleiche Elemente auch gleich lässt; etc.

Die 'Determinante $\Delta$ wird also nach der Multiplication mit den Grössen $\varepsilon_{1}, \varepsilon_{2}, \cdots, \varepsilon_{\mu-1}$ genau von derselben Art, wie sie ursprünglich definirt war. Addirt man nun sämmtliche Verticalreihen, von der 
zweiten an, zur ersten Verticalreihe, so werden sämmtliche Elemente dieser Reihe einander gleich, und zwar gleich:

$$
\begin{aligned}
& \left(\delta_{1}+\varepsilon_{1} \delta_{2}\right)+\varepsilon_{2}\left(\delta_{3}+\varepsilon_{1} \delta_{4}\right)+\varepsilon_{3}\left[\left(\delta_{5}+\varepsilon_{1} \delta_{6}\right)+\varepsilon_{2}\left(\delta_{7}+\varepsilon_{1} \delta_{s}\right)\right]+\cdots \\
= & \delta_{1}+\varepsilon_{1} \delta_{2}+\varepsilon_{2} \delta_{3}+\varepsilon_{1} \varepsilon_{2} \delta_{4}+\varepsilon_{3} \delta_{3}+\varepsilon_{1} \varepsilon_{3} \delta_{6}+\varepsilon_{2} \varepsilon_{3} \delta_{7}+\varepsilon_{1} \varepsilon_{2} \varepsilon_{3} \delta_{5}+\varepsilon_{4} \delta_{9}+\cdots,
\end{aligned}
$$

wo die Combinationen der $\varepsilon_{k}$ genau so geordnet sind, wie die Grössen $\lambda_{j}$ in $\$ 14$. Dieser Ausdruck geht also als Factor aus $\Delta$ heraus. Und da die $\varepsilon_{k}$ hier beliebig die Werthe +1 oder -1 annehmen konnten, so hat man für die Determinante $\Delta$ die Darstellung:

$$
\begin{gathered}
\Delta=\Pi_{\varepsilon}\left\{\delta_{1}+\varepsilon_{1} \delta_{2}+\varepsilon_{2} \delta_{3}+\varepsilon_{1} \varepsilon_{2} \delta_{1}+\varepsilon_{3} \delta_{i}+\varepsilon_{1} \varepsilon_{3} \delta_{i}+\varepsilon_{2} \varepsilon_{3} \delta_{i}\right. \\
\left.+\varepsilon_{1} \varepsilon_{2} \varepsilon_{3} \delta_{s}+\cdots+\varepsilon_{1} \varepsilon_{2} \cdots \varepsilon_{14} \delta_{2^{4}}\right\} \\
\text { für } \varepsilon_{k^{2}}{ }^{2}=1, \quad(k=1,2, \cdots, \mu)
\end{gathered}
$$

wo das Product $\Pi_{\varepsilon}$ auf alle $2^{\mu}$ Werthsysteme, welche die Grössen $\varepsilon_{k}$ annehmen können, auszudehnen ist.

Die ersten Unterdeterminanten von $\Delta$, nach irgend zwei einander gleichen Elementen genommen, sind ebenfalls einander gleich.

Denn zunächst sind wieder, bei Zerlegung von $\Delta$ in die vier Theile I, II, III, IV, die Unterdeterminanten nach den Elementen von I gleich denen nach den entsprechenden Elenenten von IV, die nach den Elementen von II gleich deuen nach den entsprechenden Elomenten von III, da durch Vertauschuug der $1^{\text {ten }}, 2^{\text {ten }}, \cdots\left(2^{\mu-1}\right)^{\text {ten }}$ Horizontalund Verticalreihe bez. mit der $\left(2^{\mu-1}+1\right)^{\text {ten }},\left(2^{\mu-1}+2\right)^{\text {ten }}, \cdots,\left(2^{\mu}\right)^{\text {leo }}$ Reihe nur I in IV, II in III übergeht, also die Elemente von $\Delta$ unverändert bleiben. Ebenso sind, bei Zerlegung von $I$ in die vier Theile $\mathrm{I}^{\prime}, \mathrm{II}^{\prime}, \mathrm{III}^{\prime}, \mathrm{IV}^{\prime}$ die Unterdeterminanten nach den Elementen von $\mathrm{I}^{\prime}$ gleich denen nach den entsprechenden Elementen ron IV', etc.

Diese Unterdeterminanten bilden also ein System von derselben Art, wie die Elemente $\delta_{j, 5}$ von $\Delta$ sellust. Bezeichnet man insbesondere die Unterdetermivanten, genommen nach den Elementen

von $\Delta$, entsprechend mit

$$
\delta_{1}, \delta_{2}, \cdots, \delta_{2}^{\mu}
$$

$$
\Delta_{1}, \Delta_{12}, \cdots, \Delta_{2 \mu}
$$

so wird die Determinante, gebildet aus diesen erșten Unterdeterminanten: $=\Delta^{3^{\mu}-1}=\Pi \varepsilon\left\{\Delta_{1}+\varepsilon_{1} \Delta_{2}+\varepsilon_{2} \Delta_{3}+\varepsilon_{1} \varepsilon_{2} \Delta_{4}+\varepsilon_{3} \Delta_{5}+\cdots+\varepsilon_{1} \varepsilon_{2} \cdots \varepsilon_{\mu^{\mu}} \Delta_{2^{\mu}}\right\} ;$ und ferner folgt, indem man die zweite, dritte, vierte, . . Verticalreihe von $\Delta$ der Reihe nach mit $\varepsilon_{1}, \varepsilon_{2}, \varepsilon_{1} \varepsilon_{2}, \ldots$ multiplicirt, dieselben zur ersten Verticalreihe addirt und dann nach den Elementen dieser Reihe ordnet:

$$
\begin{aligned}
\Delta= & \left\{\delta_{1}+\varepsilon_{1} \delta_{2}+\varepsilon_{2} \delta_{3}+\varepsilon_{1} \varepsilon_{2} \delta_{4}+\varepsilon_{3} \delta_{3}+\cdots+\varepsilon_{1} \varepsilon_{2} \cdots \varepsilon_{\mu} \cdot \delta_{2 \mu}\right\} \\
& \cdot\left\{\Delta_{1}+\varepsilon_{1} \Delta_{2}+\varepsilon_{2} \Delta_{3}+\varepsilon_{1} \varepsilon_{2} \Delta_{4}+\varepsilon_{3} \Delta_{5}+\cdots+\varepsilon_{1} \varepsilon_{2} \cdots \varepsilon_{\mu} \cdot \Delta_{2}\right\}
\end{aligned}
$$


wo die $\varepsilon_{1}, \varepsilon_{2}, \cdots, \varepsilon_{\mu}$ beliebig die Werthe +1 oder -1 annehmen können.

$\S 16$.

Das Additionstheorem in expliciter Gestalt. Fundamentalformel.

Um $\S 15$. auf die Formel (I), (II) des $\S 14$. anzuwenden, hat man dort $\mu=p-3$ zu setzen und

$$
\delta_{j}=\delta_{1, j}=i_{i_{j}} \boldsymbol{\vartheta}_{i \lambda_{j}} \cdot \vartheta_{l \lambda_{j}}(w) .
$$

Danu wird die Determinante der Gleichungen II, \& 14.:

$$
\begin{aligned}
& \Delta=\Pi_{\varepsilon}\left\{\delta_{1}+\varepsilon_{1} \delta_{2}+\varepsilon_{2} \delta_{3}+\varepsilon_{1} \varepsilon_{2} \delta_{1}+\varepsilon_{3} \delta_{5}+\cdots+\varepsilon_{1} \varepsilon_{2} \cdots \varepsilon_{p-3} \cdot \delta_{2 p-3}\right\}
\end{aligned}
$$

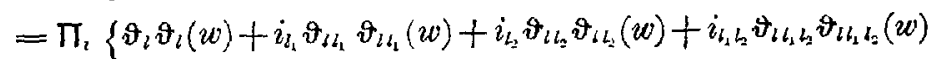

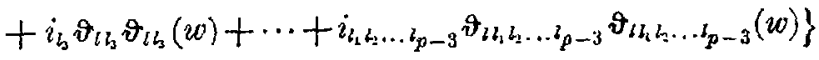

$$
\begin{aligned}
& =\Pi_{i}\left[\sum_{j} i_{\lambda_{j}} \vartheta_{l \lambda_{j}} \cdot \vartheta_{l \lambda,}(w)\right]
\end{aligned}
$$

wo das erste Product $\Pi_{\varepsilon}$, bei fest angenommenen Grössen $i_{\lambda_{j}}$ auszudehnen ist über alle $2^{p-3}$ Werthsysteme, welche die Grössen $\varepsilon_{k}$ annehnen können, wenn

$$
\varepsilon_{1}^{2}=\varepsilon_{2}^{2}=\cdots=\varepsilon_{p-3}^{2}=1 ;
$$

und wo das zweite Product $\Pi_{i}$ auszudehnen ist über alle $2^{p-3}$ Wertlsysteme, welche die Grössen $i_{\lambda_{j}}$ nach $(5),\left(5^{\prime}\right)$ des $\$ 14$. annehmen können, wenn die

gegeben sind.

$$
\left(i_{l_{k}}\right)^{2}=(-1)^{\sum n^{i_{k}} m^{i_{k}}}
$$

Bei allgemein gelassenen Moduln der Thetafunctionen kann diese Determinante $\Delta$ nicht verschwinden. Denn dann existirte eine für alle $w$ identische lineare Relation zwischen den $2^{p-3}$ Grössen

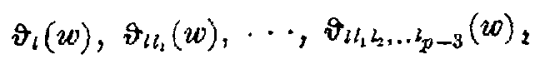

was nach der Definition der $\vartheta$-Functionen allgemein nicht der Fall ist.

Bezeichnet man weiter die Unterdeterminanten von $\Delta$, genommen nach dem Element $\delta_{j, j^{\prime}}$, mit $\Delta_{j, 5^{\prime}}$, wo also:

wenn

$$
\Delta_{l, k^{\prime}}=\Delta_{j, j^{\prime}}
$$

$$
\delta_{h, i^{\prime}}=d_{s, j^{\prime}} \text {, }
$$

so ergiebt die Auflösung von (1), (II), § 14,, das Additionstheorem in der Form: 


$$
\Delta \cdot \vartheta_{0}(u+v+v) \cdot \vartheta_{0}(u-v)
$$

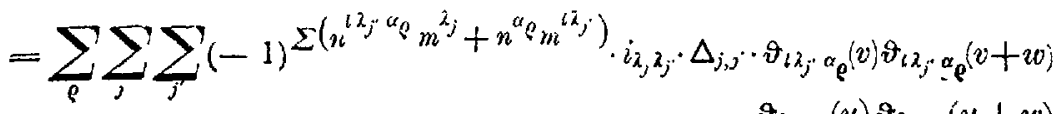

$$
\begin{aligned}
& \left(\begin{array}{c}
\rho=0,1, \cdots, 7 \\
j, j=1,2,3, \cdots, 2^{p-3}
\end{array}\right) . \\
& \cdot \boldsymbol{v}_{\lambda_{j} \alpha_{\varrho}}(u) v_{\lambda_{j} \alpha_{\rho}}(u+w) \text {. }
\end{aligned}
$$

In dieser Form des Additionstheorems besteht jeder Coefficient eines Gliedes

$$
\vartheta_{\lambda_{j} \alpha_{\ell}}(u) \vartheta_{\lambda_{j} c_{e}}(u+w)
$$

noch aus einer Summe von $2^{p-3}$ Glitedern der Form

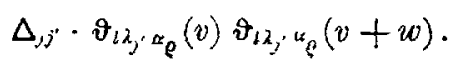

Wir wollen jetzt zeigen, dass unter Benutzung der Eigenschaften von $\Delta$ eine neve Formel herzustellen ist, bei welcher die Coefficienten der rechten Seite alle cingliedrig werden, und welche als Fundamentalformel der Theorie zu betrachten ist.

Zunächst setzen wir in (III):

$$
u=v^{\prime}+\frac{1}{2} \varpi^{i \lambda / 4}, \quad\left(h=1,2, \cdots, 2^{p-5}\right)
$$

wodurch (III) übergeht in:

$$
\begin{aligned}
& \Delta \cdot \vartheta_{l \lambda_{h}}\left(v+v^{\prime}+w\right) \cdot \vartheta_{i \lambda_{h}}\left(v^{\prime}-v\right)
\end{aligned}
$$

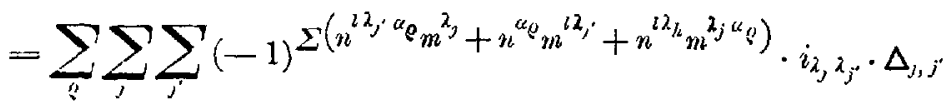

$$
\begin{aligned}
& \text { - } \vartheta_{i \lambda_{j}, u_{e}}(v) \vartheta_{l \lambda_{j}, u_{Q}}(v+w) \cdot \vartheta_{l \lambda_{l} \lambda_{j} \alpha_{Q}}\left(v^{\prime}\right) \vartheta_{l \lambda_{h} \lambda_{j} \alpha_{e}}\left(v^{\prime}+w\right) .
\end{aligned}
$$

Man multiplicire un diese Gleichung auf beiden Seiten mit

$$
(-1)^{\Sigma n^{\lambda_{h} \alpha_{0}} m^{\lambda_{h} \alpha_{0}}} \cdot \vartheta_{l \lambda_{h}} \vartheta_{l \lambda_{h}}(w)=(-1)^{\Sigma\left(n^{\lambda_{h} \alpha_{0}} m^{\lambda_{h} \alpha_{0}}+n^{\lambda_{h}} m^{\lambda_{h}}\right)} \cdot i_{\lambda_{h}} \cdot \delta_{h},
$$

und addire alsdann über alle Werthe von $h=1,2, \ldots ; 2^{p-3}$. Die alsdann auf der rechten Seite vorzunehmenden Summationen sollen so ausgeführt werden: zunächst lasse man $\varrho$ und $j^{\prime}$ fest, nebme denjenigen Werth von $j$, für welchen

$$
\lambda_{j} \lambda_{h}=\lambda_{h i} L^{z}
$$

wo $L$ ein Product der $l_{k}$, also auch $\left(\left(5^{\prime \prime}\right), \S 14.\right)$ :

und ferner:

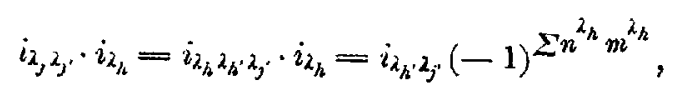

$$
\Delta_{j, j^{\prime}}=\Delta_{\lambda_{h^{2} h^{\prime}, \lambda^{\prime}}}=\Delta_{\lambda_{k}, \lambda_{h^{\prime} \lambda_{j}}}=\Delta_{h, h^{\prime} j^{\prime}},
$$


und summire über alle Werthe von $h$. Das hiervou herrühreude Glied, unter den Summenzeichen $\sum_{\rho} \sum_{j} \sum_{k^{\prime}}$, wird

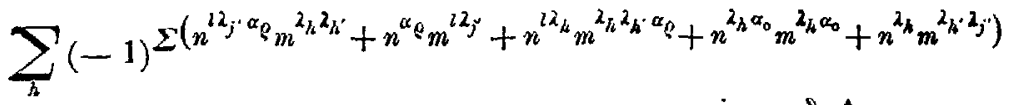

$$
\begin{aligned}
& \cdot i_{\lambda_{h}, z_{j}} \cdot \delta_{h} \Delta_{h, i} ; j
\end{aligned}
$$

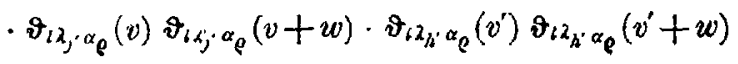

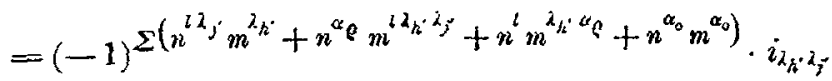

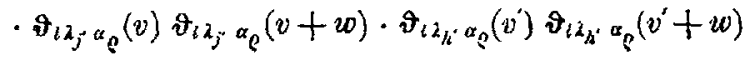

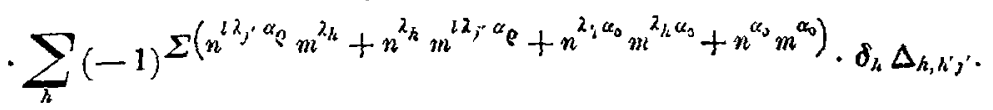

Der Exponent von -1 im letzten Factor $\sum_{h}$ wird aber $=$

$$
\begin{aligned}
& =\sum\left(n^{\lambda^{\prime}} m^{\lambda_{h}}+n^{\lambda_{h}} m^{{ }^{j_{j}}}\right)+\sum n^{2 \lambda_{h} \alpha_{\nu} \alpha_{Q}} m^{j \lambda_{l} \alpha_{\nu} \alpha_{Q}}+\sum n^{i \alpha_{0} \alpha_{\rho}} m^{l \alpha_{0} \alpha_{\varphi}} \\
& \equiv 0+1+1 \equiv 0(\bmod .2)
\end{aligned}
$$

also wird der ganze Factor $\sum_{i}$ zu

$$
\sum_{h} \delta_{h} \Delta_{h, l^{\prime} j^{\prime}}=\Delta \text { oder }=0
$$

je nachdem $h^{\prime}=j^{\prime}$ oder $h^{\prime} \gtrless j^{\prime}$.

Daher bleiben auf der rechten Seite nur diejenigen Glieder übrig, für welche $h^{\prime}=j^{\prime}$, d. h.

$$
\lambda_{j} \lambda_{l}=\lambda_{j} L^{2}
$$

Glieder, die alle $\Delta$ zum Factor haben. Indem man diesen auf beiden Seiten weghebt (und noch $j^{\prime}$ mit $j$ vertauscht), folgt:

$$
\begin{aligned}
& \text { (V) } \sum_{k=1}^{b=2^{p-3}}(-1)^{\Sigma\left(n^{\lambda_{h}} m^{\lambda_{k}}+n^{\lambda_{k}} m^{\alpha_{n}}+n^{\alpha_{j}} m^{z_{h}}\right)} \cdot \vartheta_{l \lambda_{k}} \boldsymbol{v}_{l \lambda_{h}}(w) \\
& \text { - } \vartheta_{b \lambda_{h}}\left(v+v^{\prime}+w\right) \vartheta_{b i_{h}}\left(v^{\prime}-v\right)
\end{aligned}
$$

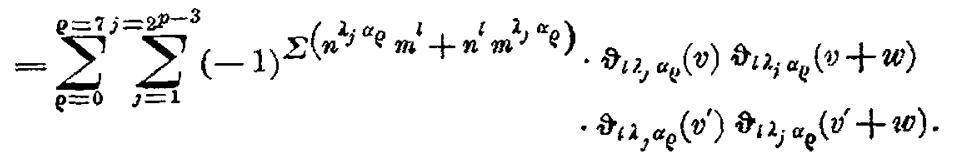

Dieses ist die Fundamentalformel der ganzen Theorie, für $p \geqq 3$. Bei ihr ist die linke Seite eine Summe von $2^{p-3}$ Grössen mit zusammengesetzten Argumenten, die rechte Seite eine Summe von $2^{p}$ Grössen, 
deren Coefficienten eingliedrig geworden sind. Setzt man $v^{\prime}+\frac{1}{2} \omega^{2^{\prime}}$ an Stelle von $v^{\prime}$, und giebt $j^{\prime}$ alle Werthe von $1,2, \ldots, 2^{p-3}$, so erhält man $2^{\mu-3}$ Gleichungen, aus welchen durch Auflösung wieder (III) oder (IV) hervorgeht.

\section{$\S 17$.}

\section{Speciellere Formeln.}

Die bisher gegebenen allgemeinen Theoreme sind in einer Form mitgetheilt, in welcher sie für alle $p \geqq 3$ gelten. Wir geben jetzt, durch besondere Annahmen für die Grössen $w, v, u$, eine Reibe speciellerer Relationen, von denen die ersten noch für alle $p \geqq 3$, die rächsten nur für $p \geq 4$, die dritten für $p \geq 5$ und die letzten nur für $p \geqq 6$ Geltung haben.

1) $p \geq 3$.

Nach $\S 13$. liefern die Ausdrücke $\left(l \lambda_{j}\right)$, verbundea wit 2 oder 6 der Grössen

$$
\left(\alpha_{0}\right),\left(\alpha_{1}\right), \cdots,\left(\alpha_{7}\right)
$$

ungerade, mit keiner oder 4 derselben verbunden gerade Charakteristiken. Daher verschwindet nach \& 12. die Grösse

$$
v_{l \lambda_{j} a_{e}}(v)
$$

wenn $v=\frac{1}{2} \varpi^{p}$, und $(\beta)=\left(\alpha_{\sigma}\right)$, wo $\sigma \gtrless \varrho$, oder $(\beta)$ aus dreien der $\left(\alpha_{c}\right)$, von denen eines $=\left(\alpha_{0}\right)$ ist, zusammengesetzt ist. Man setze also $\left.{ }^{*}\right)$ :

$$
v=\frac{1}{2} \widetilde{\omega}^{\alpha_{1} \alpha_{i} \alpha_{s}}, v^{\prime}=u+\frac{1}{2} \widetilde{\omega}^{\alpha_{3} \alpha_{s} \alpha_{r}},
$$

so geht $(V)$ nach einigen aus $\& 12$. folgenden Reductionen über in:

$$
\begin{aligned}
& \text { (VI) } 0=\sum_{h=1}^{k=2^{\nu-3}}(-1)^{\sum n^{\lambda_{h}} m^{\lambda_{h}}} \cdot \vartheta_{l \lambda_{h}} \vartheta_{l \lambda_{h}}(w) \cdot \vartheta_{l \lambda_{k}}(u) \vartheta_{l \lambda_{h}}(u+w)
\end{aligned}
$$

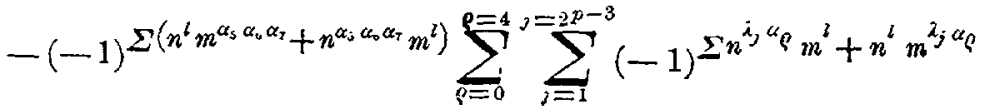

$$
\begin{aligned}
& \text { - } \vartheta_{l \lambda_{j} \alpha_{\rho} \alpha_{s} \alpha_{s} \alpha_{z}} \vartheta_{l \lambda_{j} \alpha_{\rho} \alpha_{s} \alpha_{s} \alpha_{7}}(w) \cdot \vartheta_{l \lambda_{j} \alpha_{g} \alpha_{s} \alpha_{0} \alpha_{7}}(u) \vartheta_{l \lambda_{j} \alpha_{\rho} \alpha_{s} \alpha_{t} \alpha_{z}}(u+w) \text {, }
\end{aligned}
$$

was eine Beziehung zwischen 6.2p-3 Producten der Form $\vartheta_{a}(u) \vartheta_{t}(u+w)$ mit Coefficienten der Form $\vartheta_{\varepsilon} \vartheta_{\varepsilon}(w)$ vorstellt.

*) Nach den Definitionen des $\$ 12$. wäre eigentlich zu schreiben $\vartheta_{0 ! \lambda_{j} \alpha_{e}}(v)$ an Stelle roa $\vartheta_{i \alpha_{j} \alpha_{e}}(v)$, und $\frac{1}{2} \omega^{0 \alpha_{s} \alpha_{0} \alpha_{s}}$ statt $\frac{1}{2} \omega^{\alpha_{5} \alpha_{0} \alpha_{7}}$, da in $\frac{1}{2} \omega^{\beta}$ das $\beta$ eine Gruppencharakteristik vorstellt. Indessen ist hier und im Folgenden die einfachere Schreibart gewäblt. 
Setzt man ferner in (V):

$$
w=\frac{1}{2} \widetilde{\omega}^{2} \alpha_{0} \alpha_{7},
$$

so verschwindet die ganze linke Seite, und es bleibt:

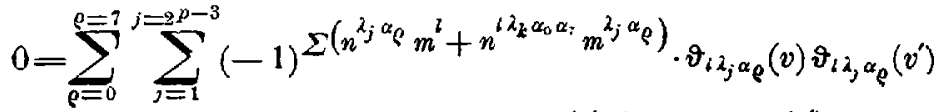

$$
\begin{aligned}
& \cdot \vartheta_{l 2, \lambda_{k} \alpha_{q} \alpha_{0} \alpha_{z}}(v) \vartheta_{l i, \alpha_{k} \alpha_{q} \alpha_{j} \alpha_{z}}\left(v^{\prime}\right),
\end{aligned}
$$

ein aus $2^{p}$ Producten bestehender Ausdruck.

Ferner sei zu gleicher Zeit gesetzt:

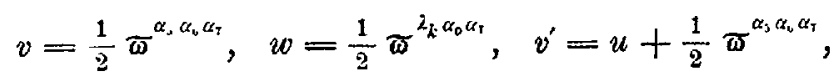

wo $k$ beliebig einen der Werthe $1,2, \cdots, 2^{p-3}$ annehmen kann, so folgt:

$$
\begin{aligned}
& \text { (VIII) } \quad 0=\sum_{\rho=1}^{n=4} \sum_{j=1}^{j=2^{p-3}}(-1)^{\Sigma\left(n^{\lambda_{j} \alpha_{e}} m^{l}+n^{l \lambda_{k} \alpha_{0} \alpha_{i}} m^{\left.\lambda_{j} \alpha_{\rho}\right)}\right.}
\end{aligned}
$$

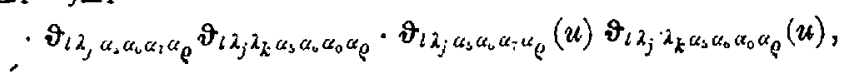

also eine Beziehung zwischen $2^{p-1}$ Producten der Form $\vartheta_{\boldsymbol{z}}(u) \vartheta_{\vartheta^{\prime}}(u)$.

Um eine Formel zu erhalten, welche nur die Quadrate $\vartheta_{9}{ }^{2}(u)$ entbält, setze man in (VI) $w=0$; und bat dann eine Beziehung zwischen $6 \cdot 2^{p-3}$ solchen Quadraten. Setzt man hierin weiter $u=0$, so folgt eine Beziehung zwiscken $6 \cdot 2^{p-3}$ vierten Potenzen von $\vartheta$-Functionen für die 0-Werthe der Argumente:

$$
\begin{aligned}
& 0=\sum_{h=1}^{h=2^{p-3}}(-1)^{\Sigma n^{\lambda_{h}} m^{\lambda_{h}}} \cdot \vartheta_{l \lambda_{h}}^{4}-(-1)^{\Sigma\left(n^{l} m^{\alpha_{s} \alpha_{0} \alpha_{i}}+n^{\alpha_{3} \alpha_{v} \alpha_{7}} m^{l}\right)}
\end{aligned}
$$

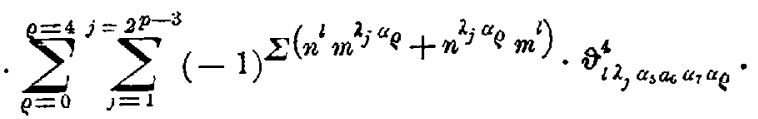

Endlich ergiebt sich eine weitere Beziehung für die 0-Werthe der Argumente, volche aus nur $3 \cdot 2^{p-3}$ Producten besteht, indem man in (VIII) setzt:

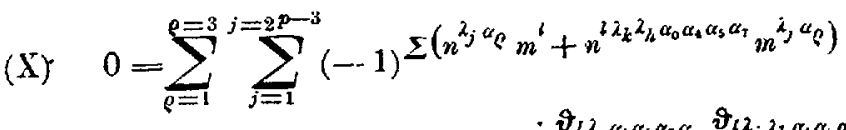

$$
\begin{aligned}
& \text { - } \boldsymbol{\vartheta}_{l \lambda_{j} \alpha_{s} \alpha_{s} \alpha_{7} \alpha_{l}} \mathcal{V}_{l \lambda_{j} \lambda_{k} \alpha_{s} \alpha_{s} \alpha_{0} \alpha_{e}} \\
& \text { - } \vartheta_{t \lambda_{j} \lambda_{k} \alpha_{4} \alpha_{b} \alpha_{z} \alpha_{\ell}} \vartheta_{t \lambda_{j} \lambda_{k} \lambda_{L} \alpha_{4} \alpha_{4} \alpha_{0} \alpha_{\ell}} \text {, }
\end{aligned}
$$


wo die Indices $k$ und $h$ beliebig die Werthe $1,2, \cdots, 2^{p-3}$ annehmen dürfen.

Von den Specialisirungen der Formel (III), $\$ 16$. ist hier nur diejenige zu bemerken, welche für

$$
v=\frac{1}{2} \widetilde{w}^{\alpha_{s} \psi_{n} \alpha_{n}}
$$

eintritt. Dann verschwinden einfach auf der rechten Seite alle Glieder für $\varrho=5,6$ und 7 , und man erhält eine auch aus den Gleichungen (VI) folgende Formel.

2) $p \geq 4$.

Die in $\$ 14$, gegebene Zusammensetzung der $\lambda_{k}$ aus den $l_{k}$, und die Bedeutung dieser Grössen $l_{k}$ und $l$ nach $\$ 13$, zeigen, dass für $p>4$ die Grössen

$$
\left(l \lambda_{h}\right),
$$

ausgedrückt durch die Combinatiouen der Charabteristiken

$$
\left(\alpha_{0}\right),\left(\alpha_{1}\right),\left(\alpha_{2}\right), \cdots,\left(\alpha_{2 p}\right),\left(\alpha_{2 p+1}\right),
$$

in diesen Ausdrücken explicite $\left(\alpha_{2 p}\right)$, aber nicht $\left(\alpha_{2 p+1}\right)$ enthalten, wenn $h=1,2, \ldots, 2^{p-4} ;$ dagegen $\left(\alpha_{2 p+1}\right)$ und nicht $\left(\alpha_{2 p}\right)$, wenn $h_{h}=2^{p-4}+1, \cdots, 2^{p-3}$. Daher wird z. B. $\left(l \lambda_{k} \kappa_{1} c_{z p}\right)$ im ersteren Falle gerade, im zweiten Falle ungerade.

Man setze also in Formel (V):

$$
w=\frac{1}{2} \varpi^{\alpha_{i} \alpha_{2} p}
$$

und erhält dann aus (V) einen Ausdruck, in welchem die linke Seite wur über lie Werthe $h=1,2, \ldots, \underline{.}^{p-4}$ zu summiren ist, also einen Ausdruck von $17 \cdot 2^{p-4}$ Gliedern.

Setat man ferner zu gleicher \%eit:

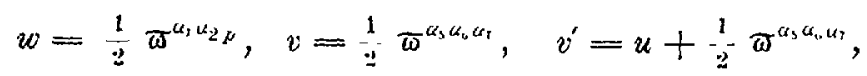

so verschwinden auf der linken Seite von (V) alle Glieder für $h=2^{p-4}+1, \cdots, 2^{p-3}$; auf der rechten Seite alle Glieder für $\varrho=5,6,7$, und ferver diejenigen für $\varrho=0,1, \cdots, 4$, wenn zugleich $j=1,2, \cdots, 2^{p-4}$. Daher geht $(\mathrm{V})$ über in:

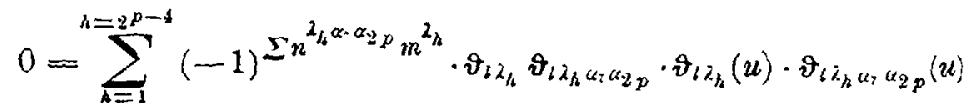

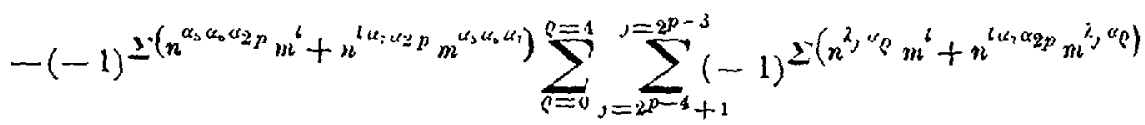

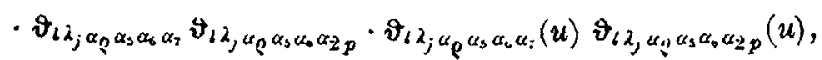


eine Relution zwischen 6. $2^{p-4}$ Producten der Form $\vartheta_{*}(u) \vartheta_{*}(u)$. Dieselbe Art von Relation, in etwas geschlossenerer Gestalt, erbält wan, wenn man in (V) setzt:

$$
w=\frac{1}{2} \pi^{\alpha_{0} \alpha_{2} \alpha_{2 p} \alpha_{2 p+1}}, \quad v=\frac{1}{2} \widetilde{\sigma}^{\alpha_{2 p}},
$$

nämlich:

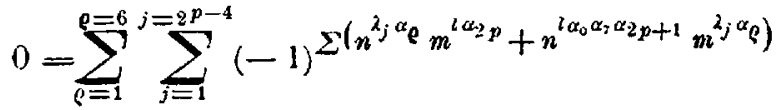

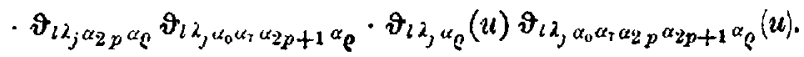

Insbesoudere folgt, indem man in $\left(\mathrm{XI}^{\circ}\right)$

$$
u=\frac{1}{2}{\pi^{2}}^{2_{h} \alpha_{a} \alpha_{s} \alpha_{c}}, \quad\left(\text { wo } h=1,2, \cdots, \text { oder } 2^{y-4}\right)
$$

setzt, eine Relation zwischen $2^{p-2}$ Producten, für die 0.Werthe der Argumente:

$$
\begin{aligned}
& 0=\sum_{\rho=1}^{\rho=4} \sum_{j=1}^{j=2^{p-4}}(-1)^{\Sigma\left(n^{\lambda_{j} \alpha_{\ell}}\right.} m^{l \alpha_{0} \alpha_{2} p}+n^{2 \alpha_{h} \alpha_{s} \alpha_{0} \alpha_{7} \alpha_{2 p+1}} m^{\left.\lambda_{j} \alpha_{e}\right)}
\end{aligned}
$$

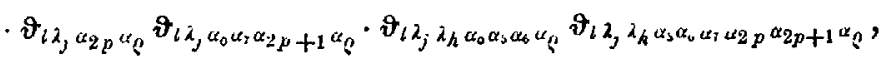

$$
\begin{aligned}
& \text { (wo } h=1,2, \cdots, 2^{p-1} \text { sein darf), }
\end{aligned}
$$

und eine Relation derselben Art, wenn man in (XI)

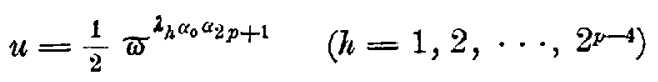

einsetzt.

Setzt man ferner in $(\mathrm{V})$ :

$$
v=\frac{1}{2} \bar{\omega}^{\alpha_{2} p}, \quad v^{\prime}=\frac{1}{2} \omega^{\alpha_{2} p}+u,
$$

so folgt:

$$
\begin{aligned}
& \text { (XIII) } 0=(-1)^{\Sigma\left(n^{l} m^{\alpha_{z p}}+n^{\alpha_{2 p}} m^{l}\right)}
\end{aligned}
$$

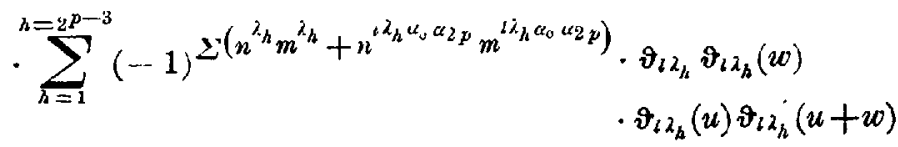

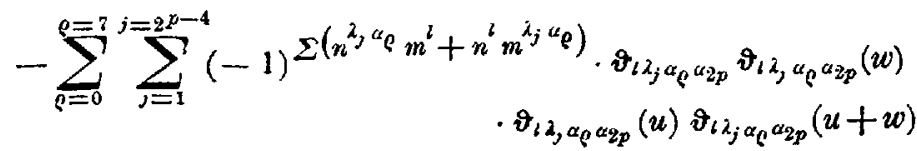

eine Bexiehung zwischen $10 \cdot 2^{p-4}$ Producten der Form $\boldsymbol{\vartheta}_{\bullet}(u) \cdot \vartheta_{\bullet}(u+w)$. Für $w=0$ erhält man hieraus eine Beziehung zwischen $10 \cdot 2^{p-4}$ 
Quadraten $\vartheta_{a}^{2}(u)$, und für $w=0, u=0$ eine Beziehung zwischen 10. $2^{p-4}$ vierten Potenzen $\vartheta_{e}^{4}$ von $\vartheta-F u n c t i o n e n$ für die 0 -Werthe der Argumente.

Von den Specialisirungen der formel (III) ist hier diejenige zu bemerken, welche für

$$
w=\frac{1}{2} \cdot \widetilde{\omega}^{\alpha_{7} \alpha_{2 p}}
$$

eintritt. Die Determinante $\Delta$ wird alsdann das Quadrat der Determinante $\Delta^{\prime}$, welche sich, analog wie $\Delta$ aus den Grössen $\delta_{1}, \delta_{2}, \cdots, \delta_{2}^{p-3}$, so nur aus den Grössen $\delta_{1}, \delta_{2}, \cdot \cdot, \delta_{2} p-4$ zusammensetzt. An Stelle von $\Delta_{j, j}$, tritt $\Delta^{\prime} \cdot \Delta_{j, j}^{\prime}$. Auf der rechten Seite von (III) sind die Summen nur über die Werthe $1,2,3, \ldots, 2^{p-4}$ von $j^{\prime}$ auszudehnen, wenn $j$ die Werthe $1,2, \cdots, 2^{p-4}$ hat; und über $j^{\prime}=2^{p-4}+1, \cdots, 2^{p-3}$, wenn auch $j$ einen dieser Werthe hat. Jedes Glied

$$
\vartheta_{\lambda_{j} \alpha_{\varphi}}(u) \quad \vartheta_{\lambda_{j} \alpha_{q} \alpha_{2 p} \alpha_{Q}}(u)
$$

hat also dann eiven Coefficienten, der aus einer Summe von nur $2^{p-4}$ Gliedern besteht. - Setzt man ausserdem noch

$$
v=\frac{1}{2} \widetilde{\omega}^{\alpha_{5} \alpha_{v} \alpha_{2}},
$$

so bleiben auf der rechten Seite nur noch die Summationen für $\rho=0,1, \cdots, 4 ; j=2^{p-4}+1, \cdots, 2^{p-3} ; j^{\prime}=2^{p-4}+1, \cdots, 2^{p-3}$ auszuführen.

3) $p \geqq 5$.

Die Grössen $\left(l \lambda_{k}\right)$, ausgedrückt durch die Combinationen der Charakteristiken

$$
\left(\alpha_{0}\right),\left(\alpha_{1}\right), \cdots,\left(\alpha_{2 p+1}\right),
$$

sind bez. von den Formen

$$
\left(L \alpha_{2 p-2} \alpha_{2 p}\right) ;\left(L \alpha_{2 p-1} \alpha_{z_{p}}\right) ;\left(L \alpha_{2 p-2} \alpha_{2 p+1}\right) ;\left(L \alpha_{2 p-1} \alpha_{2 p+1}\right),
$$

je nachdem $\lambda_{l}$ bez. von den Formen:

$$
\begin{gathered}
1,2, \cdots, 2^{p-5} ; 2^{p-5}+1, \cdots, 2^{p-4} ; 2^{p-4}+1, \cdots, 2^{p-4}+2^{p-5} \\
2^{p-4}+2^{p-5}+1, \cdots, 2^{p-3}
\end{gathered}
$$

wobei $L$ aus den $\alpha_{j}, \alpha_{1}, \cdots, \alpha_{2 p-3}$ und (für ungerades $p$ ) aus $\alpha_{0} z u-$ sammengesetzt ist. Daher ist $\left(l \lambda_{k} \alpha_{2 p-2} \alpha_{2 p+1}\right)$ im ersten und vierten Falle gerade, im zweiten und dritten Falle ungerade.

Setat man also in $(V)$ :

$$
w=\frac{1}{2} \varpi^{\alpha_{2} p-2}{ }^{\alpha_{2 p+1}}, \quad v==-\frac{1}{2} \varpi^{\alpha_{2}}, \quad v^{\prime}=\frac{1}{2} \varpi^{2 p}+u,
$$

so verschwinden auf der linken Seite alle Glieder für

$$
h=2^{p-5}+1, \ldots, 2^{p-4}+2^{p-5} \text {; }
$$


auf der rechten Seite alle Glieder für

und man erhält:

$$
j=2^{p-5}+1, \ldots, 2^{p-3}
$$

$$
\begin{aligned}
& \text { (XIV) } \\
& 0==\sum_{k=1}^{h=2 y-5}(-1)^{\Sigma n^{\lambda_{h}} m^{\lambda_{h}}} \vartheta_{l \lambda_{h}} \vartheta_{l \lambda_{h} \operatorname{cog}_{p}-2 \alpha_{2} p+1} \\
& \text { - } \vartheta_{l \lambda_{h}}(u) \vartheta_{l \lambda_{h} \alpha q p-2 \alpha_{2 p+1}}(u)
\end{aligned}
$$

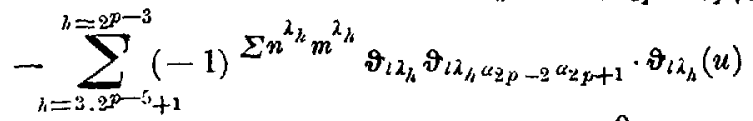

$$
\begin{aligned}
& \vartheta_{l \lambda_{1} \alpha_{2 p}-2 \alpha_{2 p+1}}(u)
\end{aligned}
$$

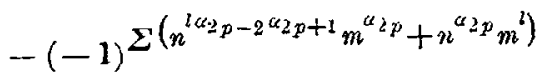

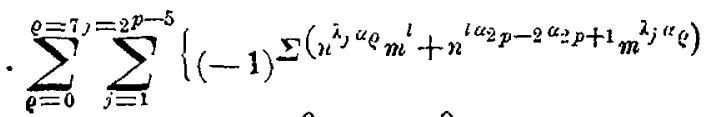

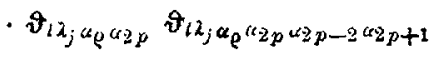

$$
\begin{aligned}
& \text { - } \vartheta_{l \lambda_{j} \alpha_{\rho} \alpha_{2 j}}\left(u j \vartheta_{l \lambda_{j} \alpha_{\rho} \alpha_{z} \alpha_{2} \alpha_{2-2} \alpha_{z} p+1}(u)\right\} \text {, }
\end{aligned}
$$

also eine Bezichung zwischen $10 \cdot 2^{p-5}$ Producten der Form $\vartheta_{\varepsilon}\{u\} \vartheta_{\varepsilon^{\prime}}(u)$. Insbesondere wird aus (XIV), für

$$
\begin{aligned}
& u=\frac{1}{2} \pi^{\lambda_{k} \alpha_{0} \alpha_{7} \alpha_{2 p} \alpha_{2 p-1}}, \quad\left(k=1,2, \cdots, 2^{p-5}\right):
\end{aligned}
$$

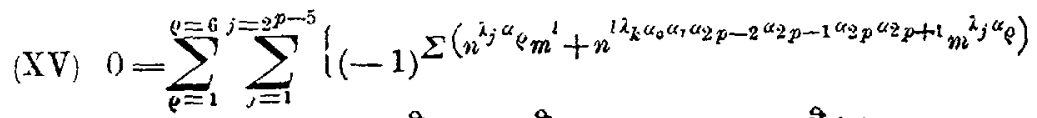

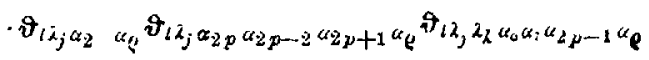

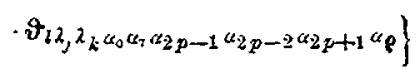

$$
\begin{aligned}
& \text { (wo } k=1,2, \cdots, 2^{p-5} \text { sein darf) }
\end{aligned}
$$

eine Beziehung zwischen $6 \cdot 2^{p-5}$ Producten, für die 0 - Werthe der Argumente.

4) $p \geqq 6$.

Setzt man in (XIV):

$$
u=\frac{1}{2} \pi^{\lambda_{k} 2 p-4^{\alpha_{2 p}}}, \quad\left(k=1,2, \cdots, 2^{p-\sigma}\right),
$$

so verschwinden auf der linken Seite die Glieder für $h=1,2, \cdots, 2^{p-6}$ und für $l_{i}=7 \cdot 2^{p-6}+1, \cdots, 2^{p-3}$; auf der rechten Seite alle Glieder für $j=2^{p-6}+1, \cdots, 2^{p-5}$, und man erhält: 


$$
\begin{aligned}
& (\mathrm{XVI}) 0=\sum_{h=2^{p-6+1}}^{h=2^{p-5}}(-1)^{\Sigma n^{\lambda_{h} \lambda_{k} \alpha_{2 p-4} \alpha_{2 p}} m^{\lambda_{h}}} \vartheta_{l \lambda_{h}} \vartheta_{l \lambda_{h} \alpha_{2 p-2} \alpha_{2 p+1}} \vartheta_{l \lambda_{h}} \lambda_{k} \alpha_{2 p-4} \alpha_{2 p}
\end{aligned}
$$

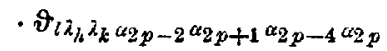

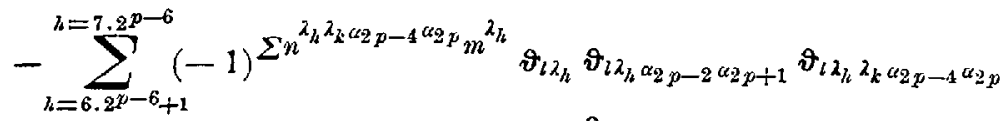

$$
\begin{aligned}
& \text { - } \boldsymbol{\vartheta}_{l \lambda_{h} \lambda_{k}} \alpha_{2 p-2} \alpha_{2 p+1} \alpha_{2 p-4} \alpha_{z p} \\
& -(-1)^{\Sigma\left(n^{l l_{k} \alpha_{z} p-4 \alpha_{2} \alpha_{m}} m^{\alpha_{2} p}+n^{\alpha_{2} p} m\right)}
\end{aligned}
$$

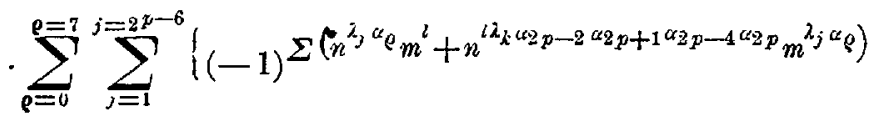

$$
\begin{aligned}
& \text { - } \left.\vartheta_{l \lambda_{j} \alpha_{2 j} \alpha_{\ell}} \vartheta_{l \lambda_{j} z_{p} \alpha_{2 p-2} \alpha_{2 y+1} \alpha_{\ell}} \vartheta_{l \lambda_{j} \lambda_{k} \alpha_{2 p-4} \alpha_{\ell}} \vartheta_{l \lambda_{j} \lambda_{k} \alpha_{2 p-2} \alpha_{2 p+1} \alpha_{2 p-4} \alpha_{\ell}}\right\} \text {; }
\end{aligned}
$$

für $k=1,2, \cdots, 2^{p-6}$; eine Beziehung zwischen $10 \cdot 2^{p-6}$ Producten, für die Nullwerthe der Argumente.

\section{$\S 18$.}

\section{Die Thetarelationen.}

Nach 1), 2), 3), 4), § 17, existiren im Ganzen folgende lineare bomogene Relationen:

a) Relationen zwischen Producten der Form $\vartheta_{\imath}(u) \cdot \vartheta_{\vec{z}}(u+w)$, mit Coefficienten der Form $\vartheta_{\varepsilon} \vartheta_{z}(w)$ :

1. für $p \geqq 3$ zwischen $6 \cdot 2^{p-3}$ solchen Producten (Formel (VI)),
2.,$p \geq 4$
$10 \cdot 2^{p-4} \quad$
(XIII))

b) Relationen zwischen Producten der Form $\vartheta_{\varepsilon}(u) \vartheta_{e^{\prime}}(u)$, wo $\varepsilon^{\prime}$ von $\varepsilon$ verschieden ist, mit Coefficienten der Form $\hat{\vartheta}_{\boldsymbol{\varepsilon}} \vartheta_{\mathbf{z}^{\prime}}$ :

1. für $p \geqq 3$ zwischen $4 \cdot 2^{p-3}$ solchen Producten (Formel (VIII)),
2.,$p \geqq 4$,
$6 \cdot 2^{p-1} \equiv$
(XI) oder $\left(\mathrm{XI}^{\prime}\right)$,
3. $" p \geqq 5 \quad$ \# $10 \cdot 2^{k-5} \quad$,
$"(",(\mathrm{XIV}))$.

c) Relationen zwischen Producten der Form $\vartheta_{\varepsilon} \vartheta_{\varepsilon}, \vartheta_{r \varepsilon} \vartheta_{r \varepsilon}$, wo die vier Indices von einander verschieden sind, für die Nullwerthe der Argumente, mit Zahlencoefficienten:

1. für $p \geqq 3$ zwischen $3 \cdot 2^{p-3}$ solchen Producten (Formel (X)),

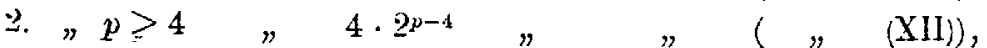

3. $n p \geq 5 \quad$ " $6 \cdot 2^{p-5} \quad$ " $\quad(" \quad$ (XV)),

4. $" p \geqq 6 \quad " 10 \cdot 2^{p-6} \quad, \quad " \quad(n \quad(\mathrm{XVI}))$. 
Zwischen diesen Relationen existirt eine grosse Anzahl von Beziehungen. Wir wollen hier nachweisen, dass sämmtliche Relationen der Arten 1. aus denen der Arten 2. folgen, wenn $p \geqq 4$; die 2. aus 3 . für $p \geqq \check{0}$ und die 3 . aus 4 . für $p \geqq 6$.

Um dies zunächst für die Relationen a) zu zeigen, machen wir auf Formel (XIII), § 17., eine Substitution: $\left\{\alpha_{3 p+1}, \alpha_{5}, \alpha_{6}, \alpha_{7}\right\}$. Hierdurch geht über: $(l)$ in $\left(l \alpha_{2 p+1} \alpha_{5} \alpha_{6} \alpha_{7}\right) ;\left(l \lambda_{\hbar}\right)$ in $\left(l \lambda_{k} \alpha_{2 p+1} \alpha_{5} \alpha_{6} \alpha_{7}\right)$ für $h=1,2, \cdots, 2^{p-4}$, aber in $\left(l \lambda_{h}\right)$ für $h=2^{p-1}+1, \cdots, 2^{p-s}$; also $\left[\lambda_{k}\right]$ im ersten Falle in $\left[\lambda_{k l}\right]$, im zweiten in $\left[\lambda_{k} \alpha_{2 p+1} \alpha_{5} \alpha_{6} \alpha_{7}\right]$; ebenso $\left(\alpha_{2 p}\right)$ in $\left(\alpha_{2 p} \alpha_{2 p+1} \alpha_{5} \alpha_{6} \alpha_{7}\right) ;\left(\alpha_{\rho}\right)$ in $\left(\alpha_{\rho} \alpha_{2 p+1} \alpha_{5} \alpha_{6} \alpha_{7}\right)$, wenn $\rho=0,1, \cdots, 4$, dagegen in $\left(\alpha_{\rho}\right)$ für $\varrho=5,6,7$. Daher wird (XIII), nach einfachen Reductionen, wenn man noch $\left(l \alpha_{2 p+1} \alpha_{5} \alpha_{0} \alpha_{7}\right)=(\beta)$ setzt:

$$
\begin{aligned}
& 0=(-1)^{\Sigma\left(n^{2} m^{\beta \alpha \alpha_{p}}+n^{\beta \alpha_{\nu p}} m^{2}\right)}
\end{aligned}
$$

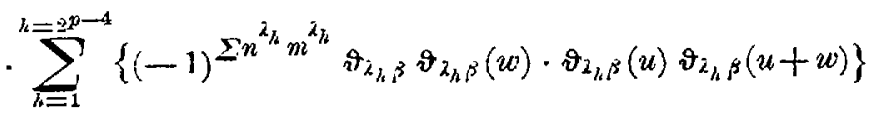

$$
\begin{aligned}
& +(-1)^{\Sigma\left(n^{l} m^{\alpha_{3 p}}+n_{\kappa}^{\alpha_{2} p} m^{b}\right)}
\end{aligned}
$$

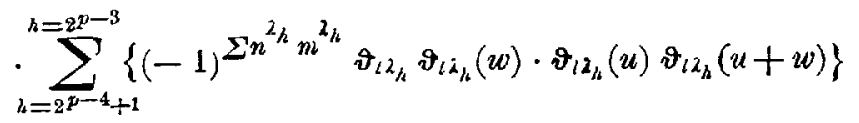

$$
\begin{aligned}
& -(-1)^{\Sigma n^{l \beta} m^{l j}} \sum_{\rho=0}^{\rho=4_{j}^{j}} \sum_{j=1}^{p-4}\left\{(-1)^{\Sigma\left(n^{2, \alpha_{\rho}} m^{l}+n^{l} m^{\lambda_{j} \alpha_{\rho}}\right)}\right.
\end{aligned}
$$

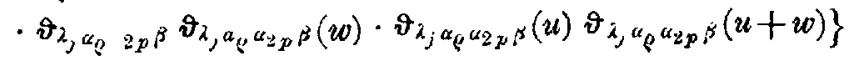

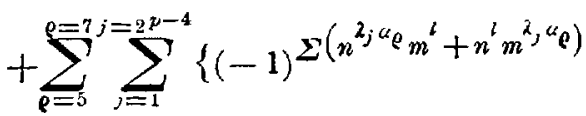

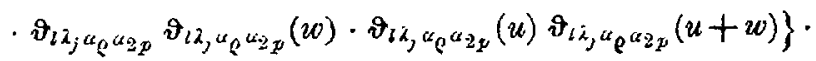

In dieser Formel stimmen aber 4 Summen mit 4 Summen, die in (XII) vorkommen, genau überein, und es folgt daher aus deren Verbindung:

$$
\begin{aligned}
& (-1)^{\Sigma n^{l \beta_{\mu l} l_{j}}} \cdot \sum_{\rho=0}^{e=4} \sum_{j=1}^{j=z^{p-4}}(-1)^{\Sigma\left(n^{2} \mu_{e} m^{l}+n^{l} m^{\left.\lambda_{j} \alpha_{\varphi}\right)}\right.}
\end{aligned}
$$

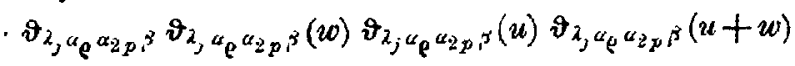

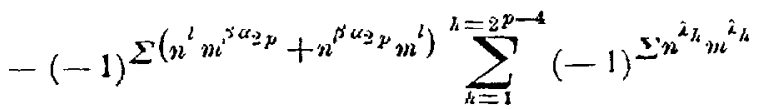

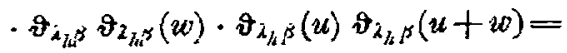




$$
\begin{aligned}
& =-\sum_{\rho=0}^{\rho=4} \sum_{j=1}^{j=2^{p-4}}(-1)^{\Sigma\left(n^{\lambda_{j} \alpha_{\rho}} m^{l}+n^{l} m^{\lambda_{j} \alpha_{e}}\right)} \\
& \text { - } \vartheta_{l \lambda_{j} \alpha_{p} \alpha_{2 p}} \vartheta_{l \lambda_{j} \alpha_{\ell} \alpha_{2 p}}(w) \cdot \vartheta_{l \lambda_{j} \alpha_{\ell} \alpha_{2 p}}(u) \vartheta_{l \lambda_{j} \alpha_{e} \alpha_{2 p}}(u+w)
\end{aligned}
$$

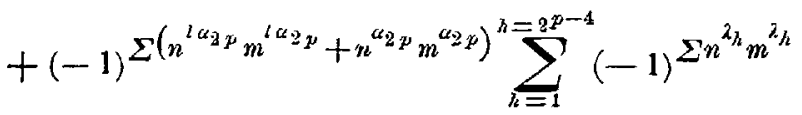

$$
\begin{aligned}
& \text { wo }(\beta)=\left(l \alpha_{2 p+1} \alpha_{5} \alpha_{6} \alpha_{7}\right) \text {. } \\
& \text { - } \vartheta_{l \lambda_{h}} \vartheta_{l \lambda_{h}}(w) \cdot \vartheta_{l \lambda_{h}}(u) \vartheta_{l \lambda_{h}}(u+w),
\end{aligned}
$$

Dies ist eine Formel der Art a), 1., abgeleitet aus a), 2. Die Formel würde in der That mit (VI) genau übereinstimmen, wenn man noch auf sie die Substitution $\left\{\alpha_{2 p} \alpha_{5} \alpha_{6} \alpha_{7}\right\}$ ausübte, wie eine einfache Rechnung zeigt.

Ganz analog ergeben sich die übrigen genannten Zusammenhänge. So bleibt, wenn man auf Formel (XI) eine Substitution $\left\{\alpha_{0} \alpha_{2 p+1}\right\}$ anwendet, die erste Summe $\sum_{h}$ unverändert, ebenso die Summe für $\varrho=0$; die übrigen 4 Summen, für $\varrho=1, \cdots, 4$, ändern sich aber, und es folgt daher, indem man die so erhaltene Gleichung von (XI) subtrahirt, eine Beziehung $z$ wischen $8 \cdot 2^{p-4}$ Producten der Form $\vartheta_{\varepsilon}(u) \vartheta_{\varepsilon^{\prime}}(u)$, die von der Art b), 1. wird und aus b), 2. abgeleitet ist. Die resultirende Fornel geht vermöge der Substitution $\left\{\alpha_{0} \alpha_{z_{p}}\right\}$ genau in (VIII) über.

Um b) 2. aus b) 3. abzuleiten, mache man auf (XIV) eine Substitution $\left\{\alpha_{0} \alpha_{7} \alpha_{2 p-2} \alpha_{2 p+1}\right\}$; dann ändern sich die beiden Summen $\sum_{h}$ nicht; die beiden Summen für $\varrho=0$ und $\varrho=7$ vertauschen sich nur untereinander, die 6 Summen $\sum_{j=1}^{\jmath=2^{p-5}}$, von $\rho=1, \cdots, 6$, ändern sich aber, und die so resultirende Gleichung, mit (XIV) verbunden, liefert eine Relation zwischen $12 \cdot 2^{p-5}$ Producten der Form $\vartheta_{\varepsilon}(u) \vartheta_{\varepsilon^{\prime}}(u)$, von der Art b),2. Dieselbe würde vermöge derSubstitution $\left\{\alpha_{0} \alpha_{7} \alpha_{z_{p-2}} \alpha_{2 p}\right\}$ in (XI') übergehen.

Um c), 1. aus c), 2. abzuleiten, mache man auf (XII) eine Substitution $\left\{\alpha_{0} \alpha_{4} \alpha_{5} \alpha_{2 p+1}\right\} ;$ um c), 2. aus c), 3. zu erbalten, auf (XV) eine Substitution $\left\{\alpha_{3} \alpha_{6} \alpha_{2 p-1} \alpha_{2 p+1}\right\}$; endlich um $\left.c\right)$, 3. aus c), 4. zu erhalten, auf (XVI) eine Substitution $\left\{\alpha_{0} \alpha_{7} \alpha_{2 p-3} \alpha_{2 p-1}\right\}$; und verbinde die resultirende Gleichung immer mit der ursprünglichen Gleichung. 
$\S 19$.

Die Thetarelationen für $p=5$.

Die in $\$ 8$. definirten , ausgezeichueten" Systeme ron $2 p+1$ Charakteristiken bestehen für $p=\mathbf{5}$ aus Systemen von 11 geraden Charakteristiken mit ungerader Summe, von der Art:

wo die

$$
\left(\alpha_{0}\right) \equiv\left(\alpha_{1}\right),\left(\alpha_{2}\right), \cdots,\left(\alpha_{11}\right),
$$

$$
\begin{aligned}
& \left(\alpha_{t}\right),\left(\alpha_{t} \alpha_{x} \alpha_{2} \alpha_{\mu} \alpha_{v}\right),\left(\alpha_{0} \alpha_{t} \alpha_{x}\right) \text { gerade, } \mid<\$ \$ * \$ \lambda \cdots, \\
& \left.\left(\alpha_{1} \alpha_{x} \alpha_{2}\right),\left(\alpha_{0} \alpha_{1} \alpha_{x} \alpha_{2} \alpha_{u}\right),\left(\alpha_{0}\right) \text { ungerade. }\right\} \text { von } 1,2, \ldots, 11 \text {. }
\end{aligned}
$$

Es ist sehr leicht, alle existirenden ausgezeichneten Systeme aus einem solchen abzuleiten und etwa alle von der Summe $\left(\alpha_{0}\right)$ der Form nach anzuschreiben, von den übrigen Summen je einen Repräsentanten. Indessen möge es hier genügen, diejenigen Systeme anzuschreiben, welche wir zu den folgenden Beweisen allein gebrauchen; nämlich alle, welche die 5 Charakteristiken

$$
\left(\alpha_{1}\right),\left(\alpha_{2}\right),\left(\alpha_{3}\right),\left(\alpha_{4}\right),\left(\alpha_{5}\right)
$$

enthalten. Dieselben kommen nach $\S 10$, 1., 2 ., und 5 , in

$$
\frac{11.10 \ldots 7}{1.2 \ldots 5} \cdot \frac{R_{2} R_{4} \ldots R_{10}}{1.2 .3 \ldots 11} \cdot \frac{1.2 .3 .4 .5}{R_{3} \cdot R_{8} \cdot R_{10}}=72
$$

ausgezeichneten Systemen vor, und zwar nur in den Systemen:

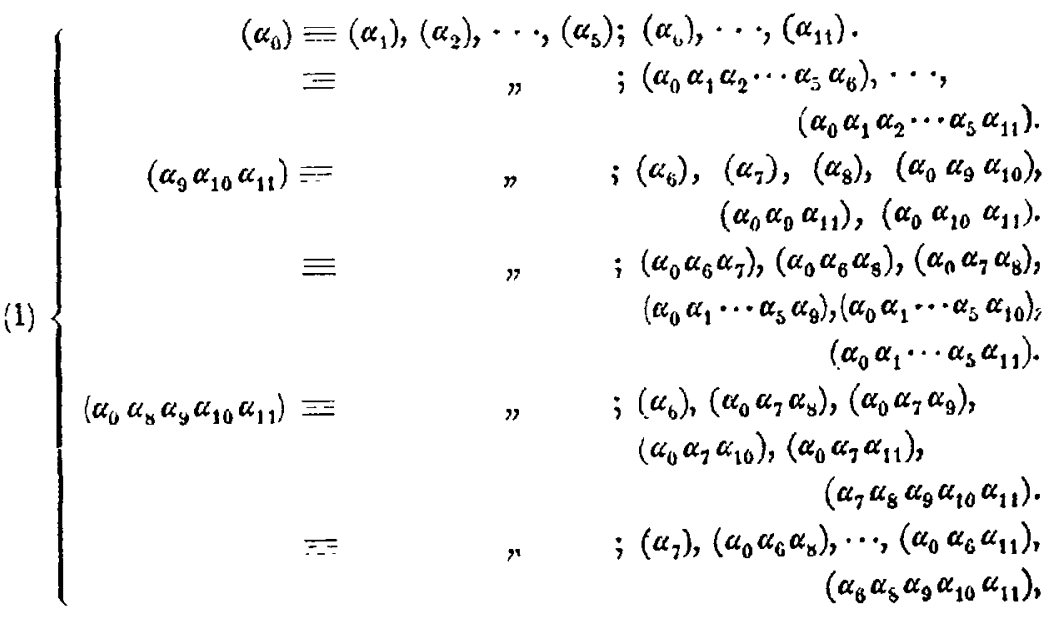

wo man noch die Indices $6,7, \cdots, 11$ auf alle Weisen zu vertauschen hat.

Um die Formeln dur $\$ \S 16$. und 17. anzuwenden, hat man dort zu setzen: 


$$
\begin{gathered}
l=\alpha_{0} \alpha_{8} \alpha_{10}, \quad l_{1}=\alpha_{8} \alpha_{9}, \quad l_{2}=\alpha_{10} \alpha_{11}, \\
\lambda_{1}=0, \quad \lambda_{2}=\alpha_{5} \alpha_{9}, \quad \lambda_{3}=\alpha_{10} \alpha_{11}, \quad \lambda_{1}=\alpha_{3} \alpha_{19} \alpha_{10} \alpha_{11} .
\end{gathered}
$$

Dann liefert zunächst Formel (XI'), \& 17., nach einer auf die Indices ausgeübten Substitution $\left\{\alpha_{0} \alpha_{v}\right\}$ eine Formel von der Gestalt:

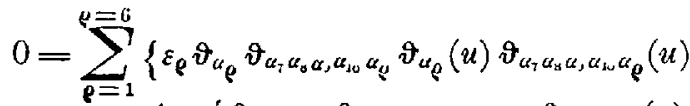

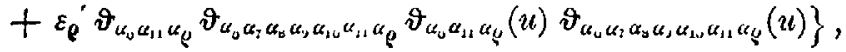

insbesondere für $u=\frac{1}{2} \widetilde{\omega}^{\omega_{j} \alpha_{c} \omega_{\tau} \alpha_{s}}$ eine Relation der Form:

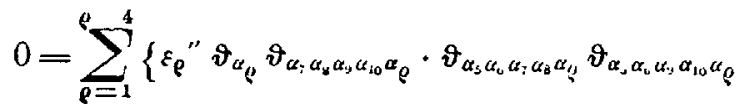

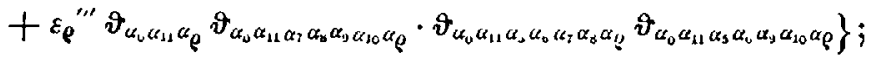

wo überall die $\varepsilon_{\rho}, \varepsilon_{\rho}{ }^{\prime} \cdots$ nur die Werthe \pm 1 haben.

Die formel (XIV), § 17, liefert ebenso eine Beziehung, wobei die Zahlencoefficienten am einfachsten durch Einsetzen von $u=\frac{1}{2} \widetilde{\Phi}^{\alpha_{1} a_{Q}}$ bestimmt werden:

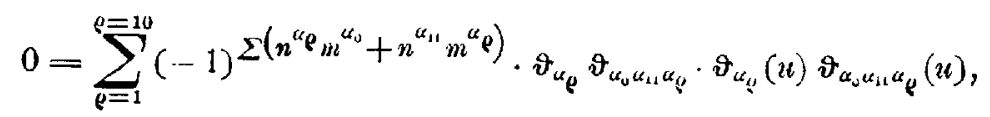

woraus, für $u=\frac{1}{2} \varpi^{\alpha_{\gamma} \alpha_{0} \alpha_{s} \alpha_{s 0}}$ :

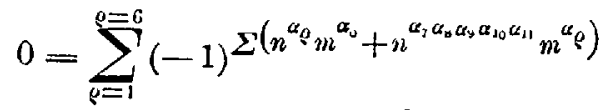

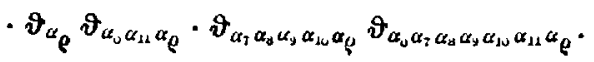

Die Relation (2) ist eine Folge der Relationen der Art (4); (3) soleher von der Art (5).

Wir stellen uns nun die Aufgabe, solche Systeme von geraden Thetafunctionen anzugeben, welche für die Nullwerthe der Argumente verschioinden können.

1. Sei zunächst:

$$
\begin{aligned}
& \boldsymbol{\vartheta}_{\alpha_{1}}=\vartheta_{\alpha_{2}}=\vartheta_{\alpha_{1}}=\vartheta_{\alpha_{4}}=0, \\
& \vartheta_{\omega_{0} \alpha_{11} \alpha_{2}}=\vartheta_{\alpha_{0} \alpha_{11} \alpha_{2}}=\vartheta_{\alpha_{0} \alpha_{11} \alpha_{2}}=\vartheta_{\alpha_{\alpha_{0} \alpha_{1} \alpha_{4}}}=0 \text {. }
\end{aligned}
$$

In Formel (3) kommen diese 8 Functionen auf die 8 Glieder dieses Ausdrucks vertheilt vor, und dieselbe ist erfüllt. Ferner ist jede Formel der Art (3), welche in ihren ersten 7 Gliedern irgend 7 der genannten Grössen enthält, erfüllt, weil in dem $8^{\text {ten }}$ Gliede immer die $\vartheta$-Funetion vorkommt, deren Index gleich der Summe der Indices der ersten 
7 Functionen ist. Das Verschwinden eines Systems solcher 8 9-Functionen, für die 0-Werthe der Argumente, stellt also nur 7 Bedingungen für die Moduln dar.

2. Sei

$$
\boldsymbol{\vartheta}_{\alpha_{1}}=\boldsymbol{\vartheta}_{\alpha_{2}}=\boldsymbol{\vartheta}_{\omega_{3}}=\boldsymbol{\vartheta}_{\alpha_{4}}=\boldsymbol{\vartheta}_{\alpha_{s}}=\boldsymbol{\vartheta}_{\alpha_{1} \alpha_{2} \alpha_{s} \alpha_{s} \alpha_{s}}=0 \text {. }
$$

Alsdann ist Formel (5) erfiillt. Und jede Formel dieser Art (5), welche in ihren ersten 5 Gliedern irgend 5 der angegebenen 6 Grössen enthält, ist erfüllt, weil in dem 6 , Gliede immer die 9 -Function vorkommt, deren Index gleich der Summe der Indices der ersten 5 Functionen ist. Das Verschwinden eines solchen Systems von $6 \vartheta$-Functionen, für die 0 -Werthe der Argumente, stellt daher nur 5 Bedingungen für die Thetamoduln dar. Von solchen Systemen giebt es (\$ 10., 2., 5.) an Zabl:

3. S'sei

$$
\begin{aligned}
& R_{3} \cdot R_{n} \cdot R_{30} \\
& 2.3 .4 \cdot i \cdot 6
\end{aligned}
$$

oline dass

$$
\boldsymbol{\vartheta}_{\alpha_{\mathrm{t}}}=\boldsymbol{\vartheta}_{\alpha_{\mathrm{z}}}=\boldsymbol{\vartheta}_{\omega_{\boldsymbol{t}_{\mathrm{s}}}}=\boldsymbol{\vartheta}_{\alpha_{\mathrm{t}}}=\boldsymbol{\vartheta}_{\boldsymbol{\alpha}_{\mathrm{s}}}=0 \text {, }
$$

$$
\vartheta_{\alpha_{1} \gamma_{s} \alpha_{3} \alpha_{s} \alpha_{s}}=0
$$

ist. Macht man auf Formel (5) alle Substitutionen, durch welche $\left(\alpha_{1}\right), \cdots,\left(\alpha_{5}\right)$ erbalten bleiben, also wie die Systeme (1) zeigen, die Substitutionen:

(6) $\left\{\alpha_{0} \alpha_{1} \alpha_{32} \alpha_{3} \alpha_{4} \alpha_{5}\right\},\left\{\alpha_{0} \alpha_{33} \alpha_{10} \alpha_{11}\right\},\left\{\alpha_{0} \alpha_{7} \alpha_{10} \alpha_{11}\right\} \cdot\left\{\alpha_{0} \alpha_{7} \alpha_{8} \alpha_{3}\right\}$, sowie die erste mit den beiden anderu verbonden, und macht man daun - noch alle Vertauschungen der Indices $6,7, \ldots, 11$, so erhält man alle Formeln der Art $(\tilde{\mathfrak{o}})$, in deren 5 ersten Gliedern $\boldsymbol{\vartheta}_{\alpha_{1}}, \hat{\vartheta}_{\alpha_{2}}, \ldots, \boldsymbol{\vartheta}_{\alpha_{s}}$ vertheilt auftreten. Daher müssen, wenn diese Grössen verschwinden, ohne dass $\vartheta_{\alpha_{s} \alpha_{\alpha} \alpha_{s} \alpha_{s} \alpha_{s}}=0$ sein soll, noch alle Producte verschwinden:

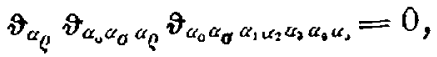

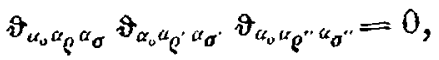

wo die Zahlen $\varrho, \sigma$, $\varrho^{\prime}$ etc. alle von einander verschieden sind und die Werthe $6,7, \ldots, 11$ annehmen sollen. Dazn ist erforderlich und hinreichend, dass ein System von folgender Art ebenfalls verschwindet:

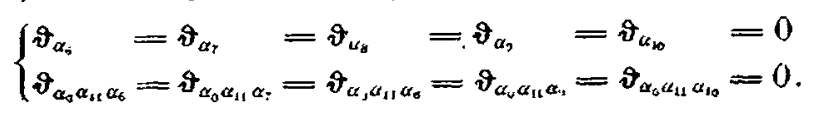

Vertauscht man alsdann, da auch $\vartheta_{\alpha_{6}}=0$ ist, $\tau$ mit 6 , (wo $\tau=1,2, \cdots 5$ ) und nimmt an, dass nicht ein System der Art

$$
\vartheta_{\alpha_{1} \alpha_{2} \alpha_{s} \alpha_{1} c_{j} \alpha_{v} \alpha_{\tau}}=0 \quad(\tau=1,2, \cdots 6) \text {, }
$$

d. h. ein System der Art 2. existirt, so sieht man, dass im Ganzen ein System der Art verschwinden muss: 


$$
\left\{\begin{array}{l}
\vartheta_{\alpha_{1}}=\vartheta_{\alpha_{2}}=\cdots=\vartheta_{\alpha_{10}}=0, \\
\vartheta_{u_{0} \alpha_{11} \alpha_{1}}=\vartheta_{u_{\alpha_{0} \alpha_{11} \alpha_{3}}}=\cdots=\vartheta_{\alpha_{v} \alpha_{1} \mu_{1}}=0,
\end{array}\right.
$$

Das Verschwinden solcher 20 Thetafunctionen zieht auch nicht das von weiteren nach sich. Denn wenn die $\vartheta_{\alpha_{1}}, \ldots, \vartheta_{\alpha_{s}}$ in den ersten 5 Gliedern von (5) vertheilt vorkommen, tritt eine der Grössen (7) im letzten Gliede auf. Nimmt man nun statt der ersten $5 \vartheta_{\alpha_{Q}}$ irgend 5 andere aus (8), von denselben Beziehungen, also statt

$$
\left(\alpha_{1}\right),\left(\alpha_{2}\right),\left(\alpha_{3}\right),\left(\alpha_{4}\right),\left(\alpha_{5}\right)
$$

Systeme derselben Form, in welcher nur einige der $\left(\alpha_{Q}\right)$ durch $\left(\alpha_{0} \alpha_{11} \alpha_{p}\right)$ ersetzt sind, d. h. macht man Substitutionen der Art:

$$
\left\{\alpha_{\rho} \alpha_{\sigma}\right\},\left\{\alpha_{0} \alpha_{11}\right\},\left\{\alpha_{0} \alpha_{11} \alpha_{\rho} \alpha_{\sigma}\right\},(\rho \gtrless \sigma \text {, von } 1,2, \ldots, 10)
$$

so gebeu die Grössen (8) nur in einander über. Ebenso sind, wie in 1. gezeigt, die Relationen (3) durch (8) befriedigt.

Die 20 Indices des Systems (8) sind in zwei Reihen von je 10 geschrieben:

$$
\begin{aligned}
& \left(\alpha_{1}\right), \quad\left(\alpha_{2}\right), \quad \cdots,\left(\alpha_{10}\right) ; \\
& \left(\alpha_{0} \alpha_{11} \alpha_{1}\right),\left(\alpha_{0} \alpha_{11} \alpha_{2}\right), \cdots,\left(\alpha_{0} \alpha_{11} \alpha_{10}\right),
\end{aligned}
$$

und diese beiden Reihen sind einer ungeraden Charakteristik, $\left(a_{n}\right)$, zugeordnet, indem rämlich jede Reilue aus 10 der 11 Charakteristiken eines "ausgezeichneten "Systems mit der Summe $\left(\alpha_{0}\right)$ besteht. Indessen ist die Gesammtheit der 20 Charakteristiken nicht $\left(\alpha_{0}\right)$ allein zugeordnet; sondern bei veränderter Vertheilung der 20 in 2.10 im Ganzen 256 verschiedenen ungeraden Charakteristiken, nämlich:

(9) $\left(\alpha_{0}\right),\left(\alpha_{11} \alpha_{\ell} \alpha_{\sigma}\right),\left(\alpha_{0} \alpha_{\rho} \alpha_{\sigma} \alpha_{\tau} \alpha_{\zeta}\right),(\rho \gtrless \sigma \gtrless \tau \gtrless \xi$, von $1,2, \ldots, j 0)$ die aus $\left(\alpha_{0}\right)$ durch die Substitutionen $\left(\sigma^{\prime}\right)$ hervorgehen.

Hieraus und aus der Anzahl der ausgezeichneten Systeme folgt auch, dass es auf

$$
\frac{1}{2^{9}} \cdot \frac{R_{2} R_{8} R_{6}}{1 \cdot 2 \cdot 3 \cdot 10} \frac{R_{8} R_{10}}{1 \cdot 10}
$$

verschiedene Arten möglich ist, Systeme von 20 Charakteristiken der Art (8) zu bestimmen. Sind von einem solchen System 5 Charakteristiken der Forn $\left(\alpha_{1}\right),\left(a_{2}\right), \cdots,\left(\alpha_{5}\right)$ gegeben, so sind die 15 übrigen, wie wieder (1) lehrt, auf 27 verschiedene Weisen wählbar.

Das System (8) ist wieder 5 Bedingungen äquivalent.

4. Sei endlich das System (8) gegeben und ausserdem

$$
\vartheta_{a_{12}}=0
$$

Dann miissen nach $(5)$ und $\left(6^{\prime}\right)$ noch alle Produete der Form

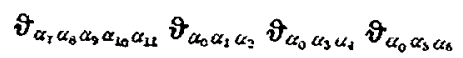


mit allen Vertauschungen aller Zahlen $1,2, \ldots, 11$ verschwinden. Entweder hat man also

$$
\vartheta_{\alpha_{14}}=0, \quad \vartheta_{\alpha_{0} \alpha_{2} \alpha_{2}}=0
$$

oder

$$
\vartheta_{\omega_{11}}=0, \quad \vartheta_{\omega_{i} \alpha_{8} \omega_{y} \alpha_{\alpha_{v} \alpha_{12}}}=0 .
$$

Dies Letztere führt aber zu einem System von derselben Art, als wenn man

$$
\vartheta_{u_{v} \alpha_{i} u_{d}}=0, \quad \vartheta_{\alpha_{u} \alpha_{,}, u_{10}}=0
$$

setzt; denn die Substitution $\left\{\alpha_{0} \alpha_{9} \alpha_{10} \alpha_{11}\right\}$, welche (8) unverändert lässt, fübrt beide in einander über. Daher kann man (13) statt (12) annehmen. Neben (11) kann man dann weiter annehmen, dass $\vartheta_{\alpha_{1} \alpha_{2} \alpha_{2} \alpha_{3} \alpha_{11}}$ uicht $=0$, da die Substitution $\left\{\alpha_{0} \alpha_{1} \alpha_{2} \alpha_{11}\right\}$ statt dessen liefert:

$$
\boldsymbol{\vartheta}_{\omega_{0} \alpha_{1} \alpha_{2}}=0, \quad \boldsymbol{\vartheta}_{\alpha_{11}}=0, \quad \boldsymbol{\vartheta}_{\alpha_{\mathrm{v}} \alpha_{2} \alpha_{\mathrm{s}}}=0
$$

und hierdurch ist auch schon der f'all (13), wenu man darin 7, 8,9, 10 mit 1, 2, 3, 4 vertauscht, weiter behandelt. Ferner zeigt (10), dass auch noch ein Ausdruck der Form

$$
\vartheta_{\alpha_{0} \alpha_{1} \alpha_{s}}=0
$$

werden muss; etc. Indem man so fortfährt, erkennt man, dass man zu einem System der folgenden Art kommt:

$$
\vartheta_{\alpha_{\rho}}=0, \quad \vartheta_{\alpha_{u} \alpha_{\rho} \alpha_{\sigma}}=0, \quad(\varrho \gtrless \sigma \text { von } 1,2, \ldots, 11) .
$$

Umgekehrt kaun nun wieder zeigen, dass das Verschwinden dieser 66 Thetafunctionen auch nicht das von weiteren geraden 'I'hetafunctionen nach sich zieht. Zu dem 'Zwecke bilde man aus den 66 auf alle mögliche Weisen solche 5, welche in den 5 ersten Gliedern einer Formel (5) vertheilt vorkommen können, d. h. alle Systeme von 5 Charakteristiken aus den $\left(\alpha_{\rho}\right),\left(\alpha_{0} \alpha_{\rho} \alpha_{\sigma}\right)$, die an Stelle von $\left(\alpha_{1}\right), \cdots\left(\alpha_{5}\right)$ treten können. Dies sind einmal die schon in 3) untersuchten, und es ist dort gezeigt, dass in dem letzten Giliede der Formel (5) dann immer eine Grösse aus (15) vorkommt. Sodann diejenigen, welche aus diesen durch die Substitutionen

$$
\left\{\alpha_{e} \alpha_{\sigma}\right\}, \quad\left\{\alpha_{0} \alpha_{e}\right\},
$$

hervorgehen; durch diese Substitutionen geht aber das System (15) nur in sich über. Endlich die Systeme der Art:

$$
\left(\alpha_{1}\right),\left(\alpha_{2}\right),\left(\alpha_{0} \alpha_{1} \alpha_{2}\right),\left(\alpha_{0} \alpha_{3} \alpha_{4}\right),\left(\alpha_{0} \alpha_{3} \alpha_{5}\right),
$$

mit den durch die obigen Substitutionen daraus folgenden; aber in diesem Falle ist die Summe der 5 Charakteristiken von der Form 
$\left(\alpha_{0} \alpha_{Q} \alpha_{a}\right)$ oder $\left(\alpha_{0}\right)$, und das entsprechende $\vartheta$ tritt im $6^{\text {ten }}$ Gliede der Formel (j) auf und ist zugleich in (10) enthalten.

- Die 66 zum System (10) gehörigen Charakteristiken

$$
\left(\alpha_{0}\right) ;\left(\alpha_{0} \alpha_{e} \alpha_{\sigma}\right), \quad(\varrho \gtrless \sigma, \text { von } 1,2, \cdots, 11)
$$

sivd einer ungeraden Charakteristik, $\left(\alpha_{0}\right)$, zugeordnet. In der That sind die aus je zweien der 66 Cbarakteristiken zu bildenden Gruppen alle vou den Furmen:

$$
\left[\alpha_{0} \alpha_{\rho}\right],\left[\alpha_{\rho} \alpha_{\sigma}\right],\left[\alpha_{0} \alpha_{\rho} \alpha_{\sigma} \alpha_{\tau}\right],\left[\alpha_{\rho} \alpha_{\sigma} \alpha_{\tau} \alpha_{\xi}\right] \text {, }
$$

und $\left(\alpha_{0}\right)$ ist in 495 dieser 561 Gruppen enthalten, in 66 derselben nicht; dagegen die übrigen ungeraden Charakteristiken sind je in 271 derselben enthalten, in 290 nicht. - Die Zuordnung zu . $\left(\alpha_{0}\right)$ kann auch mittelst der ",ausgezeichneten "Systeme von je 11 Charakteristiken geschehen; denn aus den Charalteristiken (15') lassen sich 12 solche Systeme bilden, welche alle $\left(\alpha_{0}\right)$ zur Summe haben:

$$
\left\{\begin{aligned}
\left(\alpha_{0}\right) & \equiv\left(\alpha_{1}\right),\left(\alpha_{2}\right), \cdots,\left(\alpha_{11}\right), \\
& \equiv\left(\alpha_{1}\right),\left(\alpha_{0} \alpha_{1} \alpha_{2}\right), \cdots,\left(\alpha_{0} \alpha_{1} \alpha_{11}\right), \\
& \equiv\left(\alpha_{2}\right),\left(\alpha_{0} \alpha_{2} \alpha_{1}\right), \cdots,\left(\alpha_{0} \alpha_{12} \alpha_{11}\right), \\
& \equiv \cdots,
\end{aligned}\right.
$$

und jedes einzelıe dieser Systeme fülırt auf gleiche Weise zu den 66 Charakteristiken (15') (vgl. \& 11., 2) 6)). Dagegen Jassen sich aus (15') nur je 4 ausgezeichnete Systeme bilden, welche eine von $\left(\alpha_{0}\right)$ verschiedene ungerade Clarakteristik zur Summe haben; und solche 4 Systeme (wie etwa

$$
\left(\alpha_{1} \alpha_{2} \alpha_{3}\right) \equiv\left(\alpha_{0} \alpha_{1} \alpha_{2}\right),\left(\alpha_{0} \alpha_{2} \alpha_{3}\right),\left(\alpha_{0} \alpha_{3} \alpha_{1}\right),\left(\alpha_{4}\right),\left(\alpha_{5}\right), \cdots,\left(\alpha_{11}\right),
$$

und die 3 durch die Substitutionen $\left\{\alpha_{0} \alpha_{1}\right\},\left\{\alpha_{0} \alpha_{2}\right\},\left\{\alpha_{10} \alpha_{3}\right\}$ hieraus hervorgehenden) führen auf ein System der Art $\left(15^{\prime}\right)$, das aber von diesem verschieden ist.

Aus dieser Zuordnung eines Systems (15') zu 12 ausgezeichneten Systemen (16) ergiebt sich zugleich, dass es auf

$$
\frac{L_{\mathrm{in}}}{12}=\frac{R_{2} R_{4} R_{\mathrm{f}} R_{8} R_{\mathrm{s}}}{1 \cdot 2 \cdot 3 \cdot 4 \cdots 12}
$$

verschiedene Weisen möglich ist, Systeme der Art. (15') oder (15) zu bestimmen. Sind die Gleichungen (8) gegeben, so kann man nach (9) das System der übrigeu verschwindenden $\vartheta$ noch auf $2^{s}$ verschiedene Weisen wählen. Ist ausser (8) noch $\vartheta_{u_{u}}=0$ gegeben, so liefert dies neben System (15) noch 45 weitere nicht $\left(\alpha_{0}\right)$ zugeordnete Systeme derselben Art, von denen irgend eines an Stelle von (15) gesetat werden kann. 
Das System (15) ist 6 Bedingungen äquivalent.

5. Es sollen jetzt noch die Relationen untersucht werden, welche vermöge (15) für die Differentialquotienten der Thetafunctionen, für die 0 -Werthe der Argumente, entstehen.

Wendet man auf Formel (14) dieses Paragraphen nach einander die Substitutionen an:

$$
\left\{\alpha_{1} \alpha_{2} \alpha_{3} \alpha_{1} \alpha_{10} \alpha_{11}\right\},\left\{\alpha_{1} \alpha_{2} \alpha_{3} \alpha_{1} \alpha_{3} \alpha_{9}\right\}, \quad\left\{\alpha_{10} \alpha_{11}\right\}
$$

nit audern Worten: ersetzt man das System

durch:

$$
\left(\alpha_{0}\right) \equiv\left(\alpha_{1}\right),\left(\alpha_{1}\right), \cdots,\left(\alpha_{11}\right)
$$

$$
\begin{aligned}
\left(\alpha_{0}\right) \equiv & \left(\alpha_{1} \alpha_{3} \alpha_{3} \alpha_{10} \alpha_{11}\right),\left(\alpha_{2} \alpha_{3} \alpha_{3} \alpha_{10} \alpha_{13}\right),\left(\alpha_{3} \alpha_{3} \alpha_{9} \alpha_{10} \alpha_{11}\right),\left(\alpha_{4} \alpha_{5} \alpha_{9} \alpha_{10} \alpha_{11}\right), \\
& \left(\alpha_{5}\right),\left(\alpha_{6}\right),\left(\alpha_{3}\right), \\
& \left(\alpha_{1} \alpha_{2} \alpha_{3} \alpha_{4} \alpha_{8}\right),\left(\alpha_{1} \alpha_{2} \alpha_{3} \alpha_{4} \alpha_{9}\right), \quad\left(\alpha_{1} \alpha_{2} \alpha_{3} \alpha_{4} \alpha_{10}\right), \quad\left(\alpha_{1} \alpha_{2} \alpha_{3} \alpha_{4} \alpha_{11}\right),
\end{aligned}
$$

so nimmt (4) die Gestalt an:

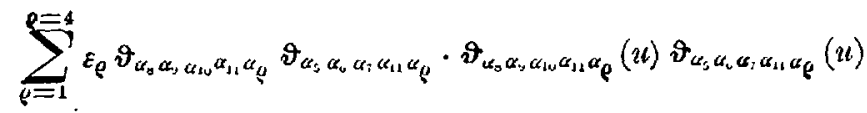

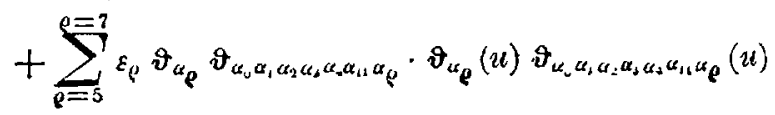

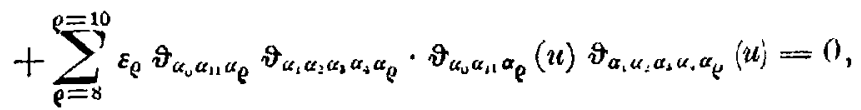

wo die $\varepsilon_{\rho}$ die Werthe \pm 1 haben.

Vermöge der Beziehungen (8) oder (15) reducirt sich diese Gleichung auf eine 4-gliedrige. Setzt man ausserdem

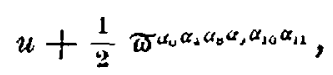

für $u$, so folgt dann nämlich:

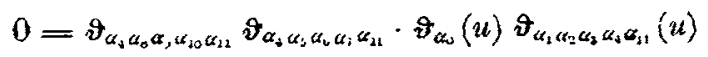

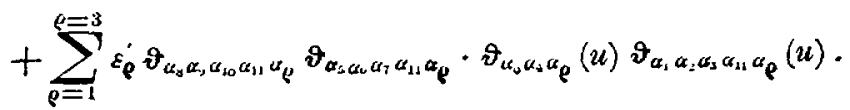

Da nun, wenn $(15)$ gilt, der factor von $\vartheta_{\alpha_{o}}(u)$ auch für die Nullargumente nicht verschwindet, dagegen

$$
\vartheta_{\alpha_{0} \alpha_{s} \alpha_{\rho}}=0, \quad \vartheta_{\alpha_{1} \alpha_{2} \alpha_{2} \alpha_{1} \alpha_{\varphi}}=0, \text { für } \varrho=1,2,3,
$$

so ergiebt die Differentiation der letzten Gleichung nach irgent einem der Argumente, mit Hülfe vou (15): 


$$
\left[\frac{\partial \vartheta_{\alpha_{o}}(u)}{\partial u_{a}}\right]_{0}=0
$$

für die Nollargumente.

Wenn also die Gleichungen (15) existiren, so verschwinden ausserdem noch für die Nullwerthe der Argumente die ersten partiellen Differentialquotienten derjenigen ungeraden Function $\vartheta_{\alpha_{0}}(u)$, deren Charakteristik $\left(\alpha_{0}\right)$ dem System der Charakteristiken der 66 Functionen von (15) zugeordnet ist. Das Bestehen eines Gleichungssystems der Art (15) und (17) tritt aber, wie bekannt, *) bei den hyperelliptischen Thetafuuctionen für $p=5$ auf.

*) Weierstrass ( 0 gl, die oben c. Abh. von Königsberger). Für die Gleichungen (17) insbesondere Riemann (vgl. die c. Abh. von Prym, \& 12.).

Erlangen, im November 1879. 